Portland State University

PDXScholar

$1-1-2012$

\title{
Investigation of Impedance Spectroscopy for Detection of Ovarian Cancer
}

\author{
Allison Mae Whited \\ Portland State University
}

Follow this and additional works at: https://pdxscholar.library.pdx.edu/open_access_etds Let us know how access to this document benefits you.

\section{Recommended Citation}

Whited, Allison Mae, "Investigation of Impedance Spectroscopy for Detection of Ovarian Cancer" (2012). Dissertations and Theses. Paper 68.

https://doi.org/10.15760/etd.68

This Dissertation is brought to you for free and open access. It has been accepted for inclusion in Dissertations and Theses by an authorized administrator of PDXScholar. Please contact us if we can make this document more accessible: pdxscholar@pdx.edu. 
Investigation of Impedance Spectroscopy for Detection of Ovarian Cancer

by

Allison Mae Whited

A dissertation submitted in partial fulfillment of the requirements for the degree of

Doctor of Philosophy

in

Applied Physics

Dissertation Committee:

Raj Solanki, Chair

Drake C. Mitchell

Jon Abramson

Shankar B. Rananavare

James E. Morris

Portland State University

2012 


\begin{abstract}
Electronic biosensors utilizing micron-scale interdigitate electrodes (IDEs) in an SD card format have been developed with the objective of fast, sensitive detection of ovarian cancer biomarkers CA-125, CEA, and He4. The signal generated by the biosensors is a result of electrochemical impedance spectroscopy (EIS), a technique which probes changes that occur in the biosensor's electrical properties when the biosensor has detected one of the target biomarkers. A label-free biosensor has been developed to detect CA-125 in spiked buffer at concentrations between 10 and 80 units $/ \mathrm{mL}$. A similar label-free biosensor was developed to detect CEA at concentrations between $10 \mu \mathrm{g} / \mathrm{mL}$ and $10 \mathrm{mg} / \mathrm{mL}$. A biosensor employing a protein-enzyme conjugated label was developed to detect $\mathrm{He} 4$ at concentrations ranging from 1.56 to $100 \mathrm{ng} / \mathrm{mL}$ in spiked buffer. All concentration ranges of CA-125, CEA, and He4 detected by the biosensors include the serum concentration currently used for clinical diagnosis of ovarian cancer. Efforts to improve the signals generated by the biosensors included altering the dimensions and composition of the IDEs used in the initial biosensors through software-created models. Modeled alterations included the size of the electrodes, the shape of the electrodes, and the incorporation of nanomaterials into the IDEs. An ideal geometry for the IDEs was developed through the models and IDEs with those dimensions were fabricated and tested against the IDEs used in the biosensors initially with the model-developed geometry improving the signal generated by the biosensor. Another attempt to
\end{abstract}


improve the biosensor's signal was to generate a single strand of DNA (sSDNA) that would bind to $\mathrm{CA}-125$, called an aptamer, that could be easily incorporated into the sensing layer on the IDEs. Through a multistep selection process nine different aptamers that exhibited binding to CA-125 were identified. 


\section{Dedication}

For Doug, Lola, Shep, Nanny, and Bea, the best support team a girl could ask for. 


\section{Acknowledgements}

This work would not have been possible without the help of a great many people including: Dr. Raj Solanki, Dr. Kanwar Vikas Singh, Dr. David R. Evans, Dr. Jianya Huan, Dr. Roberto Meza, and Dr. Arthur Vandenbark. 


\section{Table of Contents}

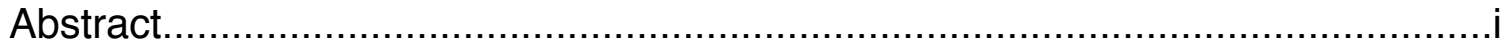

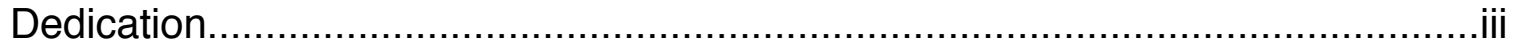

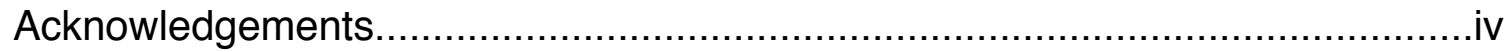

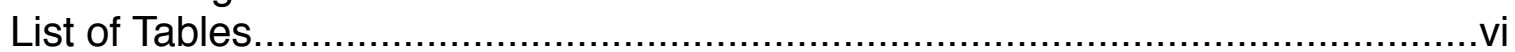

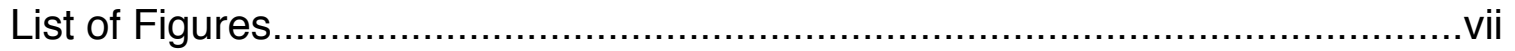

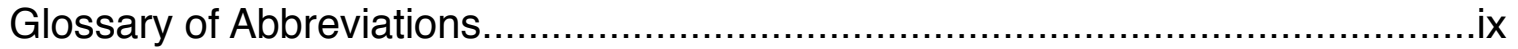

\section{Chapter 1}

Introduction to ovarian cancer, electrical biosensors, aptamers, and molecular biology

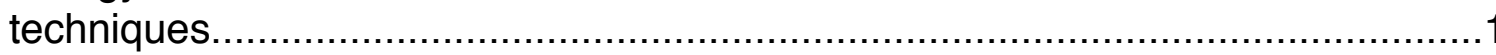

\section{Chapter 2}

IDEA chips and biosensor device design through computational models.

Chapter 3

Biosensor functionalization and immunosensor

development.

Chapter 4

Aptamer selection and molecular cloning procedures.

Chapter 5

Aptamer screening through sequence analysis and ELISA development.

Chapter 6

Results and

discussion.

Chapter 7

Conclusions and suggestions for future

work. 


\section{List of Tables}

Table 1. Summary of the five aptamer sequences chosen for synthesis. 131. Table 2. Summary of aptamers after further refinement through an additional 18 positive selection rounds and molecular cloning. 135.

Table 3. Summary of the sequences of the nine aptamers identified after the second round of molecular cloning. 137. 


\section{List of Figures}

Fig 1. Randles equivalent circuit for Nyquist plot obtained from electrochemical impedance spectroscopy. 9.

Fig 2. An example of a Nyquist plot obtained through EIS data. 11.

Fig 3. Fig 3. Summary of interactions occurring at electrode surface when binding is taking place between the sensing layer on the electrode and their alignment with the Randles circuit components. 12.

Fig 4. Rendering of aptamer-protein binding. 15.

Fig 5. Several views of the geometry of the IDEA chip. 20.

Fig 6. An example of the simulated electric field distribution surrounding the IDEA electrodes on the chip. 23.

Fig 7. The four positions on the IDEA chip model that were evaluated for the magnitude of the electric field and the current density. 24.

Fig 8. Comparison of current density distributions for four of the modeled gap distances. 25.

Fig 9. Graph of the decline in electric field and current density values with increasing gap distance. 26.

Fig 10. Graph of change in electric field values with changes in electrode height, electrode width, and gap distance. 28.

Fig 11. Results of first pitched geometry. 29.

Fig 12. Results of the second pitched geometry. 31.

Fig 13. The results of the nonconducting polyamide layer. 34 .

Fig 14. The results of a single layer of polyamide nanoparticles at a low density. 36.

Fig 15. Results of a high density single layer of polyamide nanoparticles. 38 .

Fig 16. Results of double layer of polyamide nanoparticles at low density. 40 .

Fig 17. Results of a high density double layer of polyamide nanoparticles. 42.

Fig 18. Comparison of effects from single and double layers of polyamide nanoparticles at low density. 43.

Fig 19. Comparison of effects from single and double layers of polyamide nanoparticles at high density. 44.

Fig 20. Comparison of high and low density polyamide nanoparticles. 45.

Fig 21. Determination of sensitivity of the devices fabricated through e-beam lithography. 47.

Fig 22. Overview of functionalization process. 51.

Fig 23. IDEA chip placed on a clean stage. 52.

Fig 24. Overview of functionalized multiplexed chip. 57.

Fig 25. Diagram representing electrodes functionalized for the detection of different targets. 58.

Fig 26. Diagram of the biolayer developed on the electrode of the He4 sensor. 62.

Fig 27. The double stranded PCR product from the modified SELEX procedure after being run through a denaturing polyacrylamide gel. 67 .

Fig 28. Overview of SELEX process. 69. 
Fig 29. Sequence diagrams of the aptamer libraries and primer used in PCR. 76. Fig 30. Results of PCR products created using different enzymes from different manufacturers visualized by ethidium bromide staining in a $10 \%$ polyacrylamide, $7 \mathrm{M}$ urea denaturing gel. 79 .

Fig 31. Gel composition determination. 81.

Fig 32. Cloning of PCR products. 84.

Fig 33. Map of the $\mathrm{pCR} \AA^{\circledR} 4-\mathrm{TOPO}{ }^{\circledR} .84$.

Fig 34. Verification of PCR insert size. 92.

Fig 35. Analysis of sequencing data using 4peaks software. 87.

Fig 36. Examples of the secondary structures of the aptamers obtained from mfold. 95.

Fig 37. Diagram of the ELISA for testing the aptamer pools. 99.

Fig 38. Overview of ELISA for synthesized aptamers. 101.

Fig 39. Detection of CA-125 in spiked buffer. 105.

Fig 40. CA-125 detection in 5\% rabbit serum. 107.

Fig 41. Detection of CEA in spiked buffer. 110.

Fig 42. CEA detection in $5 \%$ rabbit serum. 111.

Fig 43. Detection of He4 using faradaic EIS. 114.

Fig 44. Labeled detection of $\mathrm{He} 4$ in spiked buffer using biotinylated antibodies. 117.

Fig 45. Multiplexed detection of CA-125. 120.

Fig 46. Multiplexed detection of CEA in spiked buffer. 122.

Fig 47. Direct comparison of multiplexed detection in spiked buffer. 123.

Fig 48. Direct comparison of detection of CA-125 and CEA in $5 \%$ rabbit serum by the multiplexed chip. 126.

Fig 49. ELISA results from the initial aptamer pool after 16 modified SELEX rounds. 130.

Fig 50. ELISA results from 18 additional rounds of modified SELEX. 133.

Fig 51. Common structures among the 14 selected aptamers. 139. 


\section{Glossary of Abbreviations}

AFM: Atomic force microscopy

bp: Base pair

BSA: Bovine serum albumin

CEA: Carcinoembryonic antigen

DI: Deionized water

dNTPs: Deoxynucleotide triphosphates

dsDNA: Double-stranded DNA

EIS: Electrochemical impedance spectroscopy

ELISA: Enzyme linked immunoassay

FITC: Fluorescein isothiocyanate

HPLC: High-performance liquid chromotography

HRP: Horseradish peroxidase

HSA: Human serum albumin

IDE: Interdigitated electrode

IDEA: Interdigitated electrode array

IgG: Immunoglobulin G

LMP: Low melting point

nt: Nucleotide

PBS: Phosphate buffered saline

PBST: Phosphate buffered saline containing $0.05 \%$ Tween 20

PBSR: PBS containing a redox species 
PCR: Polymerase chain reaction

PDMS: Polydimethylsiloxane

RPM: Revolutions per minute

SAM: Self-assembled monolayer

SELEX: Systematic evolution of ligands by exponential enrichment

SEM: Scanning electron microscopy

ssDNA: Single-stranded DNA

TBE: Tris-borate-EDTA

TEMED: Tetramethylethylenediamine

TMB: Tetramethylbenzidine 


\section{Chapter 1:}

Introduction to ovarian cancer, electrical biosensors, aptamers, and molecular biology techniques 
1.10varian cancer. Ovarian cancer is the fifth-leading cause of cancer death in women [1]. Due to its nonspecific symptoms [2] and lack of an adequate physical exam, over 75 percent of cases are diagnosed in the late stages when the disease has metastasized beyond the primary site, leading to a five year survival rate of less than 30 percent [3]. By comparison, patients diagnosed in the early stages have a 90 percent survival rate over the same period [3].

An ovarian cancer diagnosis results in the classification of the disease into stage I, II, III, or IV, with the lower numbered stages being the earliest stages with the highest survival rates. In stages III and IV, the cancer has metastasized beyond the primary site. Unfortunately, a majority of diagnoses occur in these late stages when the survival rate is below 30\% [3]. There are three types of ovarian cancer: epithelial ( $90 \%$ of cases), germ cell carcinoma (5\% of cases), and stromal carcinoma (5\% of cases).

CA-125 and CEA are two biomarkers indicated in a number of cancers, including ovarian cancer $[4,5]$. Currently, CA-125 and CEA are used to determine the effectiveness of post-surgical treatment for ovarian cancer and colon cancer, respectively, and to monitor for disease recurrence [6]. The normal range for CA-125 in serum is less than 35 units $/ \mathrm{mL}(\mathrm{U} / \mathrm{mL})$. In healthy patients, CEA will not be present in serum at a greater concentration than $10 \mathrm{ng} / \mathrm{mL}$. While neither of these markers are suitable for early detection of ovarian cancer on their own, an elevated presence of both would appear to make early diagnosis of ovarian cancer more feasible. 
CA-125 is still a poorly understood protein. Despite being discovered in 1981, [7] its structure and function remain largely a mystery. It was only successfully cloned in 2001 [8] despite the ability of research groups and biological reagent suppliers to produce the antibodies to it for some time. It is a very large transmembrane protein weighing between 200 and $2000 \mathrm{kDa}$ with moieties that can separate from the larger protein. It is composed of a short cytoplasmic tail, a transmembrane domain, and a very large glycosylated extracellular domain all of which are poorly characterized.

The structure of CEA is better understood. It was discovered in 1965 [9]. With a molecular weight of about $200 \mathrm{kDa}$ it is, like CA-125, a heavily glycosylated protein with about $50 \%$ of its weight being composed of carbohydrate. It includes a small transmembrane domain and a large extracellular domain where the glycosylation is located.

Another promising biomarker is He4. It was discovered in 2003 and has been linked to the occurrence of ovarian cancer [10]. It is currently gaining ground in being utilized for ovarian cancer detection. It is found to be elevated in most patients at the early stage of the disease and it is indicative if the disease has spread beyond the primary site [10]. It is still too early to determine experimentally what the required range for detection should be. For the time being, populations of healthy and diseased women are monitored to determine what constitutes an elevated level of He4. The only assay currently on the market to detect $\mathrm{He} 4$ in serum samples is available from Fujirebio Diagnostics. 
According to their studies outlined in the product insert, $94.4 \%$ of healthy women had an He4 concentration of below 150 pM in serum [11] but there have been other estimates as high as $500 \mathrm{pM}$. Their studies included healthy women, women with benign gynecological disease, and women with various types of cancer including ovarian, endometrial, and gastrointestinal.

The structure of He4 is fairly well known. It weighs $13 \mathrm{kDa}$ but in its usual glycosylated state it weighs between $20-25 \mathrm{kDa}$ [12]. Thus it is a much smaller protein than CA-125 and CEA. He4 is part of a family of whey acidic fourdisulfide core (WFDC) proteins. Proteins classified as WFDC proteins are characterized by about 50 amino acid residues including eight conserved cysteine residues that form four disulfide bridges [13]. He4 is composed of one peptide and two of these WFDC regions [12].

1.2 Biosensor Overview. A survey of the literature shows that considerable effort is being directed towards developing biosensors that can overcome the limitation of the conventional bioassays currently in use to detect biological targets. These sensors target analytes including enzymes, cells, and antibodies or antigens.

Traditional bioassays are costly, time-consuming, and require specialized technicians and equipment to read them. A viable biosensor has to be quick, cheap, and portable. Whereas conventional assays are carried out with multiple step processes, typically consisting of several binding and washing steps, and an 
additional reading requirement, electronic biosensors incorporate the sensing element and the signal reader together on one device. The detection layer is developed on the transducer and when a recognition event occurs between the detection layer and the target, the environment around the transducer is altered, allowing a measurement [14].

The idea behind biosensors was developed in 1965 by Leland C. Clark, Jr., when he published a paper on an oxygen electrode he had developed [15]. His work on sensors to detect glucose relied on an enzymatic relationship between glucose and glucose oxidase [16]. His work on glucose detection resulted in the first commercially available biosensor in 1975. Glucose detection is a prime example of the need for biosensors. For people with diabetes, continuous testing of the level of glucose in their blood is required. A biosensor fits this need perfectly. It is patient friendly, rapid, and accurate. As long as it is provided with a constant stream of glucose oxidase, it is able to make measurements of glucose in biological samples like blood [14].

Environmental monitoring will benefit from the development of biosensors as well. Water sources can be checked for contaminants and air quality can be examined for pollutants. Food freshness and contamination can also be monitored with biosensors. Better detection of target molecules in both of these fields would lead to better safety and health for the population. 
Biosensors are typically broken down into one of four types: electrochemical, optical, mass, and thermal. All rely on different techniques to record the binding between the target and the sensing layer on the transducer.

Electrochemical biosensors are the largest class of biosensors [14]. They involve some kind of electron transfer reaction under certain physical constraints. Examples are electrochemical impedance spectroscopy (EIS), cyclic voltammetry $[17,18]$, and potential step chronoamperometry [19], and many more. In these sensors the transducer is generally an electrode or a system of electrodes. The biosensors developed in the research described in the following chapters fall into this category. There are several other types of biosensors that are summarized below.

- Optical biosensors utilize a fiber optic system as a transducer. They are divided into two categories: intrinsic, where the optical fiber undergoes direct interaction with the target, and extrinsic, where the optical fiber is used to translate the signal generated at the binding event into light [20]. The most well known example of an optical biosensor is surface plasmon resonance (SPR) [21].

- Mass biosensors rely on a piezoelectric frequency damping on the transducer's surface to convert the increase in mass at the detection layer, which comes about from the layer's binding with the target, into an informative signal [22-25]. A variation of this measurement of the change in micro -or nano-scale cantilever (MEMS and NEMS) vibration frequency. 
- Temperature-based biosensors exploit exothermic and endothermic reactions to generate a signal. In this case, a temperature sensor is used as a transducer.

- Many biosensors rely on the use of a label, or a molecule attached to the target, to either amplify or translate the signal provided by the transducer. Common choices for labels are enzymes, fluorescent molecules, and magnetic beads.

Techniques employing labels are non-ideal for a number of reasons including higher cost and extra processing steps. They can also alter the conformation of the molecule they are attached to, changing the binding ability, specificity, and kinematics of the system. While most of the sensors described in the following chapters rely on label-free detection there are a couple of exceptions.

Biosensors have found applications in a number of fields. The medical field stands to gain the most from the development of specialized biosensors. Ideally, a biosensor for any disease could be developed as long as the antigens and antibodies specific to it can be identified or a complimentary capture mechanism for the biomarker in question can be identified. Biosensors would also allow for disease detection in a point-of-care situation, benefiting patient care. Making them further ideal for disease detection, biosensors have the capacity to be multiplexed, allowing the device the ability to detect more than one disease marker at a time. This is a helpful feature as most diseases produce 
more than one protein marker. This multiplexing feature would in turn allow for highly specific disease detection so that chance of misdiagnosis is reduced.

1.3 Electrochemical Impedance Spectroscopy (EIS). In biosensors, binding of molecules at specific sites will result in the modification of the sensor's properties. More specifically, the formation of any kind of layer on the transducer of the biosensor, generally an electrode in EIS, affects the device's electrical characteristics. Additionally, any binding between the bioactive layer and its target causes the capacitance and resistance of the system to be altered.

EIS is an effective tool for investigating the electrochemical characteristics of modified transducers $[26,27]$. The impedance measurement is based on measuring the nonlinear response of the circuit elements to the changing input AC signal frequency. The impedance is measured with an impedance analyzer using the frequency response of the device. It can be expressed as the ratio of the system voltage phasor to the current phasor. Using phasors allows for incorporation of the phase angle between the current and voltage at different frequencies due to the nonlinear response.

To obtain EIS data, a small AC voltage, up to a several hundred $\mathrm{mV}$, and a frequency range from tens of $\mathrm{Hz}$ to several $\mathrm{MHz}$ is applied to the system and used to generate the signal. The impedance measurement data contains a real and an imaginary component. A Randles equivalent circuit can be fitted to the impedance data as a function of the frequency obtained through EIS 
measurement, as shown in Figure 1 [26-29]. Here, $R_{s}$ is the resistance of the bulk solution, $Z_{w}$ is the Warburg impedance that comes about as a result of the diffusion of ions in the solution between the electrodes. $C_{D L}$ is the double layer capacitance, and $\mathrm{R}_{\mathrm{ET}}$ is the electron transfer resistance.

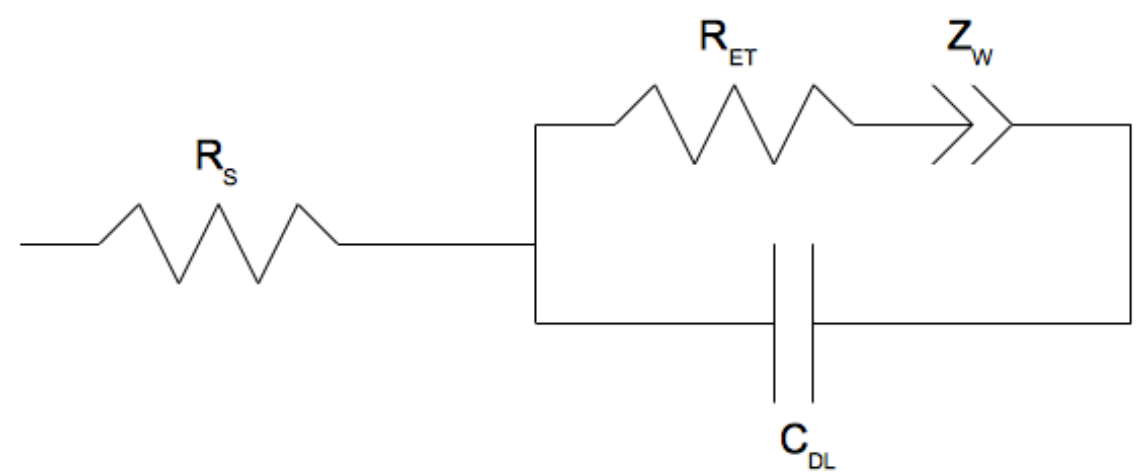

Fig 1. Randles equivalent circuit for Nyquist plot obtained from electrochemical impedance spectroscopy.

$R_{s}$ and $Z_{w}$ are affected solely by the bulk solution. As such, these values will not vary with electrical activity occurring at the solution/electrode interface. $C_{D L}$ and $R_{E T}$ on the other hand are dependent on the dielectric and the electron transfer resistance at the electrode interface, respectively. There are other circuit models that can be fitted to EIS data, but the Randles circuit is the most common choice and it is the one used during data analysis in the research described in the following chapters.

EIS has proven to be a sensitive method that can detect even minute changes in the capacitance and resistance of the transducer-bioactive layer interface $[30,31]$. The data can be collected in the presence of a redox probe. 
This measurement is called faradaic impedance spectroscopy and it is used to probe the biocatalytic reactions between the modified transducer and the captured target. The total electron transfer resistance, $R_{E T}$, of the system in this case is equal to the contributions from the electron transfer resistance due to the electrode itself, which is constant, and the electron transfer resistance from the redox probe, which is variable [32]. The $\mathrm{C}_{\mathrm{DL}}$, or double-layer capacitance, can be found the same way, by adding the inverses of the capacitive contributions from the electrode itself and its surface modifier and then taking the inverse of that.

EIS spectra can also be obtained without the use of a redox probe. This is called nonfaradaic impedance spectroscopy. In this case, the $C_{D L}$ values can be exclusively investigated without taking the other circuit elements into account $[32,33]$. The circuit elements that arise due to the presence of a redox probe, $R_{E T}$ and $Z_{w}$, can be assumed to be infinite, leaving a simplified circuit, with just the $R_{s}$ and $C_{D L}$ components in series. However, there needs to be no such assumption and values for $R_{E T}$ and $Z_{w}$ may be obtained using the circuit in Figure 1.

A common method to determine the values of the above four parameters of the system is to gather EIS data, plot the imaginary and real components of the impedance, and fit the resulting curve with circuit fitting software. One such example of this software is Z-view. The resulting curve is called a Nyquist plot as seen in Figure 2. Figure 3 provides an overview of the interactions occurring around the transducer and how those interactions align with the Randles circuit and Nyquist plot. 
Another method of using EIS data is to analyze the individual impedance measurements obtained at each frequency. The individual impedance values recorded under different conditions can be compared as long as they are at the same frequency. Frequencies between 50 and $5000 \mathrm{~Hz}$ produce the most linear signal for change in impedance under different conditions. An example of this type of data analysis would be to compare the change in impedance at a particular frequency for increasing concentrations of the molecule that is being detected and determining if there is a correlation.

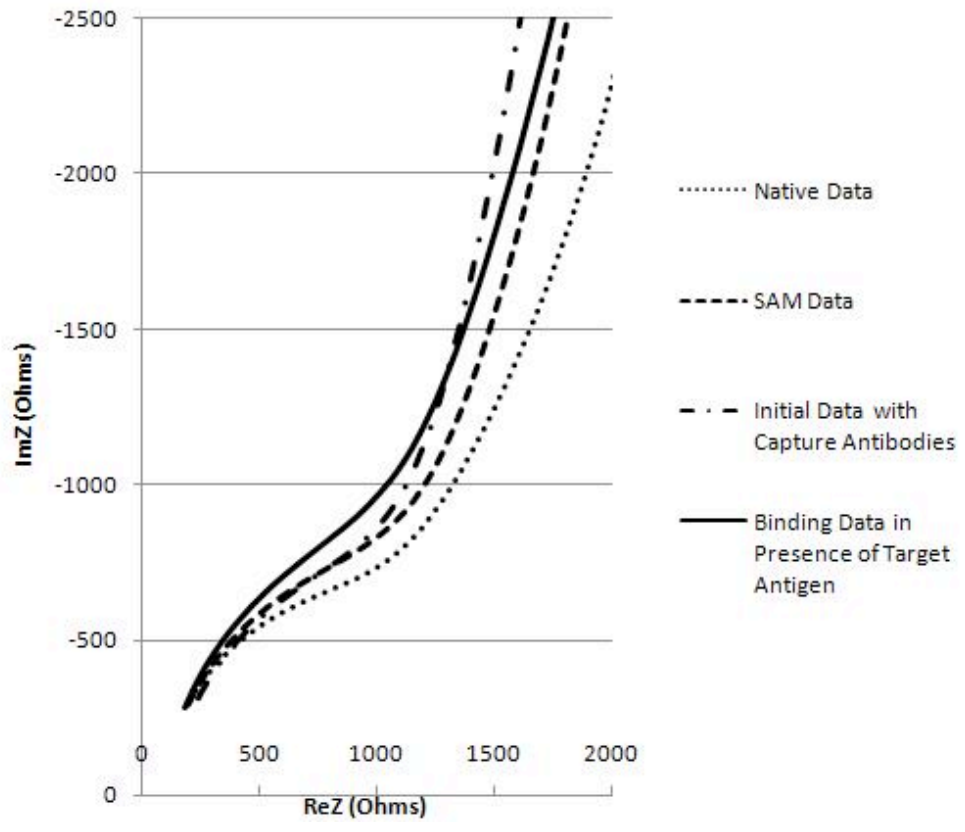

Fig 2. An example of a Nyquist plot obtained through EIS data. The semicircular portion of the graph is a result of the high frequency response of the system while the linear portion of the graph is generated from the low frequency response. The higher frequencies in the spectrum allow the fast events such as electron transport to be examined and the lower frequencies are a response of slower events such as mass transfer due to diffusion. 


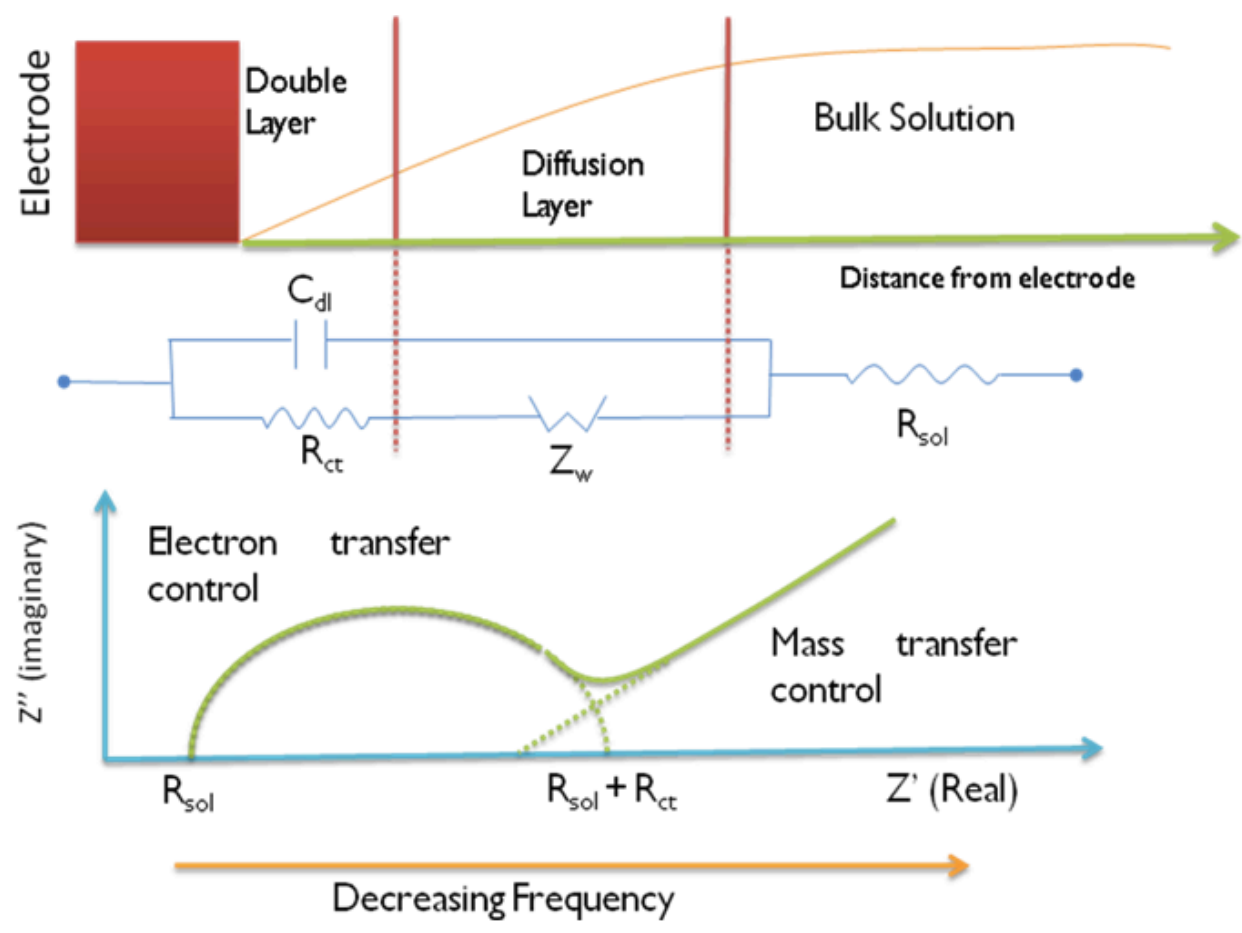

Fig 3. Summary of interactions occurring at electrode surface when binding is taking place between the sensing layer on the electrode and their alignment with the Randles circuit components. Immediately around the electrode's area is where the values for the $C_{D L}$ and $R_{E T}$ components are determined. In the diffusion layer there is still some ion flow and the $Z_{w}$ component dominates. Farthest out from the electrode is the bulk solution, Rs. The Nyquist plot summarizes which components of the Randles circuit can be determined from the plot.

1.4 EIS sensors in literature. The first impedimetric sensor was developed in 1986 [34] and the next followed in 1988 [35]. Many of these early sensors consisted of an insulator or polymer containing the probe molecule being layered on the interdigitated electrodes of the sensor. The resulting change in impedance was measured when the probe molecule's complementary molecule was detected by the sensing layer. The first immunosensor utilizing EIS as the detection method was reported in 1991 [36]. The biosensor was sensitive down to a concentration of $50 \mathrm{ng} / \mathrm{mL}$ of human antilgG. 
Self-assembled monolayer (SAM) modified electrodes were reported in 1997 [37]. Mirsky et al. monitored the impedance changes that occurred during probe molecule immobilization onto the SAM for different probe densities. The change in impedance was measured at $20 \mathrm{~Hz}$ where the change in impedance was purely capacitive. By making these measurements they were able to improve signal reproducibility by $10-30 \%$.

In 1996, Rickert et al. asserted that nonfaradaic EIS was preferable to faradaic detection. Though there was observed nonspecific binding and drift, the capacitive measurements reported using nonfaradaic EIS went down to the ng/ $\mathrm{mL}$ level [38].

In 2000, an early faradaic EIS sensor was reported on a SAM-modified gold electrodes [39]. In it several different routes to sensor functionalization were examined by comparing electron transfer resistance values. It was determined that it was possible to cover $97 \%$ of the electrode with an antibody sensing layer and that the addition of a redox probe allowed for detection to the $\mathrm{pg} / \mathrm{mL}$ level.

Recently there has been a push to create EIS biosensors capable of detecting more than one target at a time to increase the speed and ease of detection. A DNA-based sensor was recently developed to detect $\mathrm{Pb}^{2+}, \mathrm{Ag}^{+}$, and $\mathrm{Hg}^{2+}$ based on their interactions with DNA [40]. They were detected down to concentrations of $10 \mathrm{pM}, 10 \mathrm{nM}, 0.1 \mathrm{nM}$, respectively, in lake water and calf serum. 
DNA based sensors relying on hybridization between the target and the sensing layer have also been growing in popularity. Recently Hassen et al. reported the development of a DNA based sensor that implemented streptavidin coated magnetic nanoparticles and biotinylated DNA probes to detect HIV and HIVB DNA down to concentrations of $160 \mathrm{pmol}$ and $50 \mathrm{pmol}$ respectively [41].

RNA biosensors have also been developed. Park et al. have reported on a faradaic EIS sensor detecting a highly conserved region of the RNA genome of hepatitis C virus [42]. This particular RNA oligomer was detected down to a level of $26 \mathrm{pM}$.

A great deal of effort has also been put into developing EIS biosensors using novel materials. In 2011, a biosensor was reported that utilized electrodes modified with immolized E. coli specific DNA on cystine dendrites [43]. These electrodes, using EIS, were able to selectively detect E. coli over other waterborn pathogens over a range of concentrations from $10^{-14}$ to $10^{-6} \mathrm{M}$.

Novel films on electrodes are also drawing attention for their ability to be easily incorporated into the sensing layer of an EIS biosensor. One such film recently reported by Chen et al. contains polypyrrole, to promote conductivity and electrical activity, polypyrrolepropylic acid, to promote hydrophilicity at the same time providing covalent bond linkers, and gold nanoparticles, which promote stability, conductivity, and covalent bond linkers. This film was functionalized to detect human interleukin 5 in spikes phosphate buffered saline (PBS) and 1\% human serum [44]. The lowest detection level achieved was $10 \mathrm{fg} / \mathrm{mL}$ in PBS and 
$1 \mathrm{pg} / \mathrm{mL}$ in serum. In both samples, the dynamic range for detection was three orders of magnitude.

\subsection{Aptamers and their application in biosensors. Aptamers are single} strands of DNA or RNA that have a unique conformation that can be exploited to make them bind to protein targets other than their complementary strands [45, 46]. They have been used to bind to a large number of targets including aromatic ligands, amino acids, oligosaccharides, peptides, proteins, and cells [47, 48]. Figure 4 is a rendering of an aptamer bound to a protein [49].

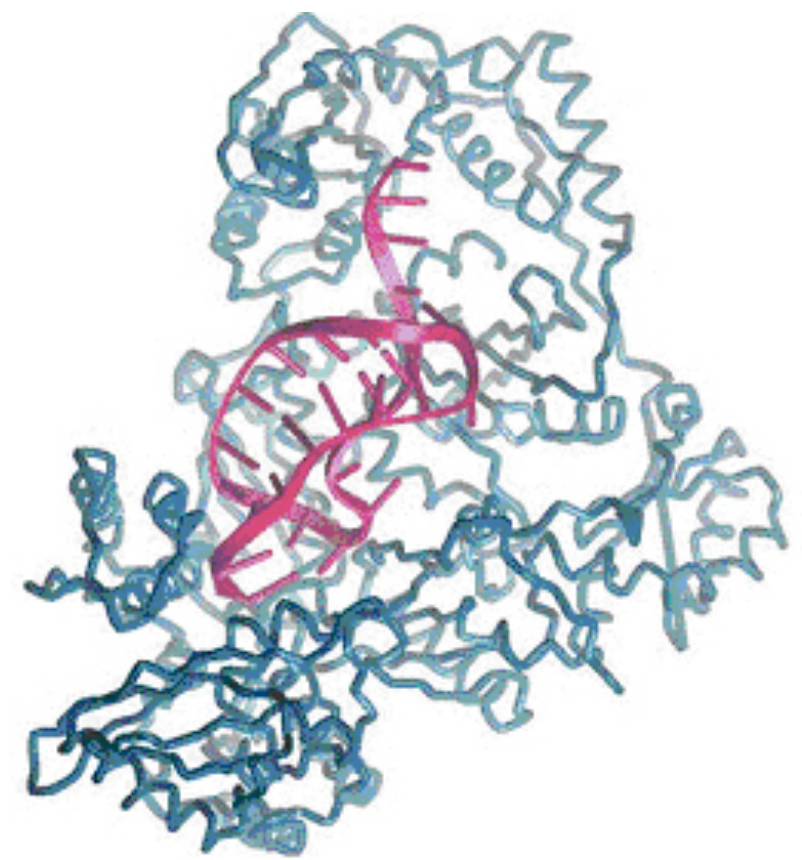

Fig 4. Rendering of aptamer-protein binding. The three dimensional structure of the aptamer enables it to bind with strong affinity for a particular region of the protein.

The identification of these binding sequences of the strands occurs though a selective process called SELEX, selective evolution of ligands by exponential 
enrichment $[45,46]$. The process begins with the selection of binding sequences or RNA or DNA from a library of random sequences of known length and end sequences. Through well-defined tertiary structure aptamers are able to bind to a target molecule. They exhibit dissociation constants in the picomolar and nanomolar range which is on the same order as some monoclonal antibodies [50].

Aptamers offer many advantages over traditional proteins. They can be synthesized cheaply and reliably unlike antibodies. Their overall structure can be evaluated, once selected for a target, yielding information about the structure of the target protein. They can also be modified chemically to make them more stable, to increase their shelf life, or to increase their binding affinity for the target. Their simple structure makes it easier to develop them into a sensing layer in a device. They are also more stable and less prone to denaturation than antibodies. The biggest obstacle to the development of aptamers is their reactivity with nucleases. This is primarily a problem with RNA aptamers. As such, we will be focusing on DNA aptamers. However, a slight structural modification to the ends of the aptamer makes it resistant to interaction with most nucleases found in biological samples [51].

1.6 Outline of dissertation. This dissertation will cover all procedures and results of the research in developing biosensors for the detection of ovarian cancer biomarkers. Chapter 2 contains background on the biosensor platform 
and the computational models created to improve its design. The results obtained from the models will be included along with information about the software used to generate the models. Chapter 3 covers the functionalization procedure for the biosensors as well as specific details about each of the antibody based biosensors that were developed for the detection of CA-125, CEA, and He4. Chapter 4 details the procedure used to create an aptamer pool for CA-125. Information about the molecular cloning process is also included. Chapter 5 deals with the methods and techniques used during the analysis of the aptamers obtained during the work done in Chapter 4. Work with sequence analysis and ELISAs is included. Chapter 6 contains the results obtained from the work detailed in Chapters 3-5. Discussion is included. Chapter 7 is a summary of the conclusions drawn from the results in Chapter 6 . There are also suggestions for future work. 


\section{Chapter 2:}

IDEA chips and biosensor device design through computational models 
2.1 IDEA Chip. The transducer is a key component of electrochemical biosensors. Optimized transducer design and electrolyte composition can lead to more sensitive and more effective biosensors. For this project one specific transducer based on past investigation design was used, but other configurations and variations on the current design were modeled as a way to see where the design could be improved.

Interdigitated electrode array (IDEA) chips were fabricated using standard photolithography techniques at SHARP Labs in Camas, WA. A 650 $\mu$ m thick silicon wafer was coated with a layer of silicon dioxide that was $1 \mu \mathrm{m}$ thick. A thin layer of gold was also deposited onto the surface. From there, microelectronic photolithography techniques and deep reactive ion etch (DRIE) were used to carry out the fabrication process.

Each IDEA chip consisted of eight electrode arrays, each with its own contact pad. The electrode arrays were made of gold, long known to be a biologically friendly material. Each array contained 39 individual electrodes measuring $1.5 \mu \mathrm{m}$ wide, $180 \mu \mathrm{m}$ long, and $0.2 \mu \mathrm{m}$ high. Electrode fingers were separated by a gap measuring $0.8 \mu \mathrm{m}$. The eight individual arrays were arranged such that they could be viewed as two distinct groups of four electrodes. The contact pads of the chip were designed in the secure digital (SD) card format. The individual geometries of the IDEAs were surveyed using SEM and AFM. The overall geometry of the chip can be seen in Figure 5. 

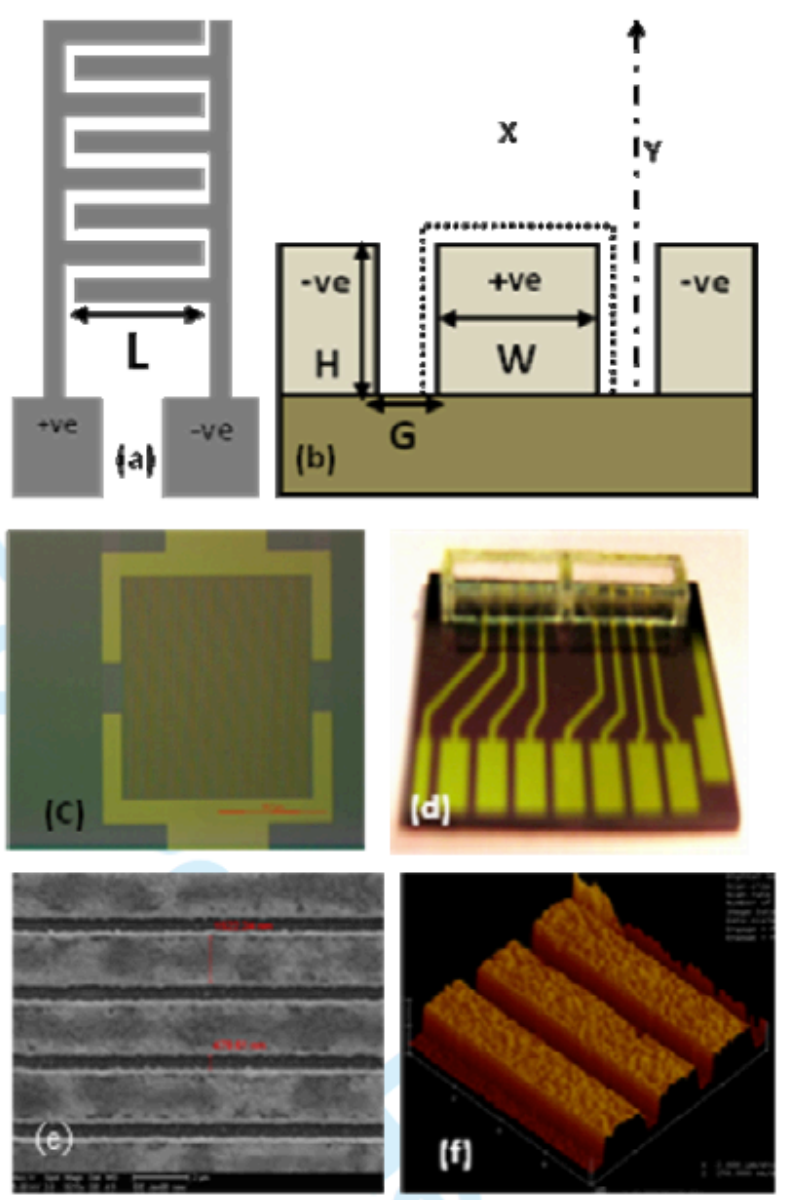

Fig 5. Several views of the geometry of the IDEA chip. (a) The interlocking electrode fingers each with a length of $180 \mu \mathrm{m}$, (b) Each electrode is $0.2 \mu \mathrm{m}$ high and there is a gap of $0.8 \mu \mathrm{m}$ between each of them, (c) the overview of each electrode array, (d) a comprehensive look at the chip with the eight IDEAs, (e) an SEM image of the electrodes and (f) an AFM image of the electrodes.

The IDEA chips were pre-cleaned in an oxygen plasma. Under certain circumstances, the chips may have been cleaned with a piranha solution, a mixture of 3:1 sulfuric acid and hydrogen peroxide which is commonly used as a cleaning solution in microelectronics. 
2.2 Computational modeling of the IDEA chip. Electric field and current density distribution on our IDEA chips were simulated using Ansoft Maxwell software. Maxwell utilizes finite element method analysis to solve electromagnetic problems for particular geometries. Though the program has 3D capabilities, it was decided for the purposes of this experiment that 2D analysis would provide sufficient information.

A geometric model identical to the actual IDEA chip was created in Maxwell. Phosphate buffered saline (PBS), a commonly used buffer in biological applications, was selected as the electrolyte, which has a relative permittivity of 80 and a bulk conductivity of 0.13 siemens $/ \mathrm{m}$. The AC input signal had an amplitude voltage of $100 \mathrm{mV}$ and zero degree phase angle with respect to the current and at a frequency of $60 \mathrm{~Hz}$. Both the background and excitation voltage parameters were consistent with the parameters used in the actual physical experiments. The device was assumed to be insulated from any background electromagnetic activity.

Maxwell software solves Maxwell's equations under certain conditions that are set in the model. Consider any point denoted by $\nabla \Phi(\mathrm{x}, \mathrm{y})$ that is describing the electric potential at each value of $x$ and $y$ where $\omega$ is the angular frequency at which the potential is oscillating. Then the conditions at this point can be described by

$$
\nabla \cdot(J+j \omega D)=0
$$


where $J$ is the current density and $D$ is the electric flux density. This equation can be modified to represent the conditions in terms of the electric field $E$ and the potential as shown below.

$$
\nabla \bullet[\sigma \boldsymbol{E}+j \omega \varepsilon \nabla \phi(x, y)]=0
$$

where $\sigma$ is the conductivity and $\varepsilon$ is the permittivity.

With each of the eight clusters of electrodes containing 39 individual interdigitated electrode fingers, some simplifications were made to the model. The cross sections of four individual but interlocking electrodes were simulated. In this way, the behavior of electrodes both on the outside edges and in the middle of the array were examined.

The following limitations were set for the problem solving component of Maxwell: $1 \%$ error, $100 \%$ refinement per pass with a maximum of ten passes and a minimum of one converged pass. Using these conditions, a graphical representation of the electric field and the current density were obtained. A sample of the representation of the electric field can be seen in Figure 6 .

Several variations to the existing design were explored. The effect of the gap width between the electrodes, both smaller and larger than the $0.8 \mu \mathrm{m}$ used in the current designs was examined.The electric field and current density that resulted from these modifications were evaluated. This includes overall distribution as well as evaluation at particular points on the electrodes, namely the edges and center, at 10 and $50 \mathrm{~nm}$ from the electrode surface. The positions 
are identified in Figure 7 . This allowed the strength of the electric field and current density of the resulting modifications to be compared as well as provided the values at different positions on the electrodes. In evaluating the field at varying distances from the electrode it is possible to evaluate the attenuation or augmentation of these parameters with distance.

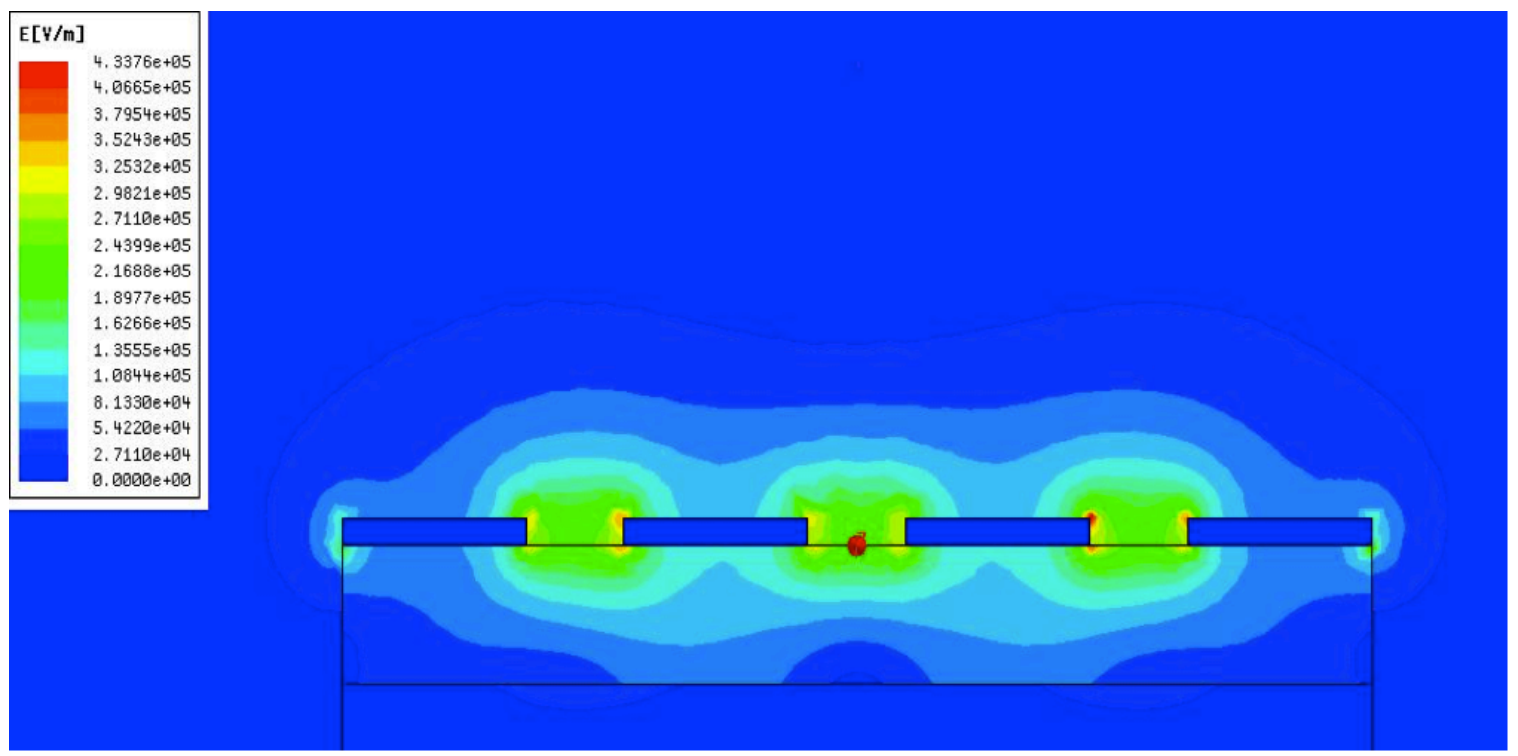

Fig 6. An example of the simulated electric field distribution surrounding the IDEA electrodes on the chip. This is the electric field for an unmodified electrode. 


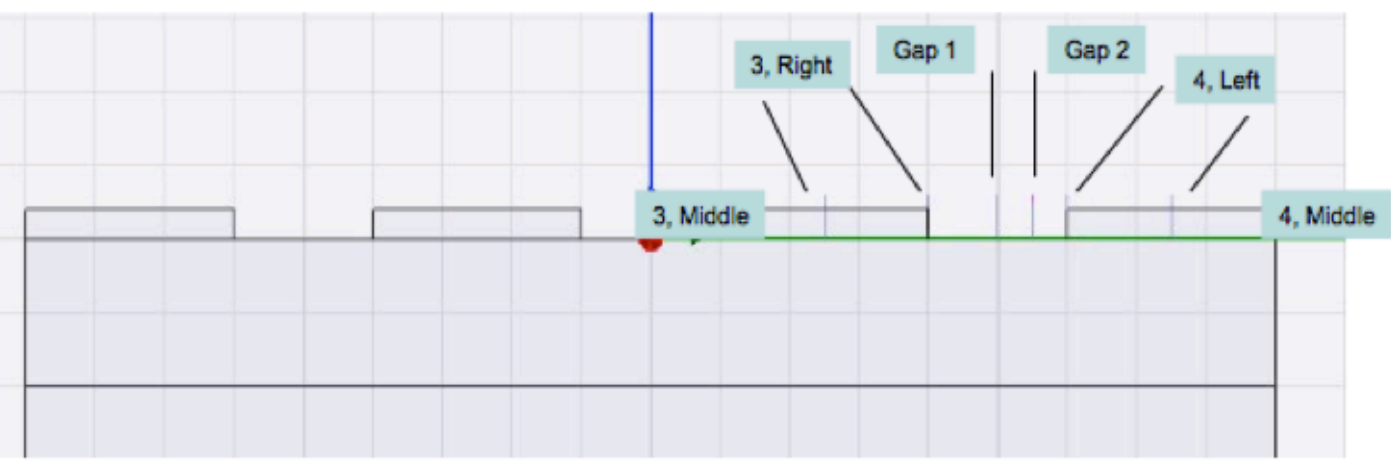

Fig 7. The four positions on the IDEA chip model that were evaluated for the magnitude of the electric field and the current density. All points were evaluated at 10 and $50 \mathrm{~nm}$ above the electrode surface.

The effect of the height of the electrodes on these parameters was also modeled using the same method as was the width of the electrode. Another geometric parameter examined was the pitch of the IDEA electrodes. A layer of a nonconducting polymer and layers of gold nanoparticles on top of the electrodes were also modeled to see if they could amplify the signals generated by the electrodes.

\subsection{Electrode spacing, electrode height, and electrode width models and} results. The spacing between our fabricated IDEs was $0.8 \mu \mathrm{m}$. Modeling was used to determine the optimal spacing between the electrodes that would result in a stronger electric field as evaluated at the points shown in Figure 7. In total, eight different gap widths were modeled. The widths chosen were $0.1,0.2,0.3$, $0.5,0.6,0.8,0.9$, and $1.0 \mu \mathrm{m}$. These values were chosen because they gave a 
sampling of the electric field and current density values for gap widths both larger and smaller than the gap currently used in our fabricated chips. Figure 8 is a comparison of the current density for four of the gap distances that were modeled. As the gap distance increased, the current density values decreased.
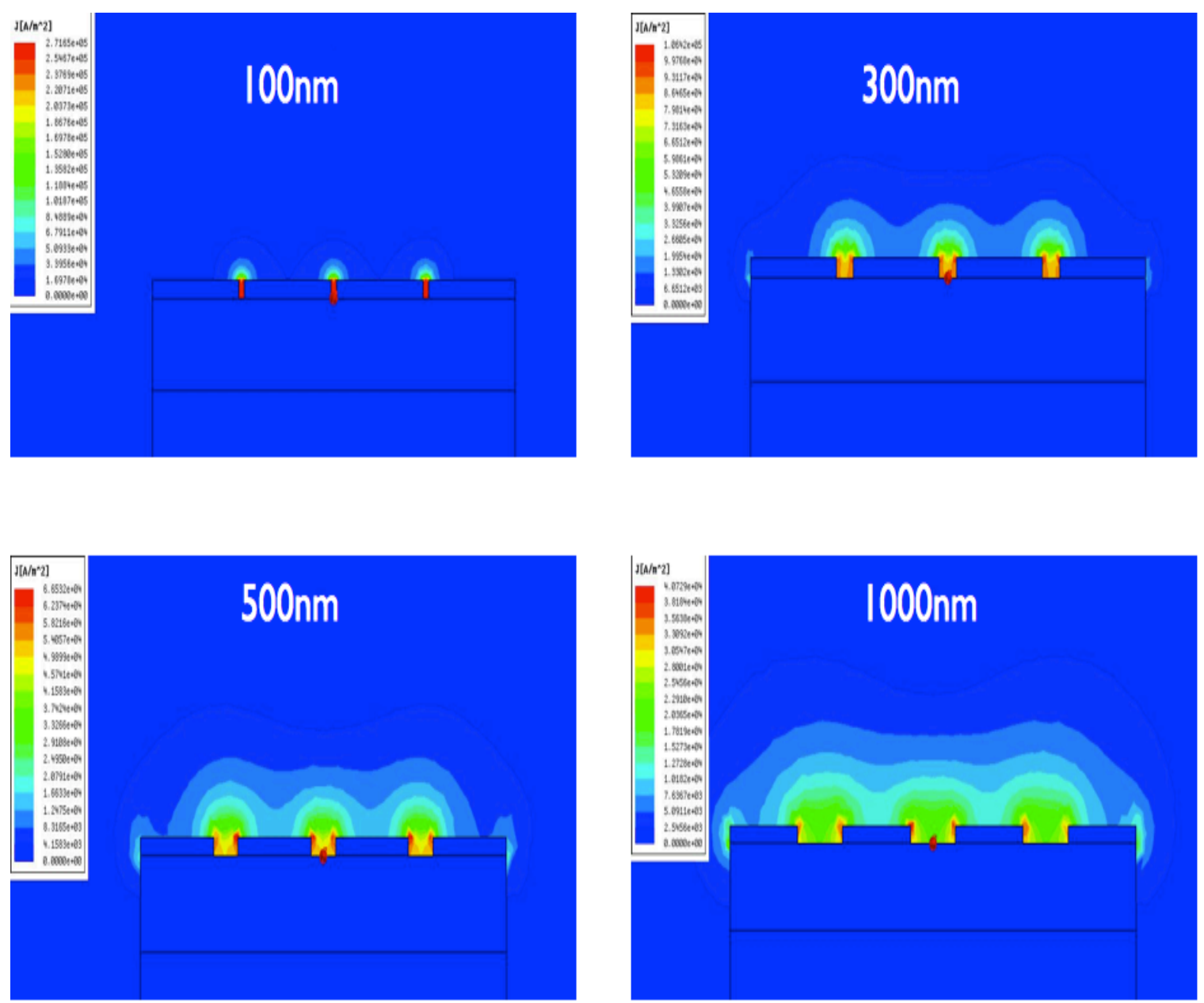

Fig 8. Comparison of current density distributions for four of the modeled gap distances. The four gap distances shown are 100,300,500, and 1000nm. The current density values decrease for increasing gap distances.

The results of the effect on the electric field of the various gap widths can be seen in Figure 9. The electric field and current density values exhibit an exponential decay for electrode spacings between $100 \mathrm{~nm}$ and $600 \mathrm{~nm}$ and 
flatline beyond that distance. As the spacing currently used in the chips is $800 \mathrm{~nm}$ and in that static region, it was evident that a decrease in gap distance had the potential to greatly enhance the electric field and current density values.

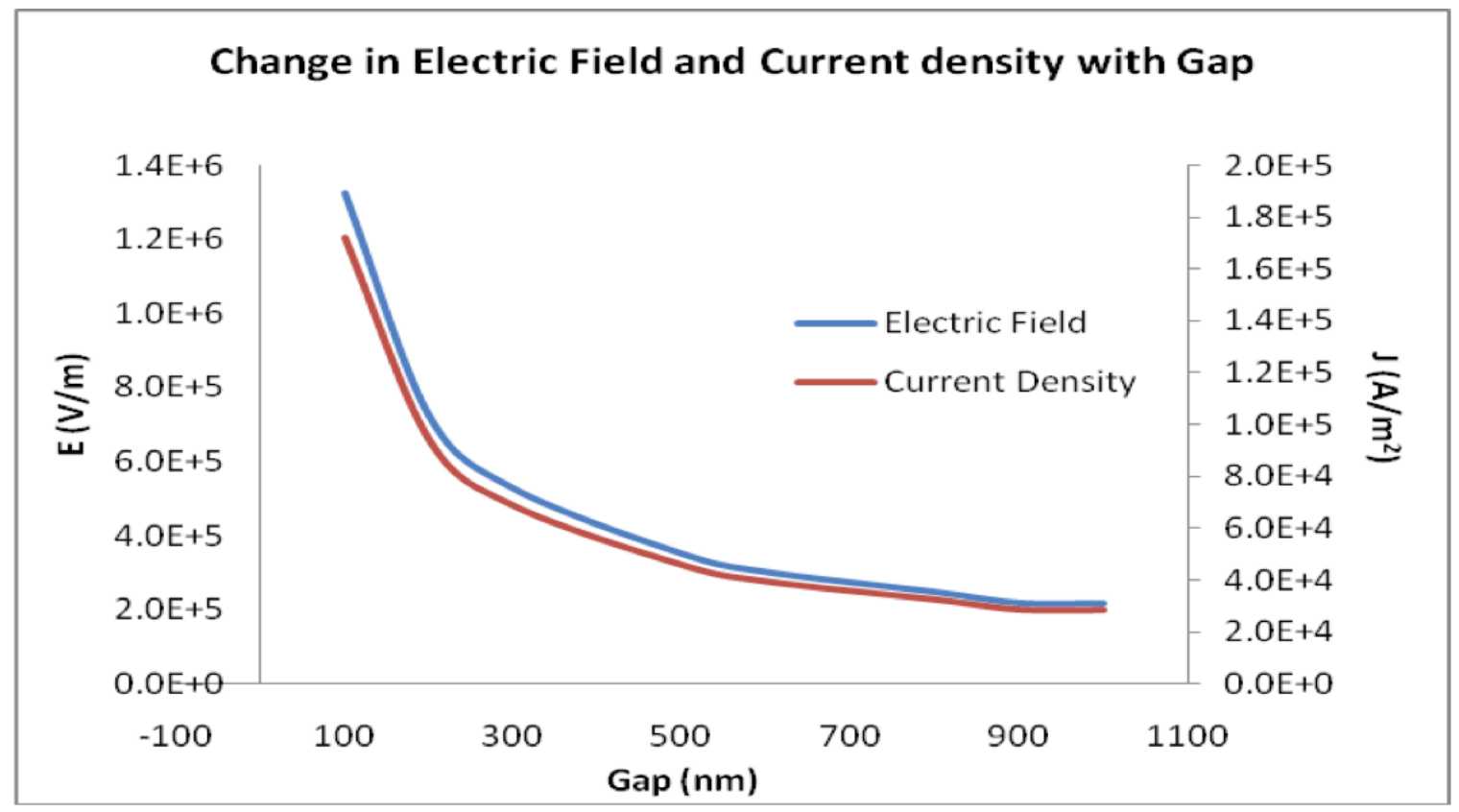

Fig 9. Graph of the decline in electric field and current density values with increasing gap distance. There is an exponential decay in values between 100 and $600 \mathrm{~nm}$ after which the values become static. All values were obtained at $10 \mathrm{~nm}$ from the electrode surface. This exponential decay was seen at every evaluated point on the electrodes.

The same effect can be seen in Figure 10. Keeping the width of the electrode constant at $1.5 \mu \mathrm{m}$ and the height of the electrode at $200 \mathrm{~nm}$, the electric field values at $10 \mathrm{~nm}$ from the electrode surface for gap distances of 100 , 300 , and $500 \mathrm{~nm}$ increase with decreasing gap distance. Though not shown, the effect on the current density values is the same.

The effect of the electrode height was explored. Heights of 10, 50, 200, and $500 \mathrm{~nm}$ were modeled. In these model the gap distance was kept at $500 \mathrm{~nm}$ 
and the electrode width kept at $1.5 \mu \mathrm{m}$. A gap distance of $500 \mathrm{~nm}$ was chosen arbitrarily; the purpose of the model was to keep other parameters constant while the height of the electrode was altered. The resulting effects on the electric field values at a distance of $10 \mathrm{~nm}$ from the electrode surface are in Figure 10. The electrode height has no effect on the electric field values. Though not shown, the effect of the change in electrode height on the current density values is the same; there is no change in the values.

Finally, the effect on the electric field at 10nm from the electrode surface for different electrode widths were modeled. Widths of $500,750,1000$, and 1500 $\mathrm{nm}$ were used while the electrode height was kept constant at 200nm. These widths were used with gap distances of 100,300 , and $500 \mathrm{~nm}$. The results can be seen in Figure 10. The electric field values are static for every width for every gap distance. The electrode width has no effect on the electric field values. 


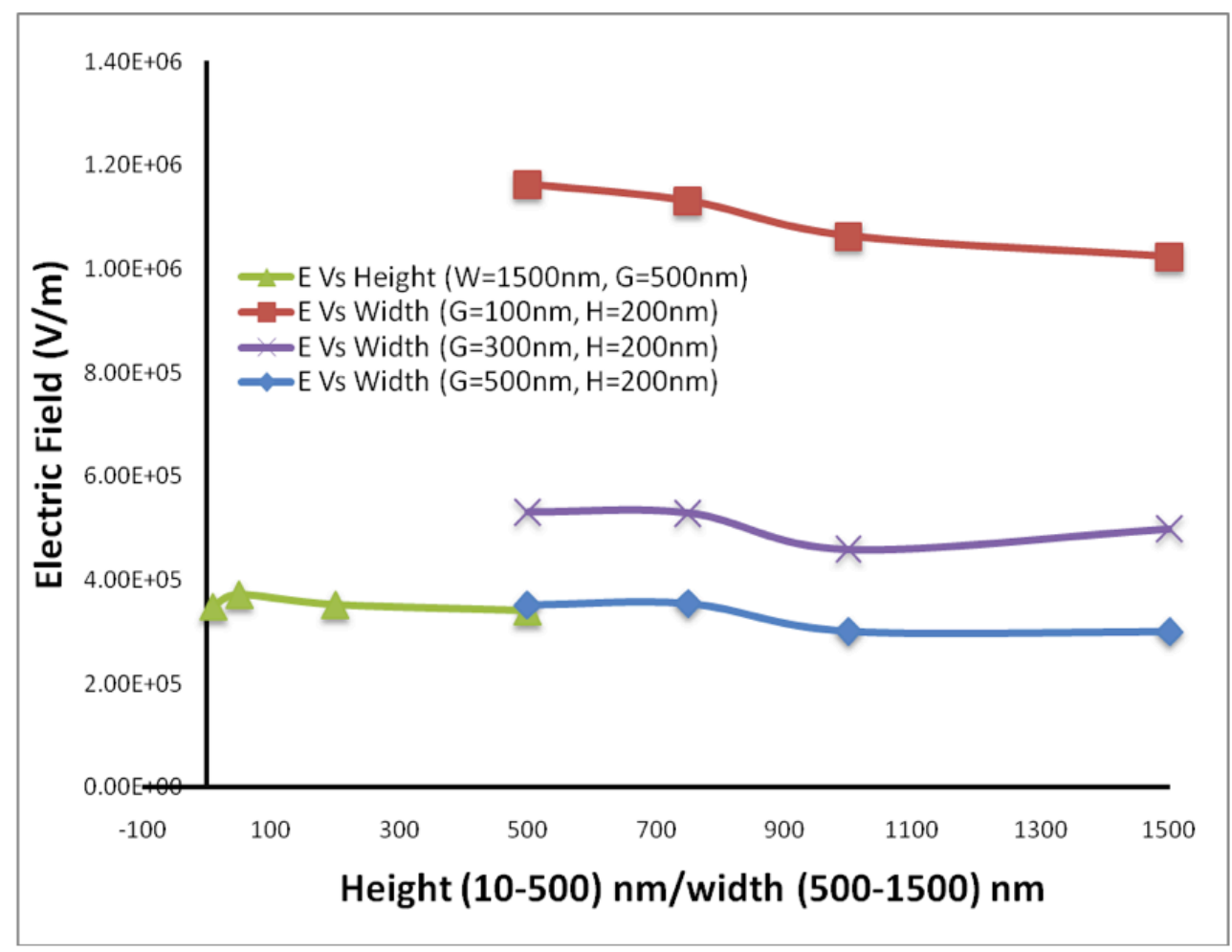

Fig 10. Graph of change in electric field values with changes in electrode height, electrode width, and gap distance. There is no change in the electric field with a change in electrode height or width, but there is an increase in the electric field with decreasing gap distance.

2.3 Pitched electrodes. Two different geometries using pitched electrodes were created in Maxwell and the electric field and current density values were obtained. The first pitched electrodes had 90 degree edges up to a height of 100 $\mathrm{nm}$ and 45 degree pitch for the remaining $100 \mathrm{~nm}$. Figure 11 contains an image of the electrodes as well as the points evaluated for comparison and the resulting changes in the electric field and current density at $10 \mathrm{~nm}$ from the electrode surface. Though not shown, the data from $50 \mathrm{~nm}$ from the electrode surface exhibits the same trend. 
a)
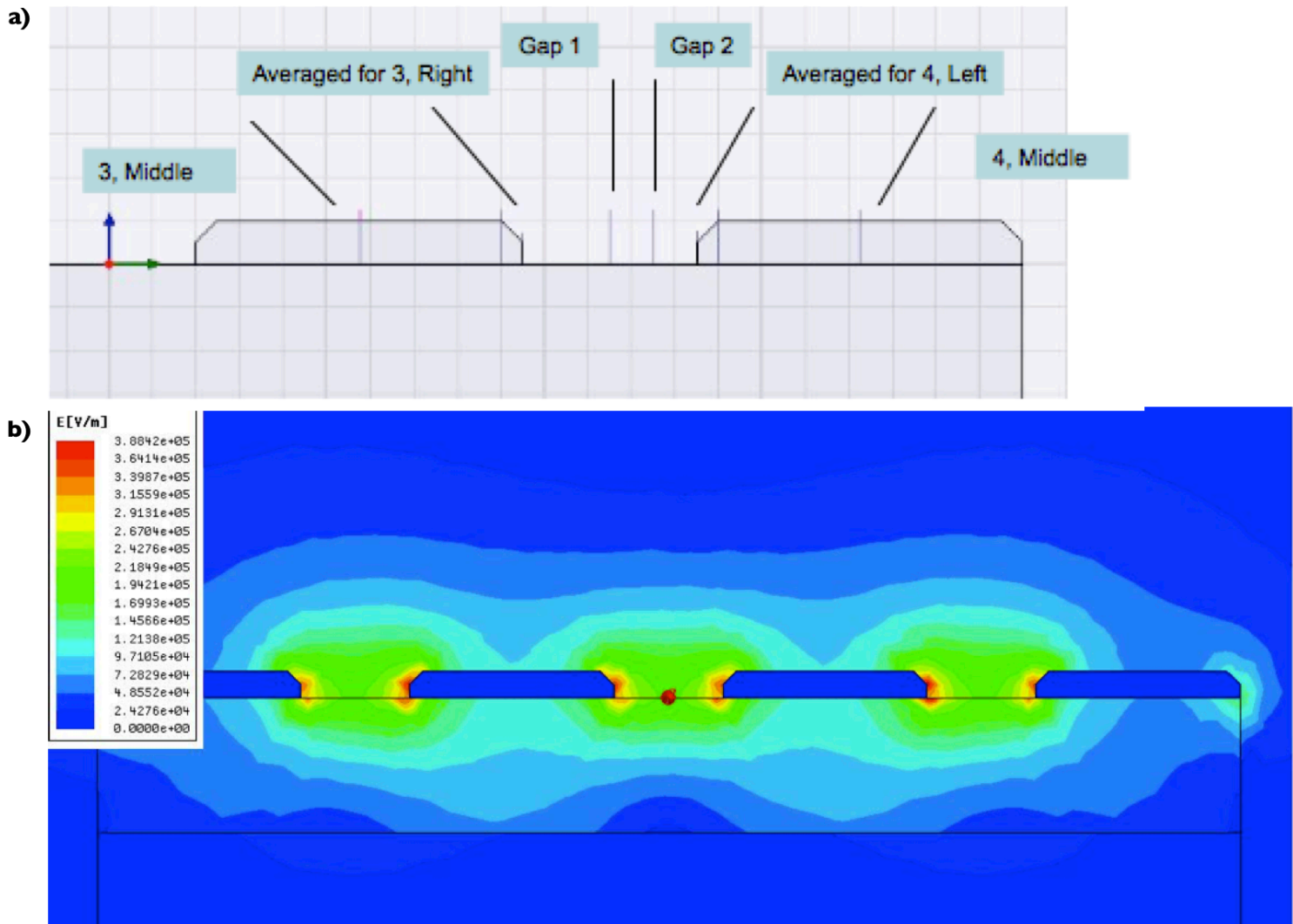

C) \begin{tabular}{r|}
$J\left[A / m^{\wedge} 2\right]$ \\
$5.0170 e+24$ \\
$4.7034 e+04$ \\
$4.3899+04$
\end{tabular}

$4.3899 e+04$
$4.0763 e+04$

3. $7628 \mathrm{e}+64$

3. $4492 e+84$

3. $1356 e+84$

2. $6221 \mathrm{e}+04$

2. $5085 e+94$

$2.1949 e+04$

1. $8814 e+84$

$1.5678 \mathrm{e}+84$

1. $2543 e+04$

9. $4069 \mathrm{e}+03$

6. $2713 e+03$

$3.1356 \mathrm{e}+03$

$0.6000 \mathrm{e}+00$

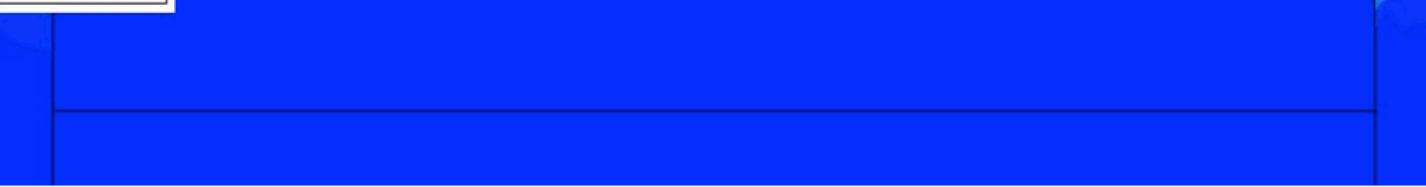


d)

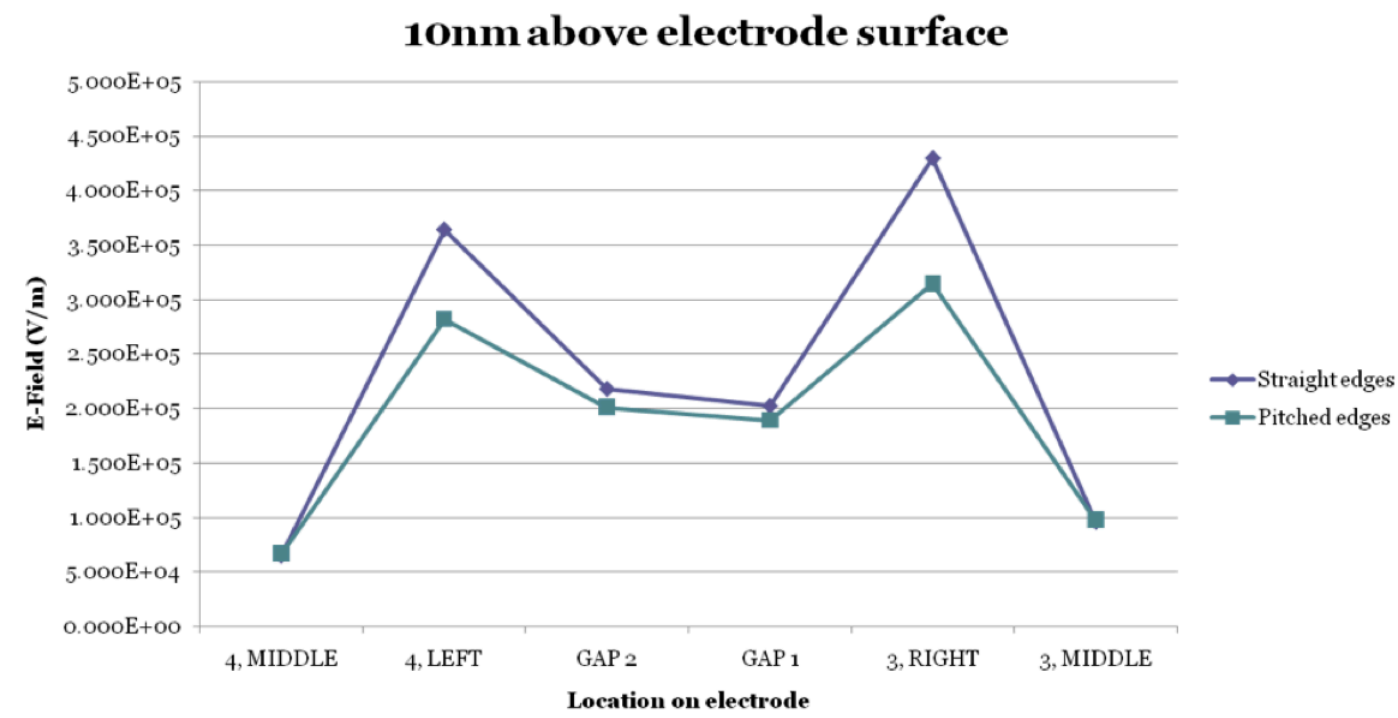

e)

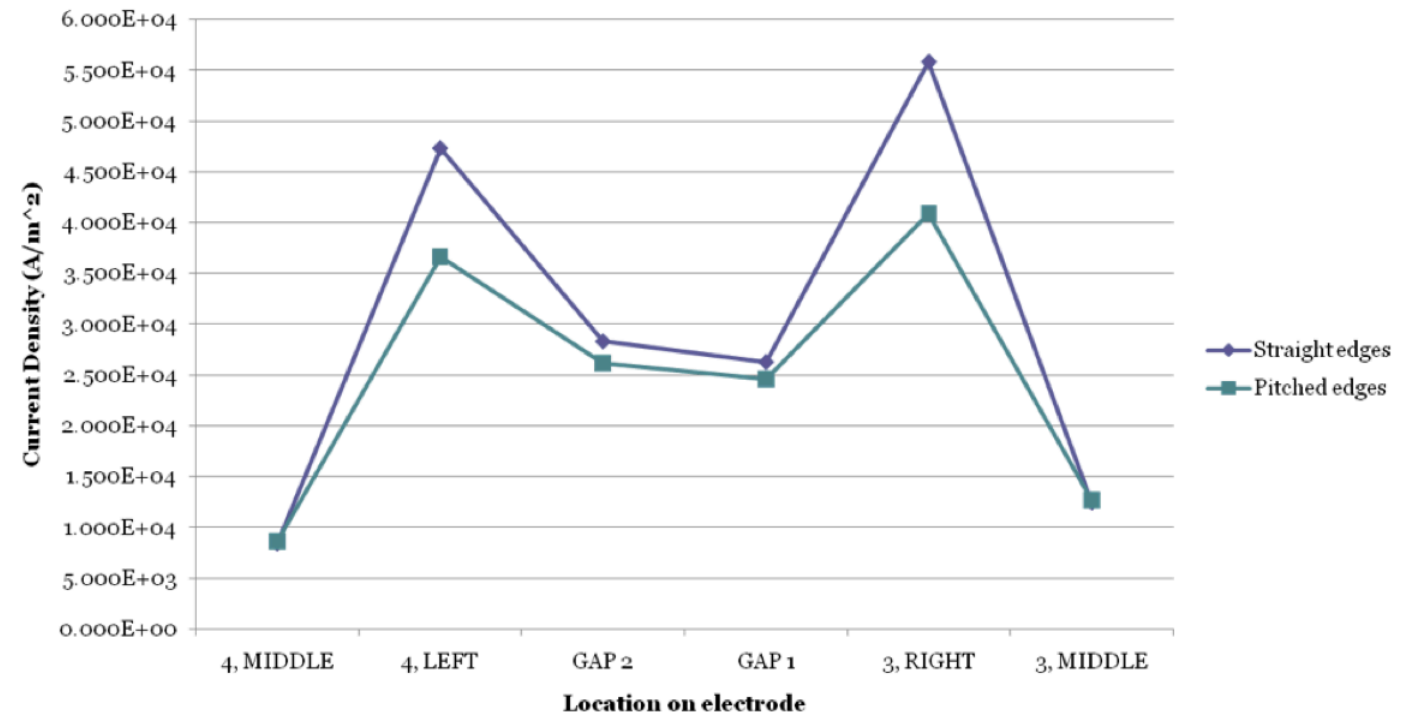

Fig 11. Results of first pitched geometry. a) The positions that were evaluated for this geometry. b) The electric field and c) current density distributions. d) The electric field and e) current density values as evaluated for every point in a) at a distance of $10 \mathrm{~nm}$ from the electrode surface. The electric field and current density values are stronger for the unmodified electrode geometry.

The second pitched geometry contained electrodes that were pitched at 45 degree over a height of $200 \mathrm{~nm}$. Figure 12 contains images of the positions used to evaluate the impact of such a geometry on the electric field and current density values. The data values represented in the graphs were obtained at 10 
$\mathrm{nm}$ from the electrode surface and the values obtained from $50 \mathrm{~nm}$ from the electrode surface exhibited the same trend.

a)

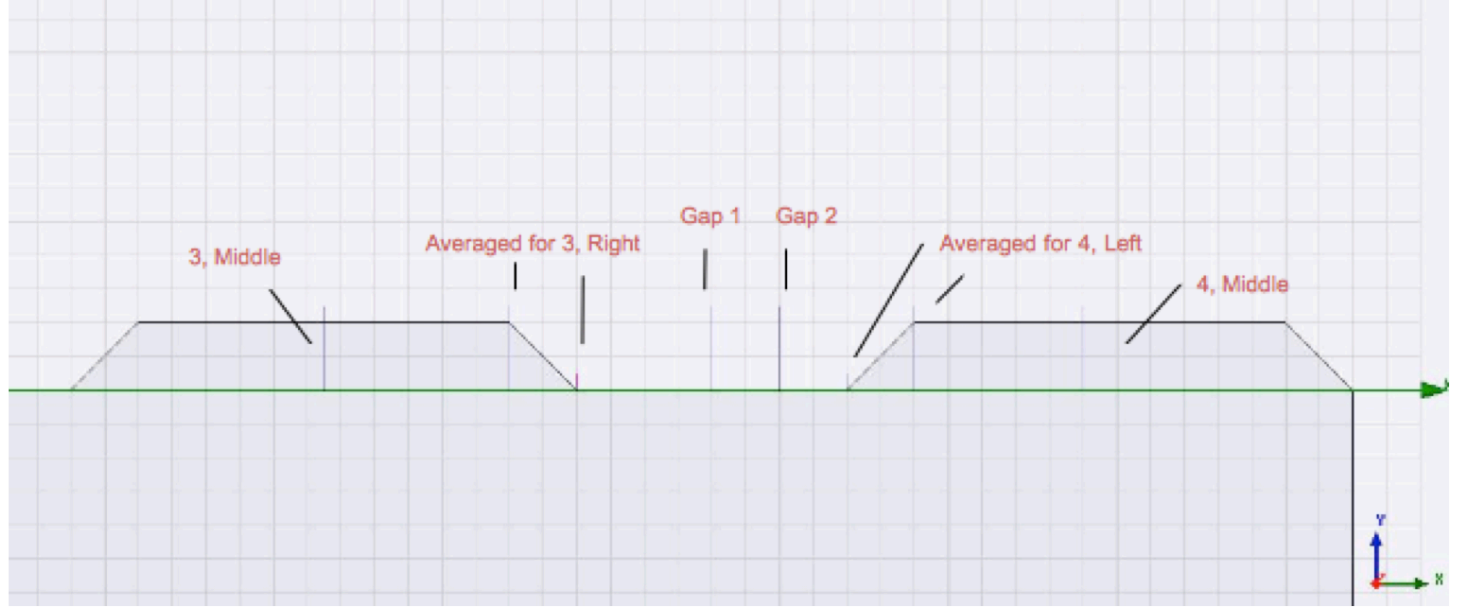

b)

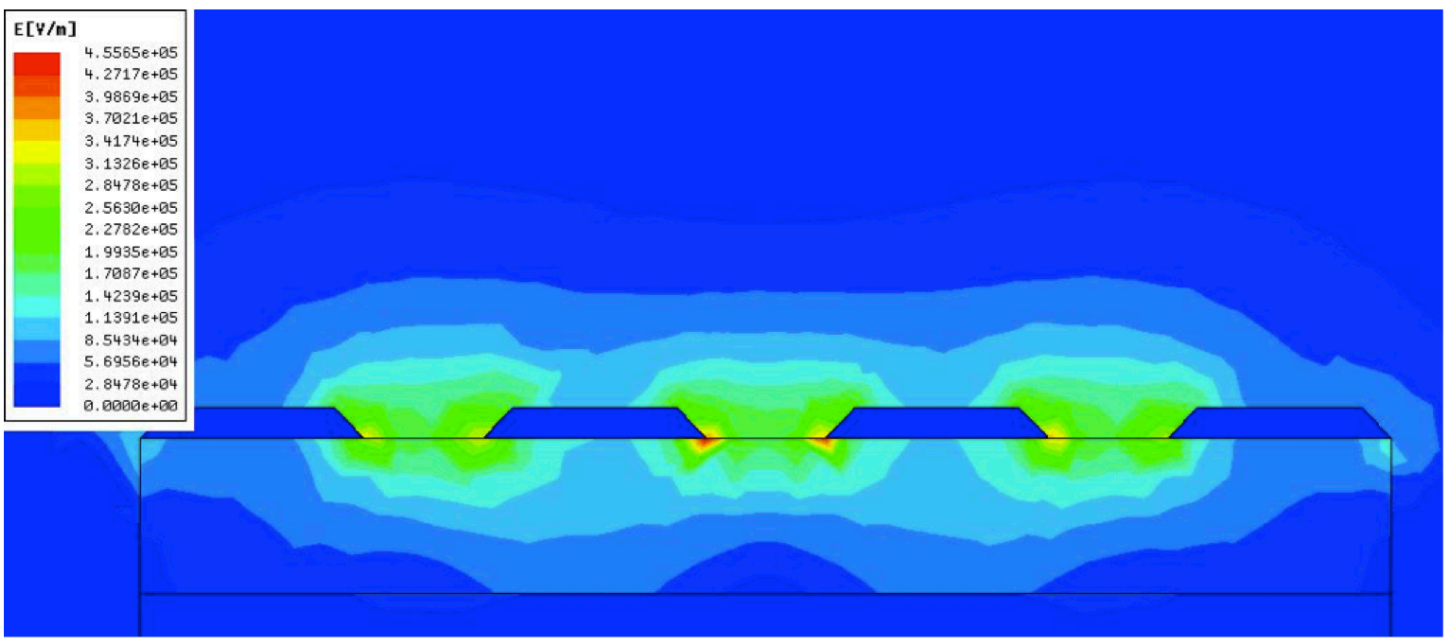

C) $J\left[A / m^{\wedge} 2\right]$

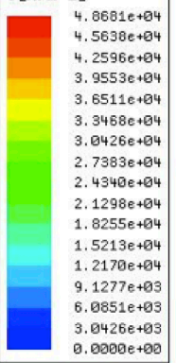

(1)

.

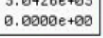
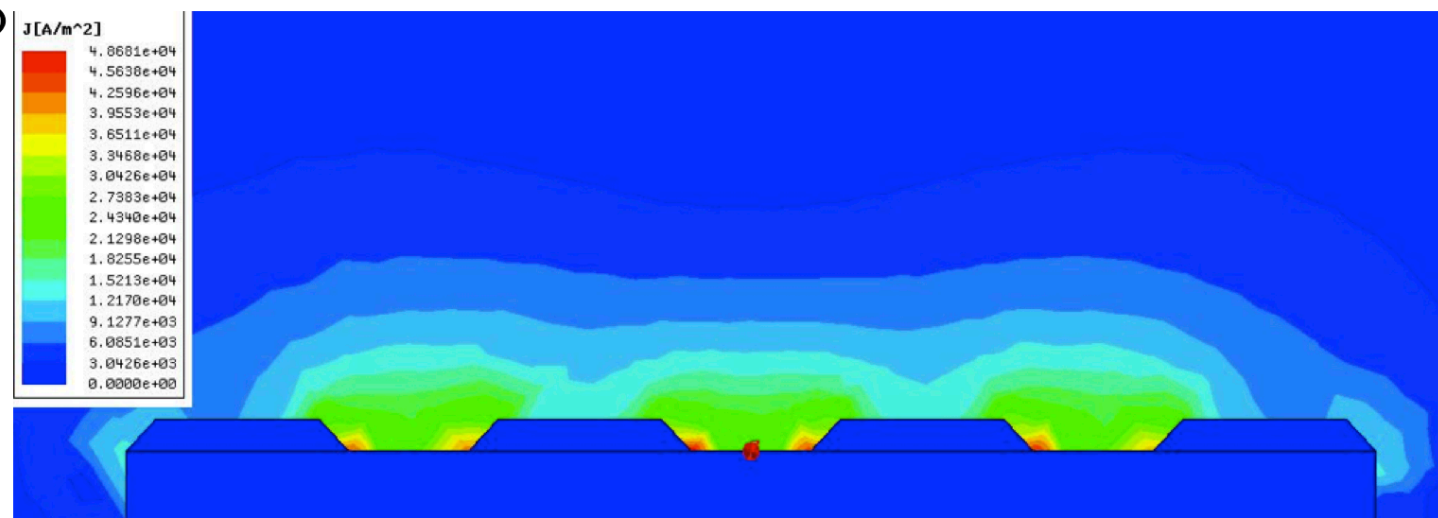
d)

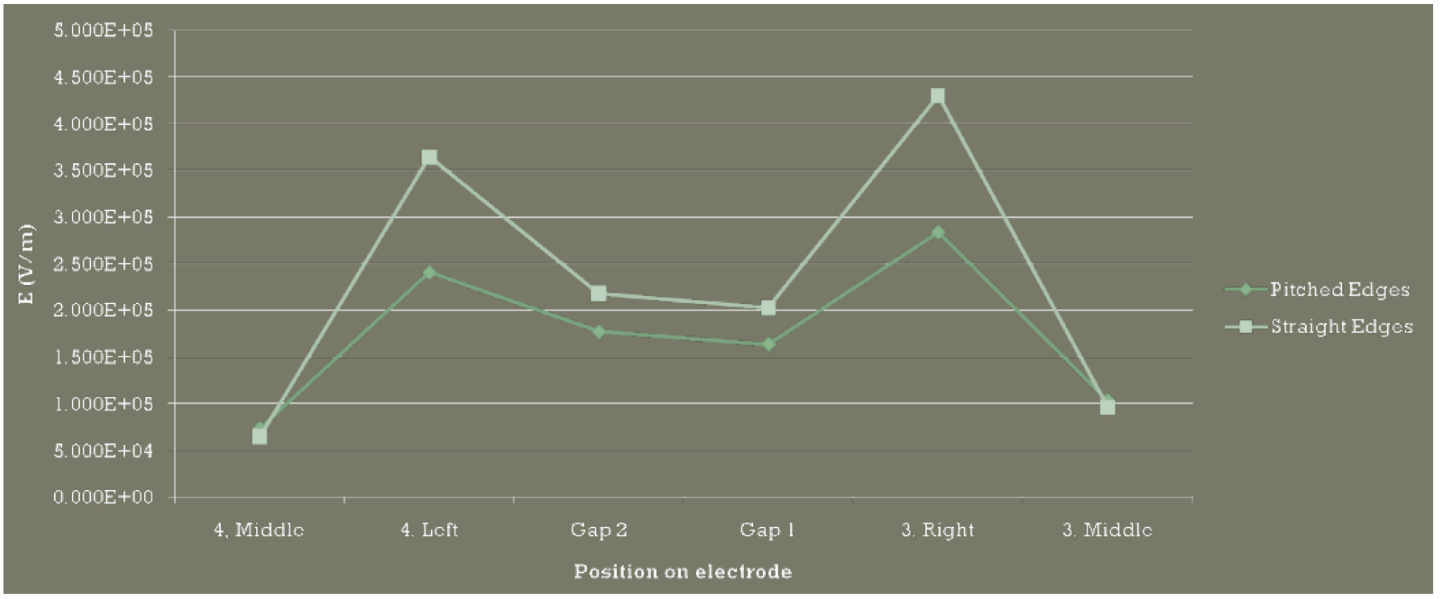

e)

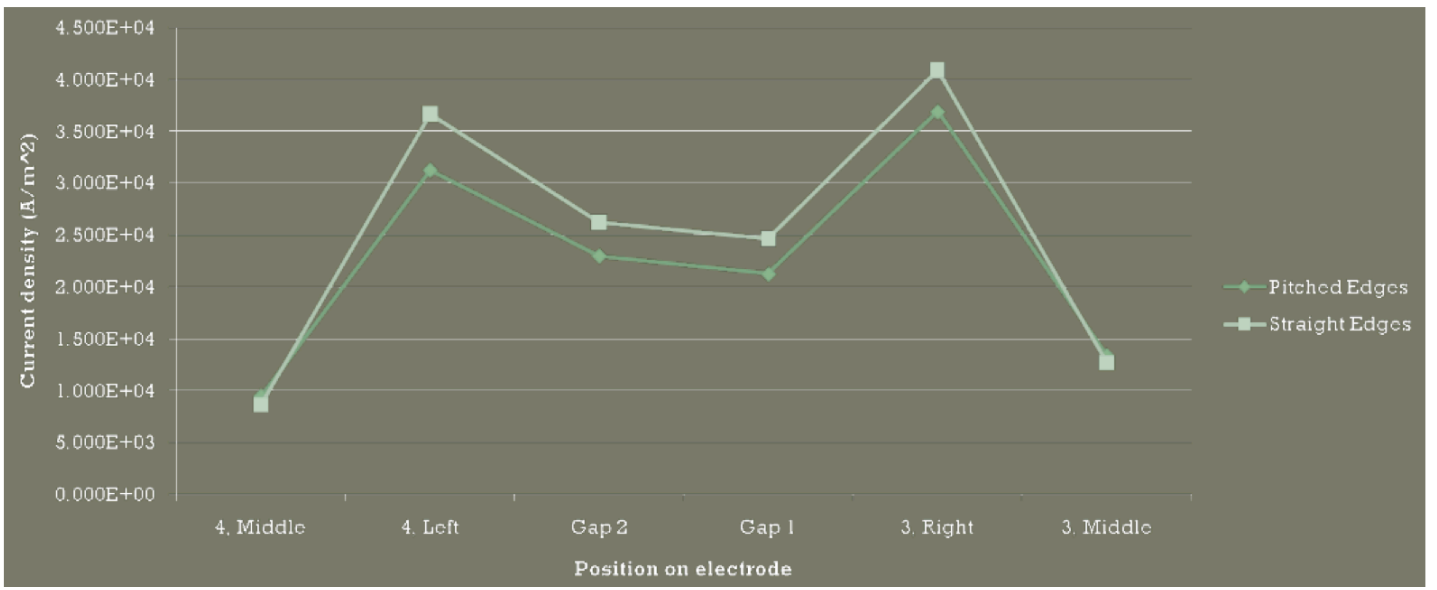

Fig 12. Results of the second pitched geometry. a) The positions that were evaluated for this geometry. b) The electric field and c) current density distributions. d) The electric field and e) current density values as evaluated for every point in a) at a distance of $10 \mathrm{~nm}$ from the electrode surface. The electric field and current density values are stronger for the unmodified electrode geometry.

Neither of the pitched geometries increased the electric field or current density values. They did not largely affect either set of values. It was determined that currently used electrode geometry with 90 degree edges was the optimal geometry.

2.4 Nonconducting polymer over the electrodes. A model was created in which a $100 \mathrm{~nm}$ thick layer of polyamide, a nonconducting polymer, was coated 
on the electrodes and the gap between the electrodes. Polyamide is a polymer often used in electrochemical biosensors to bind analytes at the electrodes. It is usually developed on the electrodes over a thickness range between 10 and 100 $\mathrm{nm}$. It has a relative permittivity of 4.3 and a conductivity of 0 . The results of the effects of the polyamide on the electric field and current density can be seen in Figure 13. 
a)

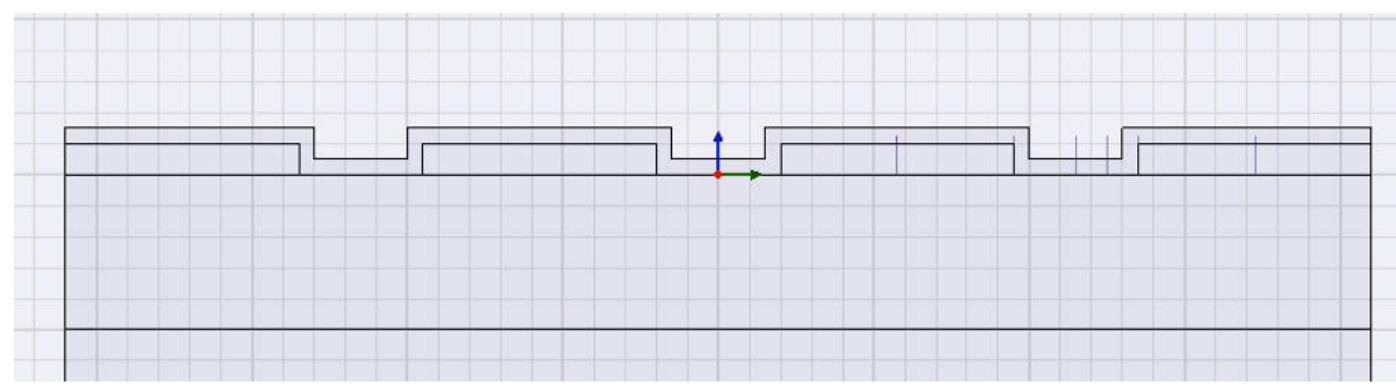

b)

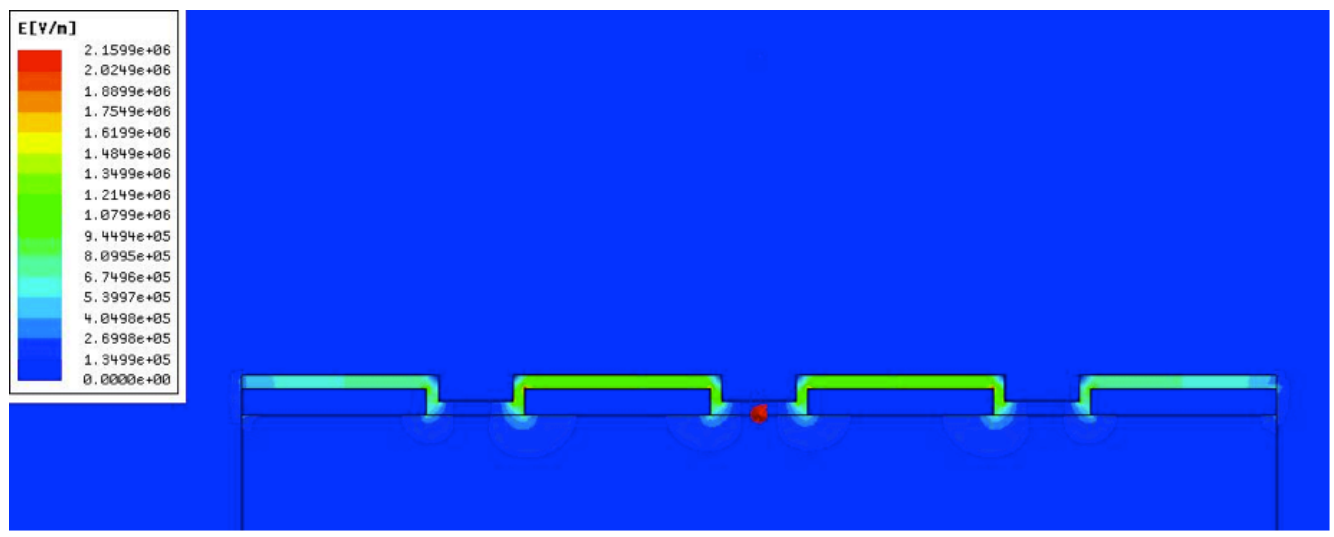

c)

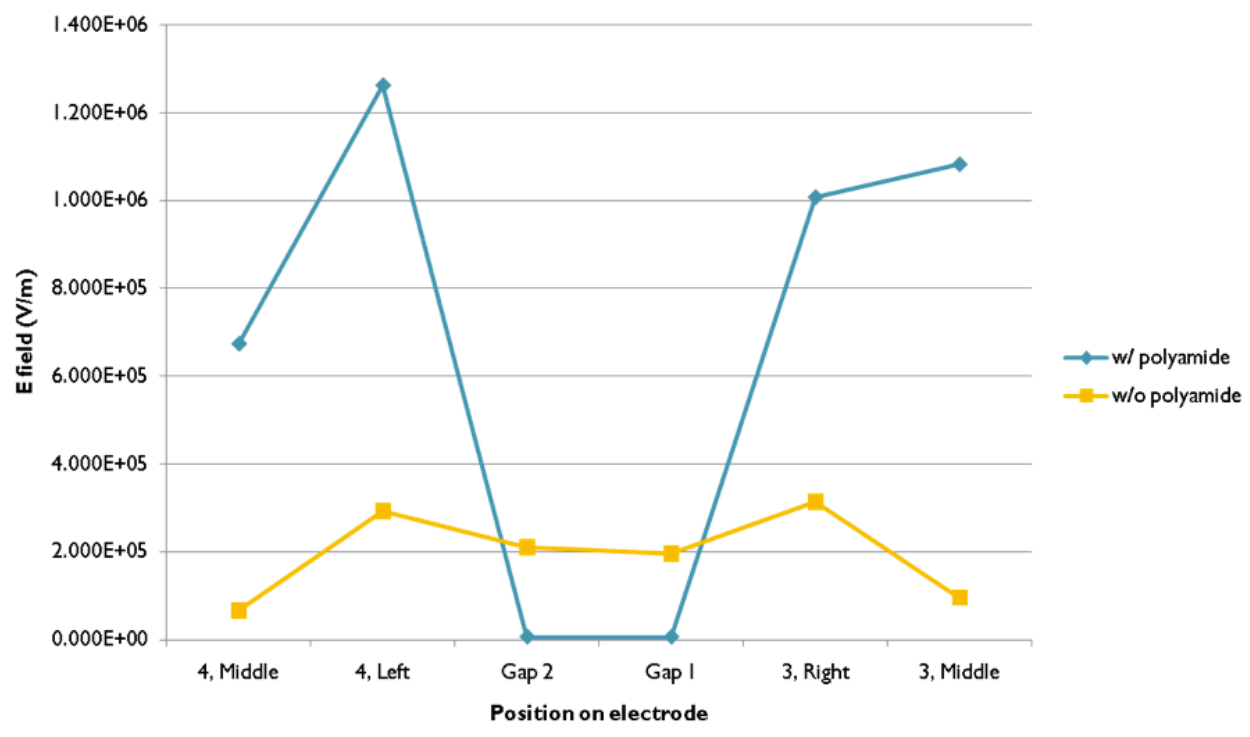

Fig 13. The results of the nonconducting polyamide layer. a) The positions that were evaluated for this model. b) The electric field distribution for the layer. c) The values of the electric field within the polyamide layer are enhanced by an order of magnitude. The electric field values in the gap, measured beyond the layer, are zero. The polyamide layer has the potential to greatly enhance the signal generated by an electrical biosensor. 
Within the polyamide layer the electric field is greatly enhanced. The strength of the field increase by an order of magnitude. This is seen in the evaluation of the points outside of the gaps. The points evaluated in the gaps show an electric field reduced to zero as they are evaluated in a space not contained with the layer of polyamide. This model suggests that a thin layer of polyamide would be an effective way to amplify the signal generated by an electrical biosensor. Biomolecular interactions take place within 10 to $100 \mathrm{~nm}$ of the electrode surface. A $100 \mathrm{~nm}$ thick layer of polyamide would enhance all interactions taking place within this range.

2.5 Nanoparticle enhancement of electrodes. Nanoparticles, like polyamide layers, are a commonly used material to enhance the signal generated by electrical biosensors. Several models were created to evaluate the effect on these materials on the electric field and current density. The first model was based on a single layer of polyamide nanoparticles $20 \mathrm{~nm}$ in diameter. The nanoparticles were placed all over the electrodes and in the gap at a low density. Figure 14 contains the results of the model.

The nanoparticles enhance the electric field and current density on the edge of the exterior electrode, labeled as 4 , left in the figure, at a distance of 10 $\mathrm{nm}$ from the electrode surface but the same values for the interior electrode edges are reduced. This reduction in values could have a large effect. There are 37 interior IDEs and only two exterior electrodes. At $50 \mathrm{~nm}$ from the electrode 
surface the electric field and current density values have reached roughly the same values and there is no enhancement of either signal observed at the edge of the interior electrode. The nanoparticles do not seem to have an effect in the gap area. Those positions are evaluated well above the nanoparticle layer and are the same for either geometry.

a) $E[Y / m]$
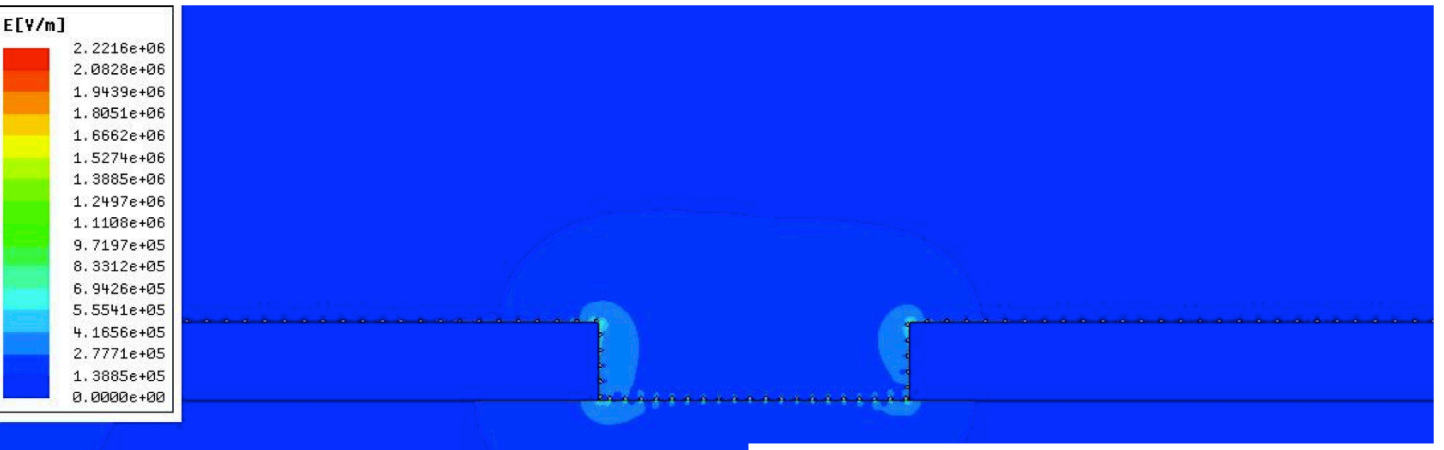

b)
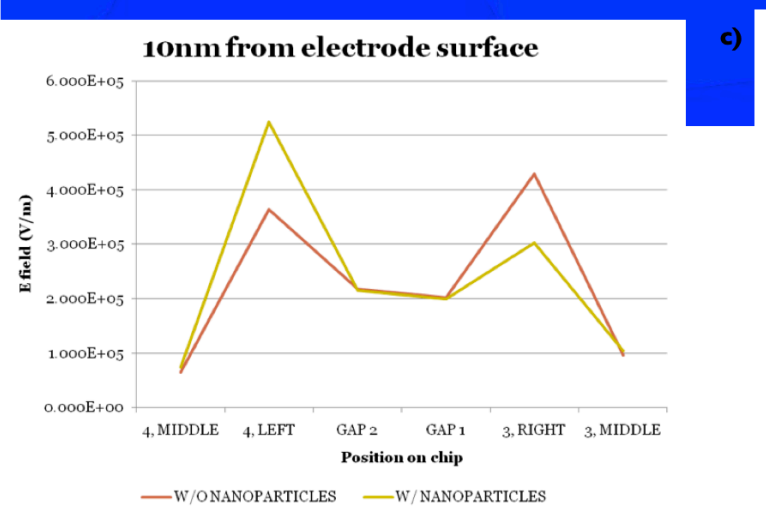

d)

10nm from electrode surface

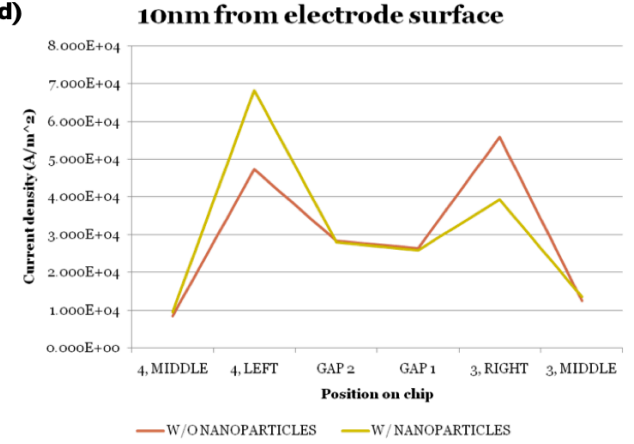

e)

$50 \mathrm{~m}$ from electrode surface
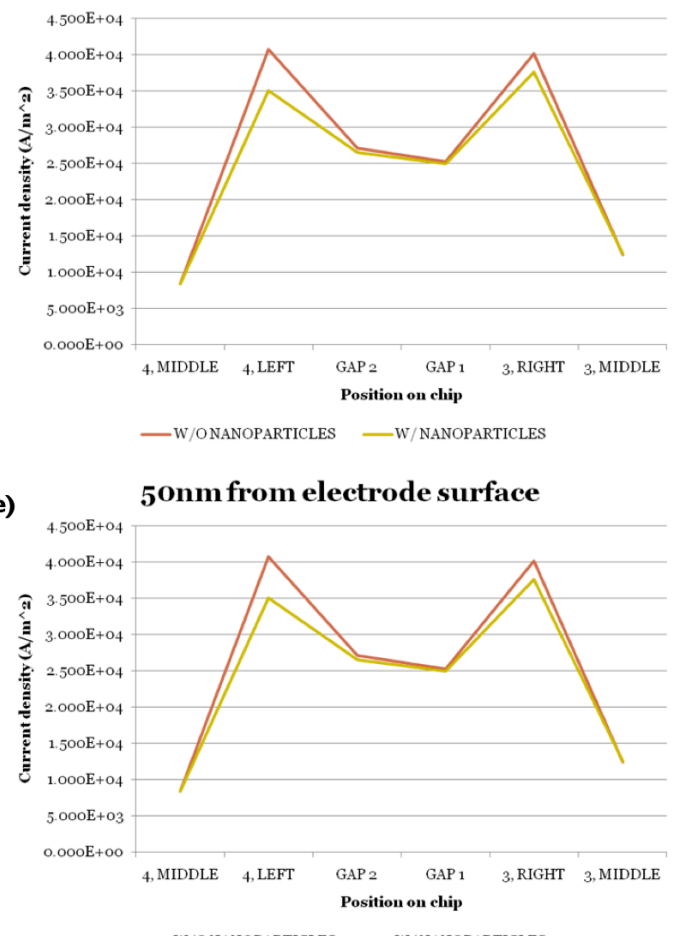

-W/ONANOPARTICLES —W/NANOPARTICLES 
Fig 14. The results of a single layer of polyamide nanoparticles at a low density. a) The electric field distribution of the nanoparticles. b,d) The electric field and current density values are increased at one position at $10 \mathrm{~nm}$, but $\mathrm{c}, \mathrm{e})$ they are no longer enhanced at a distance of $50 \mathrm{~nm}$.

Next, a higher density of polyamide nanoparticles were placed on the electrode surface and in the electrode gaps and the electric field and current density values were compared. Figure 15 contains the results at a distance of 50 $\mathrm{nm}$ from the electrode surface. Though the values obtained at $10 \mathrm{~nm}$ from the electrode surface are not represented in the figure, they follow the same pattern as the values obtained at a distance of $50 \mathrm{~nm}$. The addition of the nanoparticles greatly reduces the current density values, but enhances the electric field values. Polyamide is an insulating polymer so the effect on the current density is to be expected. 
a)

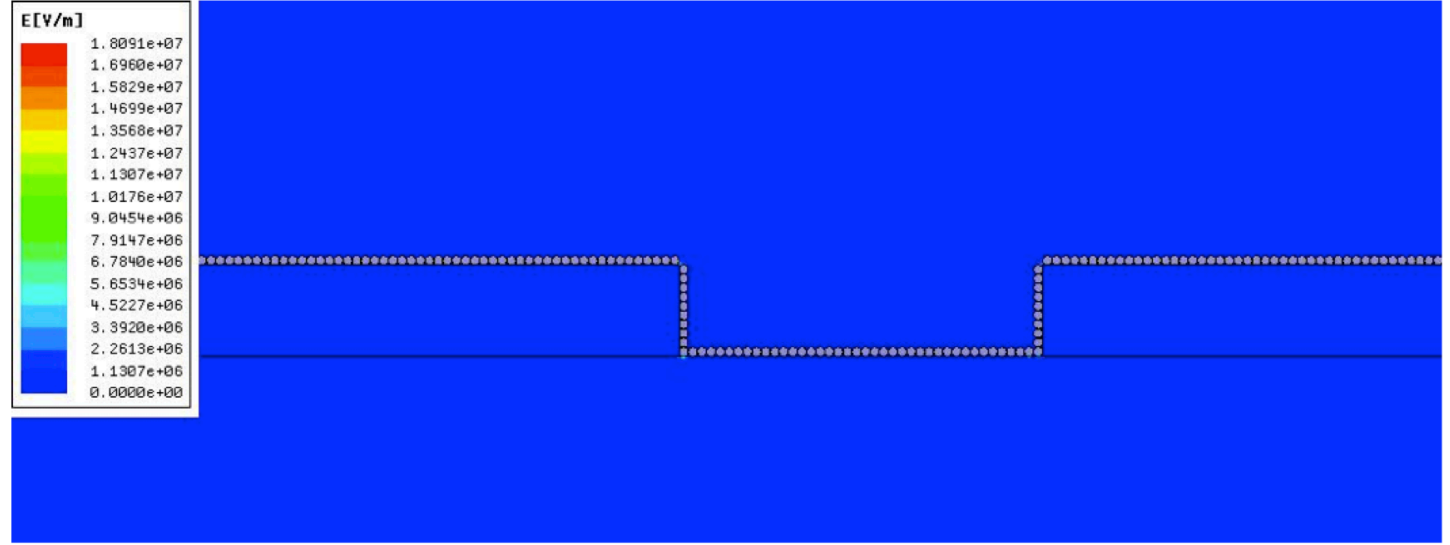

b)

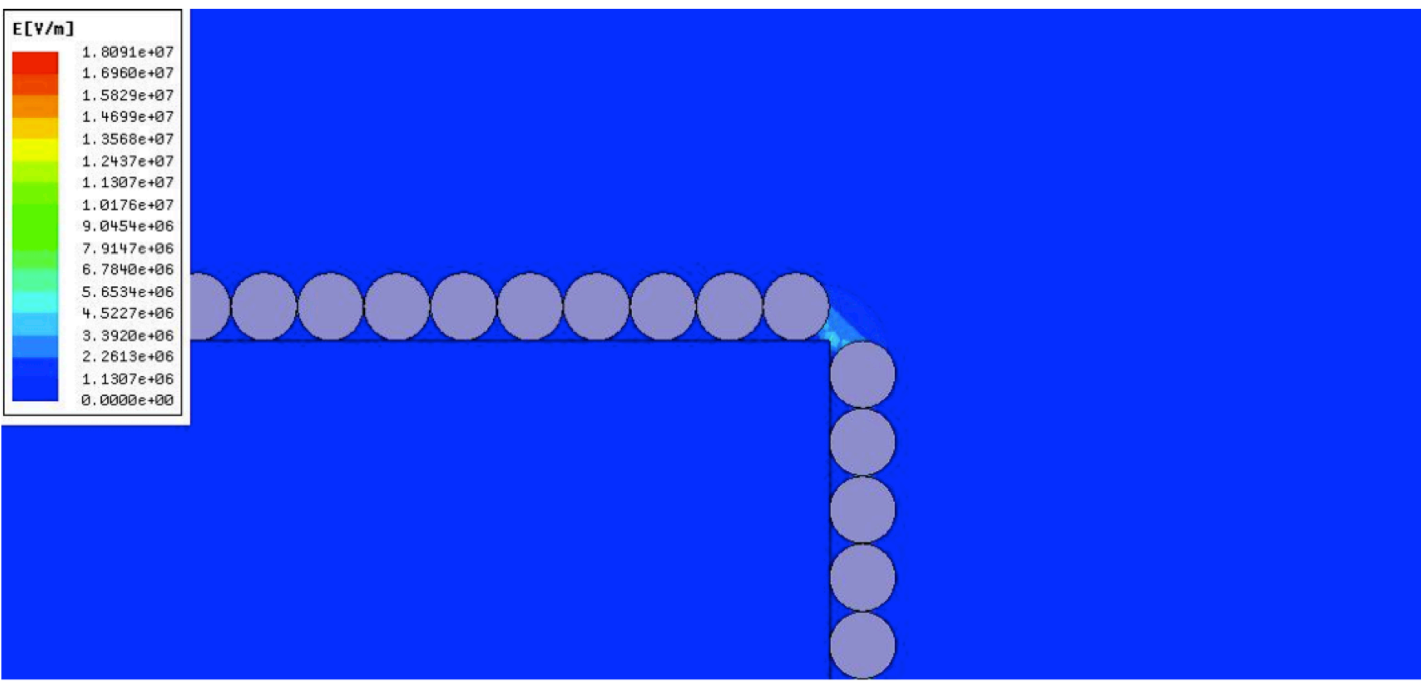


c)

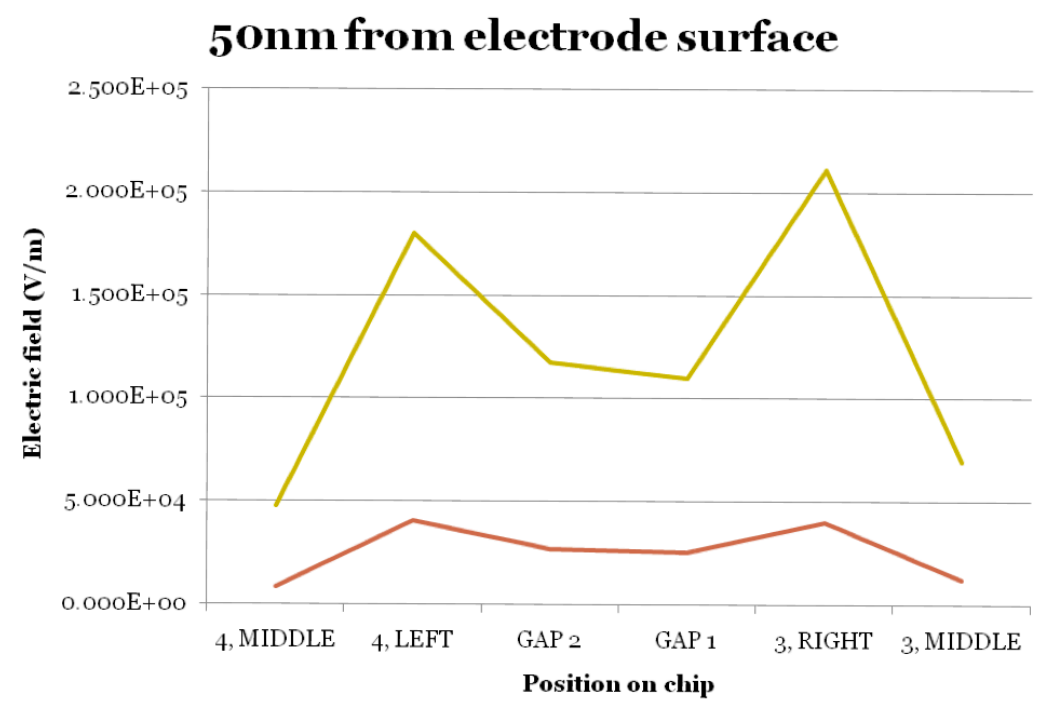

-W/ONANOPARTICLES —W/NANOPARTICLES

d)

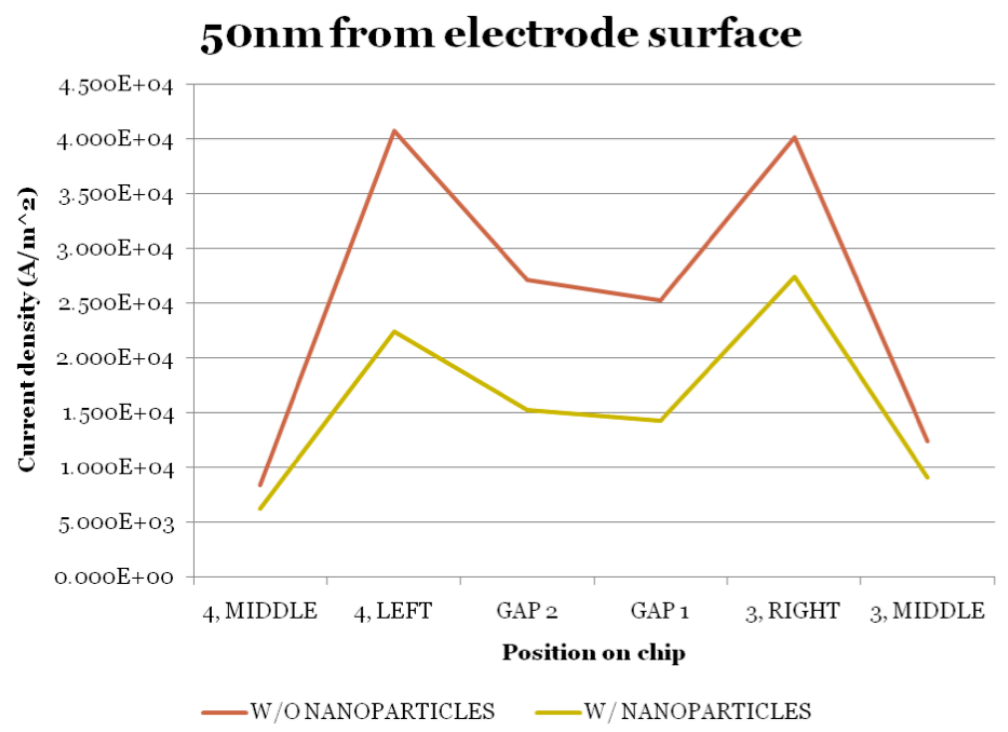

Fig 15. Results of a high density single layer of polyamide nanoparticles. a) the electric field distribution with b) a closeup. c) The electric field and d) current density values for the evaluated points at $50 \mathrm{~nm}$. The electric field values are increased while the current density is decreased.

A double layer of polyamide nanoparticles on the electrodes and gaps at

high and low densities were also modeled. The low density results are in Figure 16 and the high density results are in Figure 17. The current density values are lowered by the addition of the low density double layer of nanoparticles. The 
electric field values for the edge of the exterior electrode are enhanced at a distance of $10 \mathrm{~nm}$ from the electrode surface, just as they were for the low density single layer of polyamide electrodes. However, unlike the low density single layer of nanoparticles, the electric field values for the edge of the interior electrodes are not reduced. At a distance of $50 \mathrm{~nm}$ from the electrode surface the electric field values for this low density double layer of nanoparticles reached the same magnitude as the electric field values for the unmodified electrodes. The current density values are lowered by the addition of the low density double layer of nanoparticles. They remain reduced at every evaluated position and at distances of $10 \mathrm{~nm}$ and $50 \mathrm{~nm}$ from the electrode surface.

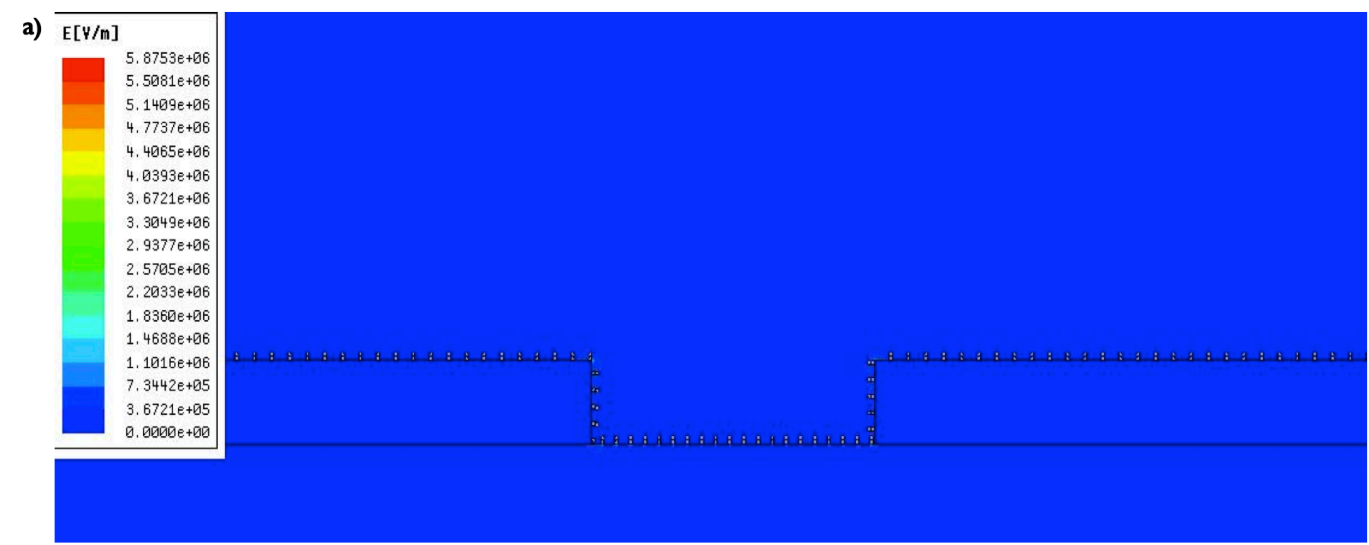

b)

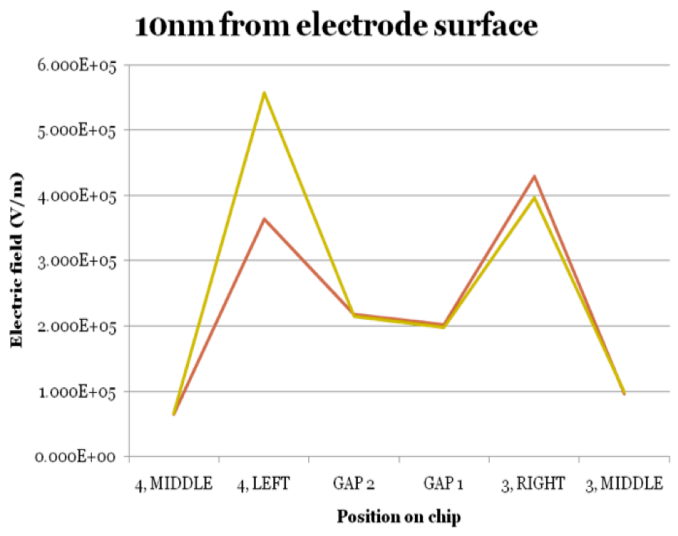

—W/ONANOPARTICLES —W/NANOPARTICLES c)

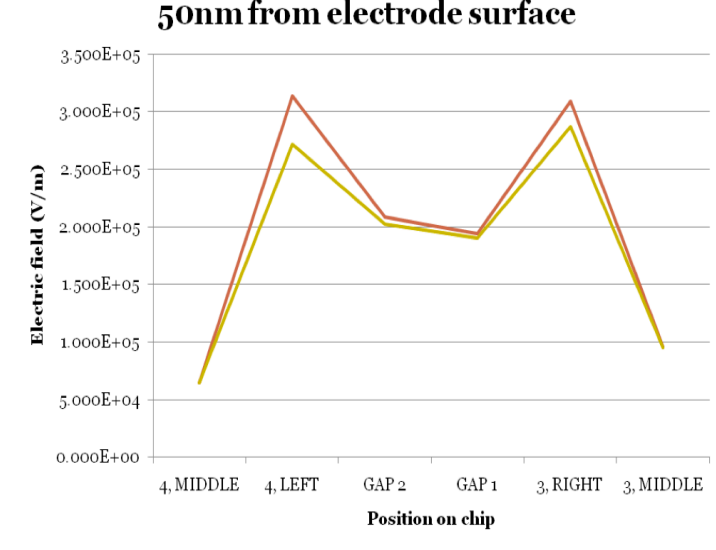

-W/ONANOPARTICLES —W/NANOPARTICLES 

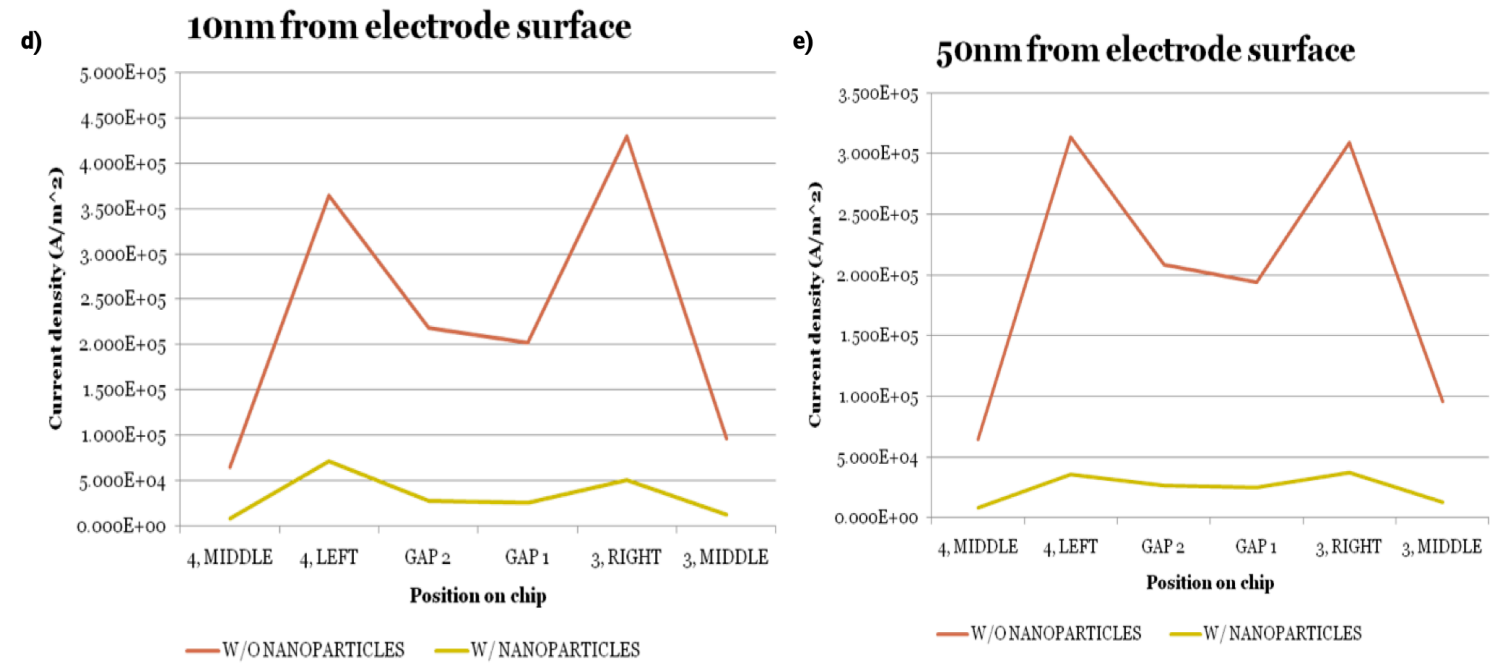

Fig 16. Results of double layer of polyamide nanoparticles at low density. a) The electric field distribution. b,c) The electric field is enhanced at one position at a distance of $10 \mathrm{~nm}$ from the electrode surface but the values are no longer increased at a distance of $50 \mathrm{~nm}$. d,e) The current density values are larger without the presence of nanoparticles.

The electric field values were obtained and compared for a high density double layer of nanoparticles at a distance of $10 \mathrm{~nm}$ from the electrode surface. The values are reduced at every evaluated position. Though not shown, the electric field values at a distance of $50 \mathrm{~nm}$ exhibited the same trend as did the current density values at the same distances. 
a)

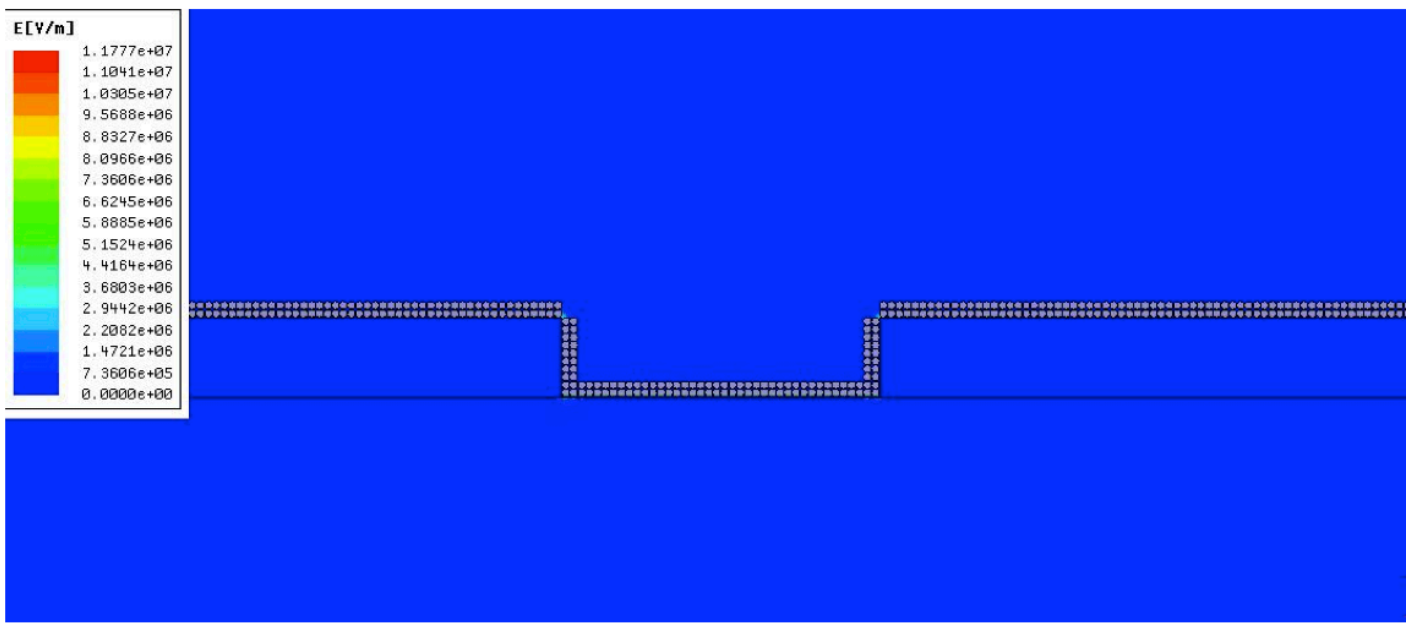

b)

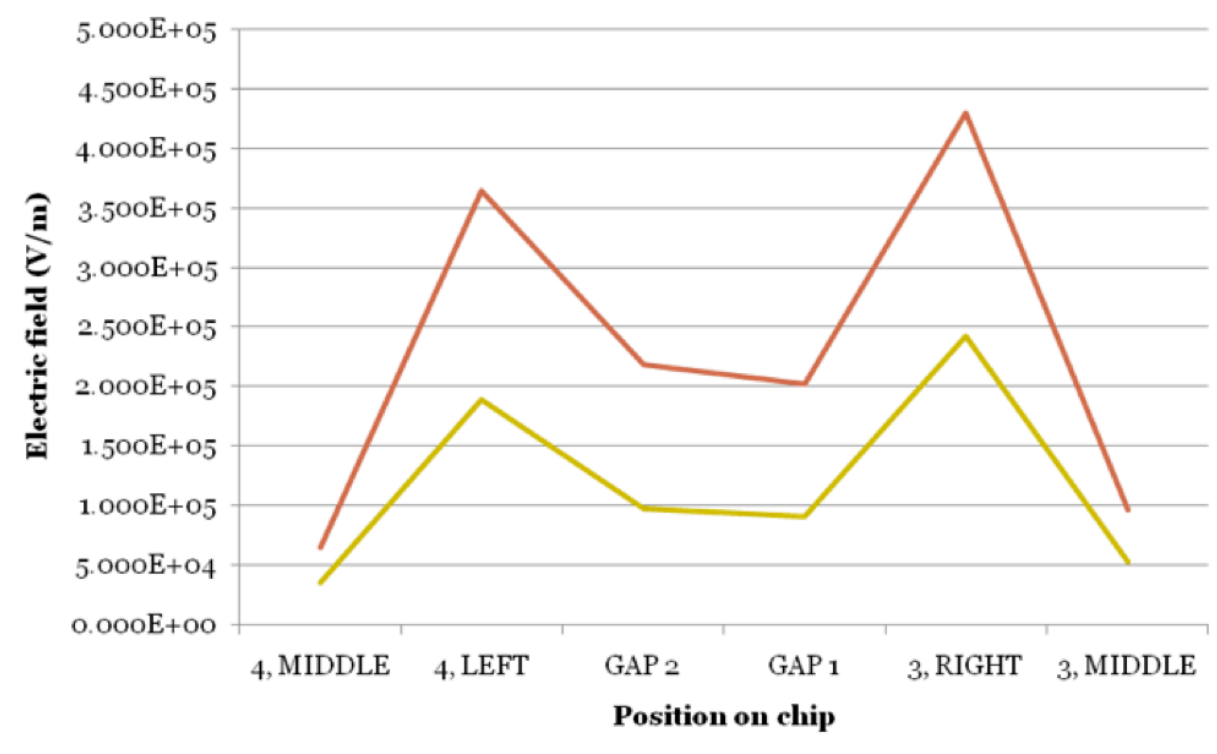

-W/ONANOPARTICLES $-\mathrm{W} /$ NANOPARTICLES

Fig 17. Results of a high density double layer of polyamide nanoparticles. a) The electric field distribution and $b$ ) the resulting effect on the electric field values at $10 \mathrm{~nm}$. The electric field values are higher without the presence of nanoparticles.

The effects on the electric field and current density values were compared for single and double layers at low density. The results are in Figure 18. The values are almost identical at distances of 10 and $50 \mathrm{~nm}$ indicating that the size 
of the layer would not have an impact on the signal generated by an electrical biosensor.

a)

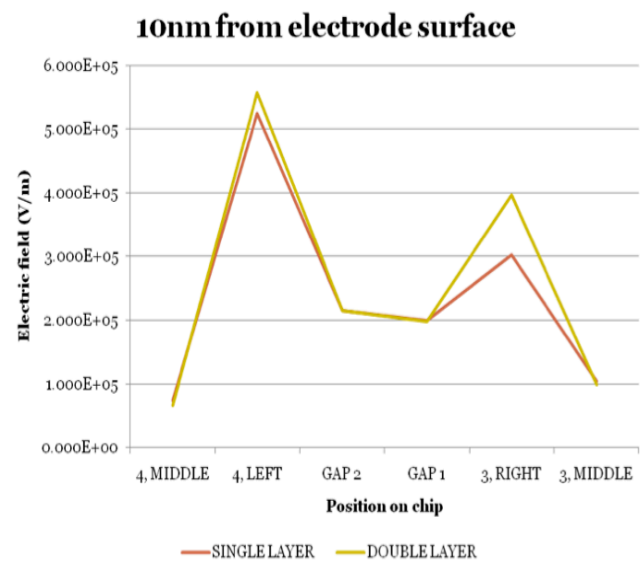

c)

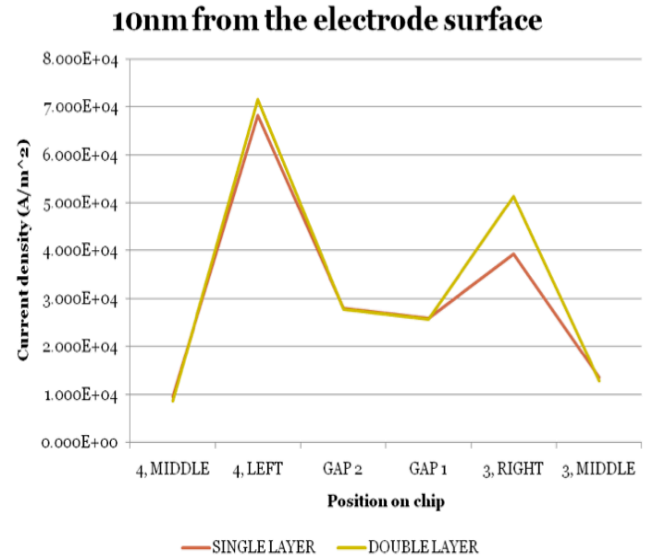

b)

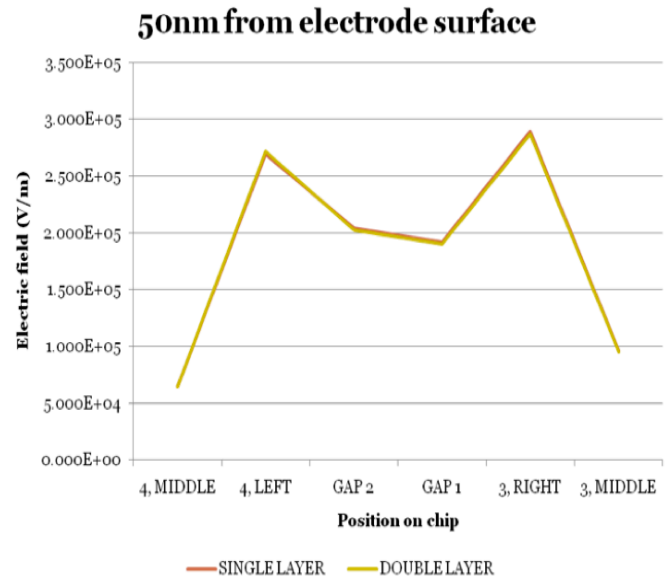

d)

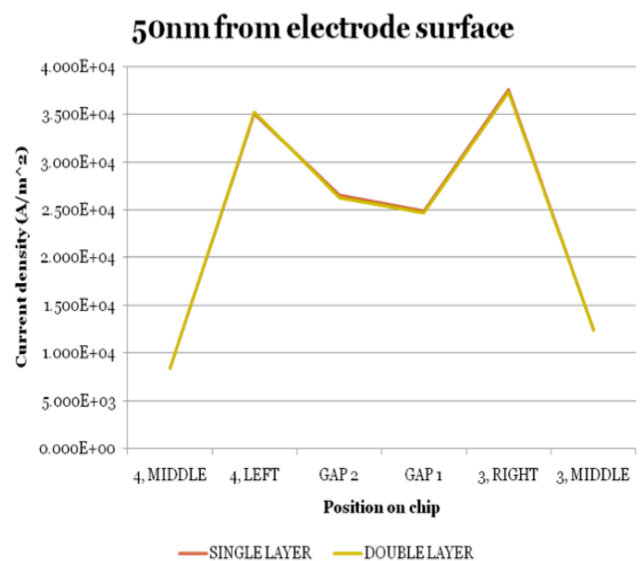

Fig 18. Comparison of effects from single and double layers of polyamide nanoparticles at low density. a,b,c,d) There is not an appreciable change in the electric field or current density values at any distance from the electrode surface.

The effects of the single and double nanoparticle layers at a high density were also compared as seen in Figure 19. Though data is only shown for $50 \mathrm{~nm}$ above the electrode surface, the data from 10nm above the electrode surface exhibits the same trend. At every point the single layer of nanoparticles creates a stronger electric field and current density than the double layer. This is a departure from the behavior observed for the single and double layers at low 
densities. At the lower density, the size of the layer has little to no effect on the electric field and current density.

a)

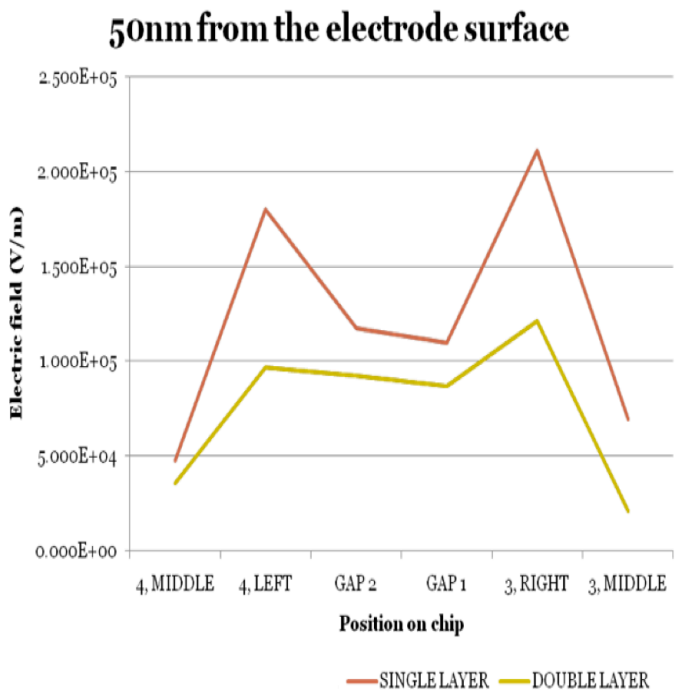

b)

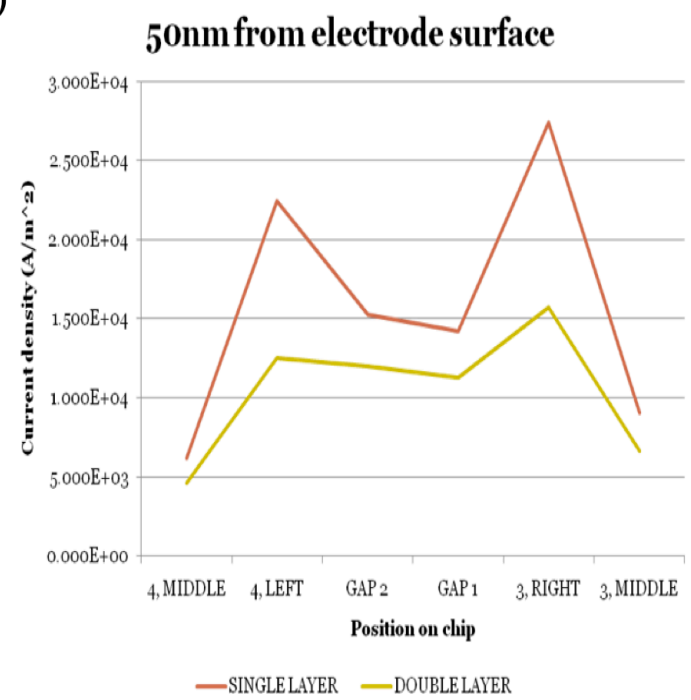

Fig 19. Comparison of effects from single and double layers of polyamide nanoparticles at high density. a,b) The electric field and current density values are higher for a single layer of nanoparticles.

It was necessary to determine if a low density or high density layer, regardless if the nanoparticles are in single or double layer, has a greater impact on the electric field and current density values. Figure 20 compares these values at a distance of $50 \mathrm{~nm}$ from the electrode surface. Though not shown, the data obtained at $10 \mathrm{~nm}$ from the electrode surface exhibited the same trend. At every position a low density layer of nanoparticles has a greater effect than a high density layer. 
a)

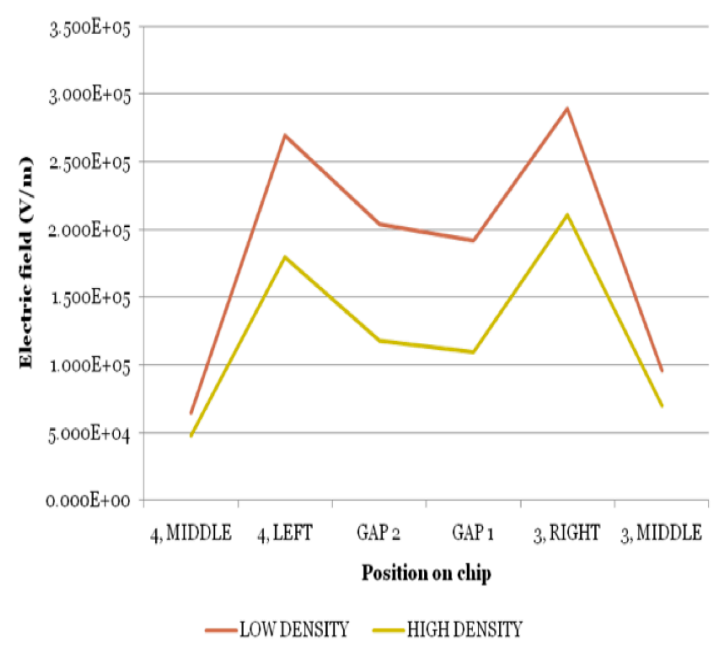

b)

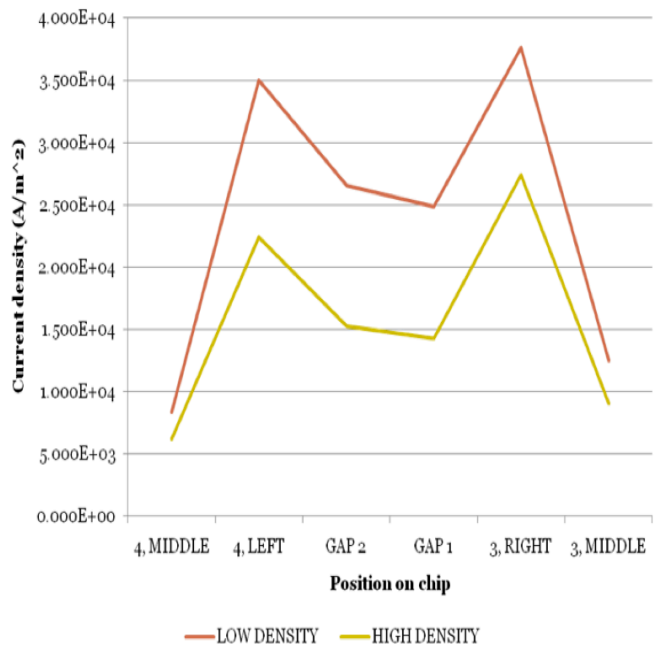

Fig 20. Comparison of high and low density polyamide nanoparticles. a,b) The electric field and current density values are greater for a low density of nanoparticles.

Many models were created to evaluate the effect of nanoparticles on the electrical biosensor. From the observed effect on the electric field and current density values, it was determined that polyamide nanoparticles $20 \mathrm{~nm}$ in diameter are not capable of consistently enhancing the signal generated by the biosensor. Should these nanoparticles be used, the best use of them should be in a single layer at a low density.

2.6 Indirect confirmation. Based on the results of the computational models created using Ansoft, it was clear that some small modifications to the basic electrode array design would greatly enhance the sensors sensitivity.

The modification that would have the largest impact was decreasing the gap between the interdigitated electrodes. IDEAs were fabricated using electron beam lithography for gap distances of 200, 300, 400, and $500 \mathrm{~nm}$. Figure 21 
represents the sensitivity of the fabricated devices. D2 (width of 400nm, gap of 200nm) was the most sensitive and D6 (width of $1500 \mathrm{~nm}$, gap of 500nm) was the least sensitive. D3 was fabricated with a width of $400 \mathrm{~nm}$ and a gap of $400 \mathrm{~nm}$, making it comparable to D2. D4 (width of 700nm, gap of 200nm) and D5 (width of $700 \mathrm{~nm}$, gap of $300 \mathrm{~nm}$ ) were comparable to each other. In creating two devices with the same width and different gap sizes it was possible to directly compare the effect of the gap size. D6 was the device most closely resembling the dimensions used in the IDEs fabricated by SHARP.

$R_{\mathrm{ct}}$ values for each device were obtained from Nyquist plots created from the sensors' impedance response under different concentrations of a redox probe. The Nyquist plots were fitted with Randles circuit using Z-view. These Rct values were plotted for each concentration for each device. A linear fit was obtained for each device's response. The slope of this line was divided by the area of the device. The resulting value was the aerial sensitivity of the device.

D2 had the highest aerial sensitivity value. It had a higher sensitivity than D3 which had the same width as D2 but twice the gap distance of D2 proving that a smaller gap size is key to more sensitive detection. The same result was seen for devices D4 and D5. Those devices had the same width but D4 had a smaller gap size by $100 \mathrm{~nm}$ and it resulted in a higher aerial sensitivity value for D4 than the value for D5. D6 was the least sensitive device and it had the largest gap size at $500 \mathrm{~nm}$. The width of D6 was the same as the IDEs fabricated by SHARP but its gap size was $300 \mathrm{~nm}$ less than the SHARP IDEs. This implies that the SHARP 
IDEs are less sensitive than any of the devices fabricated to verify the modeling results from section 2.2.

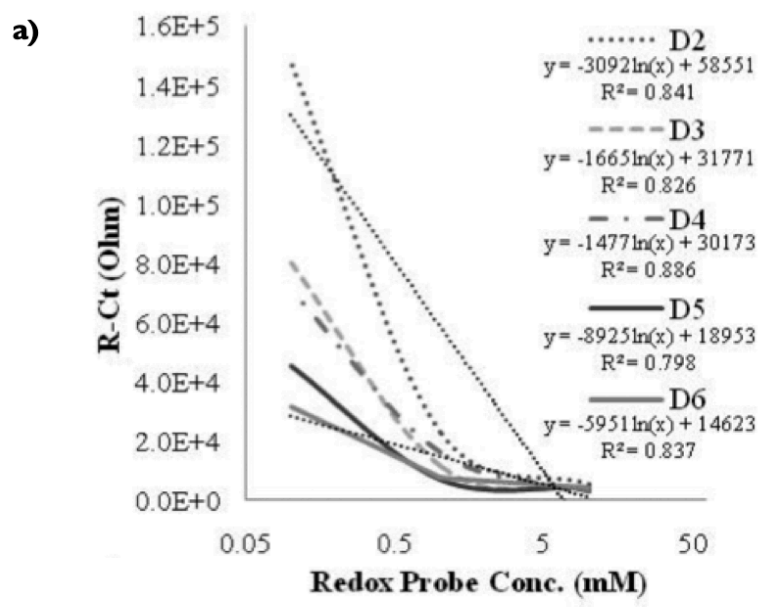

b)

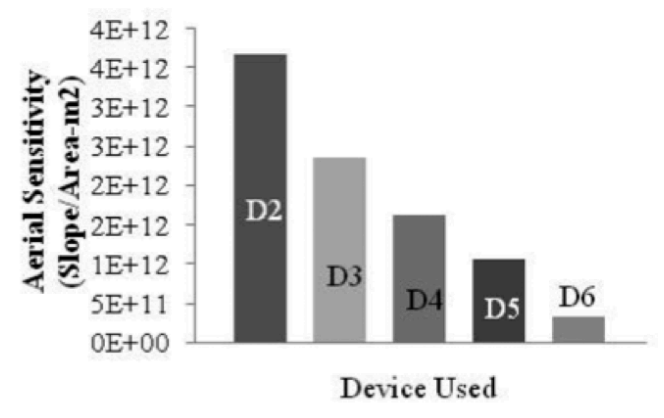

Fig 21. Determination of sensitivity of the devices fabricated through e-beam lithography. (a) RcT values plotted against the redox probe concentrations. For all devices, the slopes of the curves were determined from the best fit equations (shown as a straight lines only for devices D2 and D6 for clarity) for each curve (shown in the legend) and is termed device sensitivity. (b) Relative aerial sensitivities (slope per unit area) of different devices D2-D6. D2 has the highest aerial sensitivity value and D6 has the lowest aerial sensitivity value. 


\section{Chapter 3:}

Biosensor functionalization and immunosensor development 
3.1 IDEA chip functionalization procedure. In order for the binding event to occur between the target and the probe molecule an adequate sensing layer had to be developed on the electrodes on the IDEA chip. Figure 22 is a diagram of the functionalization process.

The first step consisted of covering the IDEs with a self-assembled monolayer (SAM) of molecules. This was achieved by submerging the chips in a SAM solution of $100 \mathrm{mM}$ cysteamine hydrochloride (Sigma Aldrich, St. Louis, MO) in $95 \%$ ethanol overnight.

Following this step, the chips were washed in a copious amount of $95 \%$ ethanol three to five times for five minutes at a time, followed by a 10 minute wash in DI water. Next, the chips were incubated on an orbital shaker in a $10 \mathrm{mM}$ solution of triethylamine (Sigma Aldrich, St. Louis, MO) in DI water for an hour. Subsequently, they were washed in copious amounts of DI water, three to five times for five minutes each time. The SAM layer was then activated by placing the chips in a $2.5 \%$ aqueous solution of glutraldehyde (Fisher Scientific, Fair Lawn, NJ) in PBS for two hours on an orbital shaker. The chips were then washed in a copious amount of $0.1 \mathrm{M}$ carbonate-bicarbonate buffer in DI water three to five times for five minutes each time. Subsequent to their washing, the chips were dried under nitrogen stream.

The next step was to anchor the probe molecule. A solution containing the probe in carbonate/bicarbonate buffer was placed on the electrodes and allowed to evaporate overnight. Using the glutraldehyde linker, the probes were 
immobilized onto the chip. Following this evaporation procedure, any unbound protein was washed away in three to five washings of five minutes apiece using the carbonate-bicarbonate buffer previously mentioned. During the next step, the unreacted aldehyde groups on the SAM were neutralized, and thus the conjugation reaction stopped, when the chips were placed in a $5 \%$ solution of ethanolamine (Sigma Aldrich, St. Louis, MO) in 0.1 M carbonate-bicarbonate buffer for one hour. Again, the chips were placed on an orbital shaker during this incubation period. After this step of the process is completed, the chips were washed with PBS containing $0.05 \%$ Tween 20 (PBST) following the same procedure as previously mentioned.

Finally, the nonspecific binding sites, or the areas on the electrodes that did not have a probe molecule immobilized onto them but were still available for binding activity, were blocked using a 1\% solution of polyvinylpyrrolidone (PVP), a non-protein polymer,(Sigma Aldrich, St. Louis, MO) in PBST. The chips were placed on an orbital shaker in this blocking solution for an hour. PVP lends itself to being an ideal blocking solution as it has thin layer formation, aqueous solubility, and biocompatibility. 


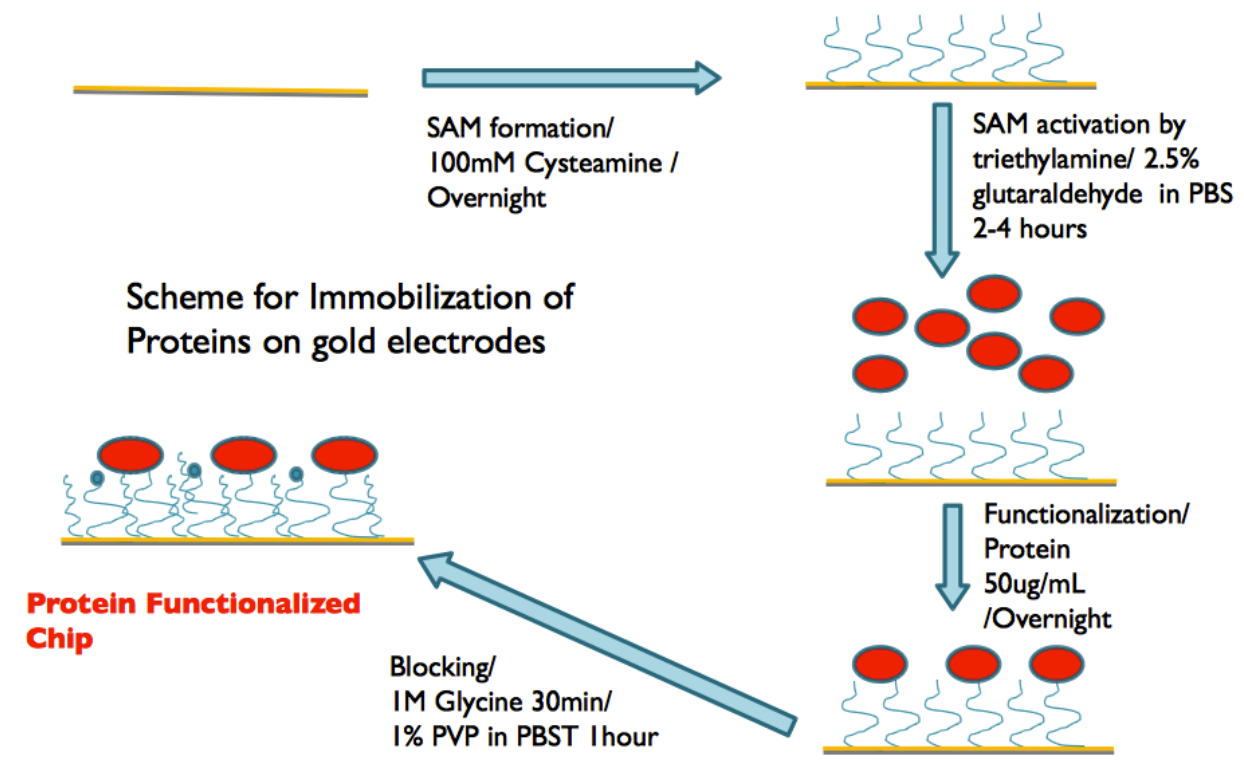

Fig 22. Overview of functionalization process. A SAM is developed on the gold electrodes and activated using triethylamine and glutraldehyde. The capture protein is attached to the SAM and the nonspecific binding sites are blocked using 1\% PVP. Image is not to scale.

At this point, the functionalization procedure was complete. Any chips not immediately used after this final step were stored in PBST at $4^{\circ} \mathrm{C}$ where they remained viable for 24-48 hours.

3.2 EIS measurements. The impedance measurements were made using an impedance meter (HP Agilent 4294A). Using software that was written to control the impedance meter, the electrical impedance spectroscopy (EIS) data was collected over a range of $50 \mathrm{~Hz}$ to $500 \mathrm{kHz}$ three times at a constant voltage of $25 \mathrm{mV}$ without DC bias when nonfaradaic data was being collected and with a $215 \mathrm{mV}$ DC bias when the faradaic data was being collected. These three sets of data were averaged at every frequency every time a new set of data was taken on an electrode. 
To begin the experiment, the chip was placed on a clean stage. A microfluidic channel made of Plexiglas was clamped on the chip using a gasket made of PDMS for easy handling of solutions and effective shielding of connectors from the measurement chamber. Two tubes attached to the Plexiglas channel allowed the fluid to enter and exit the channel and kept the fluid localized to the functionalized electrodes. Data was then collected by a probe station connected to the impedance analyzer. Figure 23 demonstrates the placement of the chip, gasket, and channel on the stage.

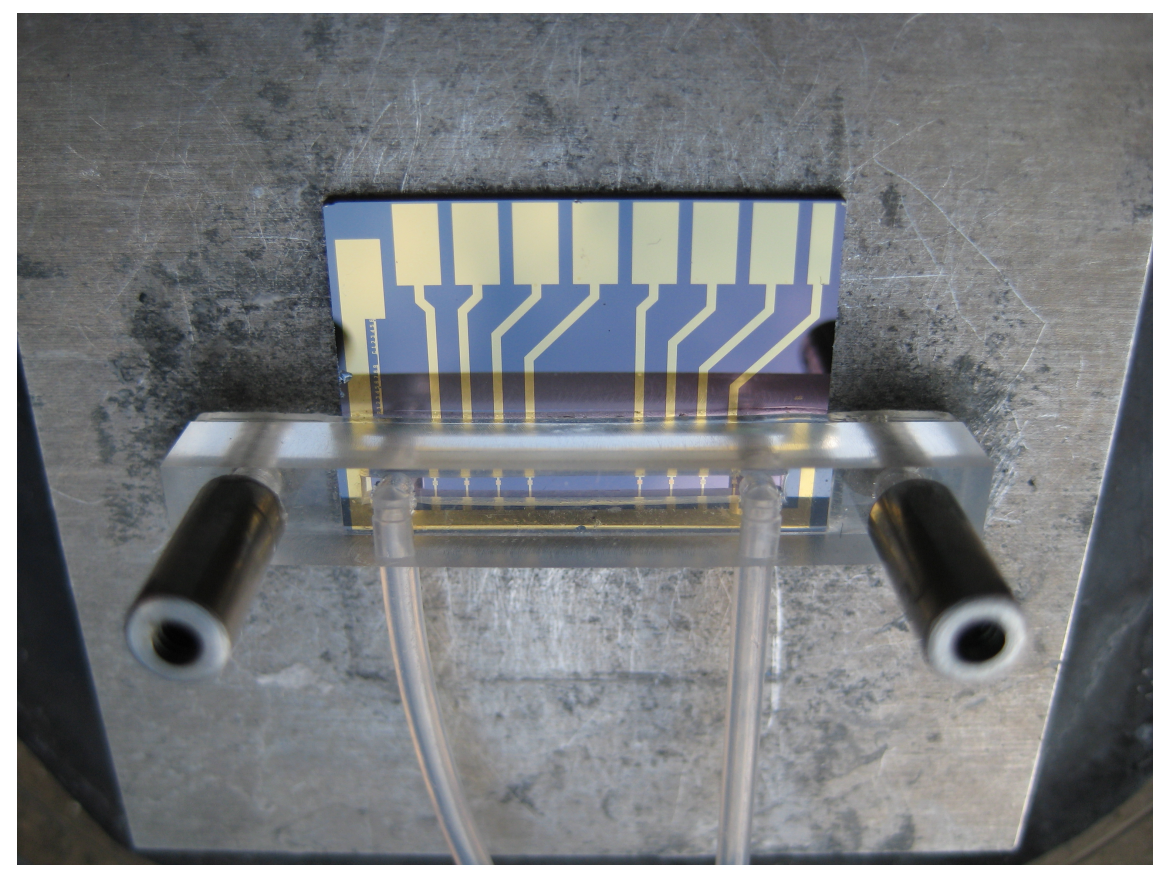

Fig 23. IDEA chip placed on a clean stage. The PDMS gasket and plexiglass channel are securely placed over the electrodes on the IDEA. The tube leads in and out of the channel allow for the liquid sample to be injected into the area directly surrounding the electrodes.

The EIS data was taken in PBST buffer solution. Data was collected before the beginning of the experiment, during the experiment as the sensor was 
incubated with varying concentrations of the target antigen or antigens for ten minutes, and after the channel on the chip was flushed through with a regenerating buffer solution of pH 2.00 from La-Mar-Ka (Baton Rouge, Louisiana). This buffer removed any bound molecules from the sensing layer. The three sweeps of data were averaged to form one set of data that contained the average impedance, including both its real and imaginary parts, and phase angle. The data was analyzed using Open Office Spreadsheet.

In this research, two methods of data analysis were used. They are both outlined in section 1.3, but they will be briefly outlined again below. One of the ways the data was analyzed was to directly compare the impedance measurements obtained after every set of data collection. One frequency was chosen, generally one between $50 \mathrm{~Hz}$ and $5 \mathrm{kHz}$, and the impedance measurement that resulted from one concentration binding to the sensing layer at that frequency is compared to the other impedance measurements obtained from the other concentrations at the same frequency. This resulted in a concentration dependent response.

A second way of analyzing the EIS data was to develop and analyze Nyquist plots. Nyquist plots were obtained using the real and imaginary components of the impedance. The data plots from the native electrode, after SAM layer formation, electrode with the capture antibodies, and electrode after antigen binding activities between the biolayer and the target molecule all serve to show that the biomolecular mass accumulation, at the electrode surface, was 
increasing with each subsequent step in the functionalization and experimental procedure. The data obtained was fitted with a Randels equivalent circuit using Zview software commercially available from Scribner Associates (Southern Pines, North Carolina), and the numerical values obtained for the electron transfer resistance and double layer capacitance were compared.

3.3 CA-125 biosensor. A biosensor, created using the procedure described in section 3.1, was developed for the detection of ovarian cancer biomarker CA-125. EIS was used to measure the binding between CA-125 and the sensing layer on the device.

Monoclonal antibodies for CA-125 were purchased from US Biological (Swampscott, MA). antiCA-125 at a concentration of $50 \mu \mathrm{g} / \mathrm{mL}$ in carbonate/ bicarbonate buffer were anchored onto the chip by placing the solution over the electrodes and allowing it to evaporate overnight. The rest of the functionalization procedure was executed exactly as described in section 3.1.

After the sensing layer was activated and the nonspecific binding sites blocked, the chip was exposed to PBST spiked with CA-125 at concentrations of $10,20,40$, and $80 \mathrm{U} / \mathrm{mL}$ to cover the clinically relevant concentrations. The cutoff concentration of CA-125 that indicates disease is $35 \mathrm{U} / \mathrm{mL}$. CA-125 was injected into the channel and allowed ten minutes to bind to the sensing layer before the excess, unbound CA-125 was washed out of the channel using PBST and the EIS data collected. 
For these experiments, the change in impedance due to antigen binding was compared at different concentrations. Baseline data was determined using the average impedance values before any binding of the target protein to the sensing layer and after regeneration of the chip when the bound proteins are removed from the sensing layer.

The sensor was also used to detect CA-125 concentration in 5\% rabbit serum. The serum solution was created in PBST with $5 \%$ of the volume being rabbit serum. Again, the serum solution was spiked with CA-125 at concentrations of $10,20,40$, and $80 \mathrm{U} / \mathrm{mL}$. The data was collected and analyzed in the same way as the measurements made in spiked buffer solution.

3.4 CEA biosensor. Following the procedure in section 3.1, monoclonal antibodies to CEA, also purchased from US Biological, were anchored onto the IDEA chip at a concentration of $50 \mu \mathrm{g} / \mathrm{mL}$ by allowing the antibody solution to evaporate overnight. The sensing layer was activated and the nonspecific binding sites were blocked. EIS was used to make the device measurements.

The device was exposed to CEA in concentrations ranging from $1 \mathrm{pg} / \mathrm{mL}$ to $10 \mu \mathrm{g} / \mathrm{mL}$ in 10 fold concentration increments in PBST to cover a broad range. The cutoff for clinical diagnosis of disease is $10 \mathrm{ng} / \mathrm{mL}$ in serum. These concentrations were present in spiked buffer and in the same $5 \%$ serum solution described in section 3.3. In both spiked buffer and serum, CEA was given ten minutes to bind with the sensing layer before any unbound CEA was removed 
using PBST flushed through the channel and the EIS data collected. The data was obtained and analyzed as described in section 3.2

Three sets of data were collected on the sensors created for the detection of CA-125: the initial data before any binding to the sensing layer occurred, the binding data from the binding between the sensing layer and CA-125, and the final data that was a result of the low $\mathrm{pH}$ regeneration buffer removing any bound CA-125 from the sensing layer.

\subsection{Multiplexed biosensor for the detection of CA-125 and CEA. A biosensor} capable of detecting both CA-125 and CEA was developed following the basic protocol outlined in section 3.1. A PDMS gasket that effectively separated the eight interdigitated electrodes into two groups of four was placed on the IDEA chip. Four of these electrodes had a solution containing $50 \mu \mathrm{g} / \mathrm{mL}$ of monoclonal CA-125 antibodies placed over them and the remaining four electrodes had a 50 $\mu \mathrm{g} / \mathrm{mL}$ solution of monoclonal CEA antibodies placed over them. Both solutions were allowed to evaporate onto the chips overnight. Figure 24 is a diagram of the functionalization of the multiplexed chip. 
a)
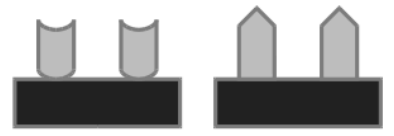

d)

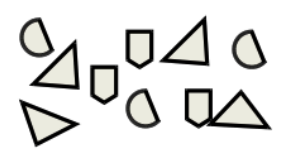

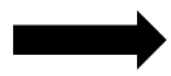

b)

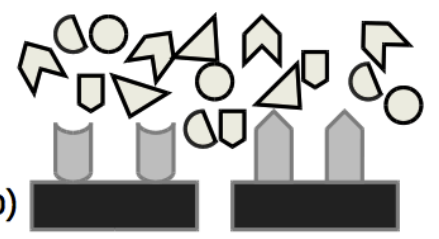

c)

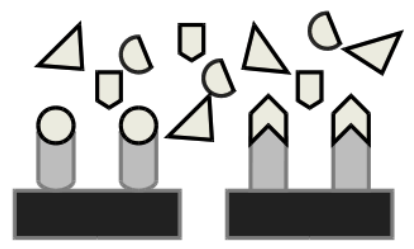

Fig 24. Overview of functionalized multiplexed chip. a) Electrodes are functionalized for the specific capture of different antigens. b) A serum sample containing target antigens for both electrodes is exposed to the sensing layers on the electrodes, c) the target antigen for each electrode binds to the electrodes, and d) the unbound antigens are washed away. Image is not to scale.

Spiked PBST containing 10, 20, 40 and $80 \mathrm{U} / \mathrm{mL}$ of CA-125 and 1, 10, and $100 \mathrm{ng} / \mathrm{mL}$ of CEA was detected by the multiplexed sensor. The same $5 \%$ rabbit serum solution as described in section 3.3 was also spiked with CA-125 concentrations of 20 and $40 \mathrm{U} / \mathrm{mL}$ and CEA concentrations of 10 and $100 \mathrm{ng} / \mathrm{mL}$ and detected by the sensor. The data was again analyzed using the methods detailed in section 3.2 .

In all cases, the target antigen was allowed ten minutes to bind to the sensing layer before any unbound protein was washed from the channel with PBST and EIS data was collected. A total of three sets of data were collected from these biosensors: the initial data before any binding to the sensing layer occurred, the binding data that resulted from the attachment of CA-125 or CEA to 
the sensing layer, and the final data that was collected after the regeneration buffer removed the bound protein from the sensing layer.

The multiplexed chip had a unique advantage built into its design. When binding data for one of the proteins was collected, it was collected on every electrode regardless of whether the electrodes were functionalized for its detection or not. In doing this, a built-in mechanism to check the specificity of the functionalized electrodes was created. Figure 25 is a diagram representing the binding between the electrodes and their specified antigen targets.

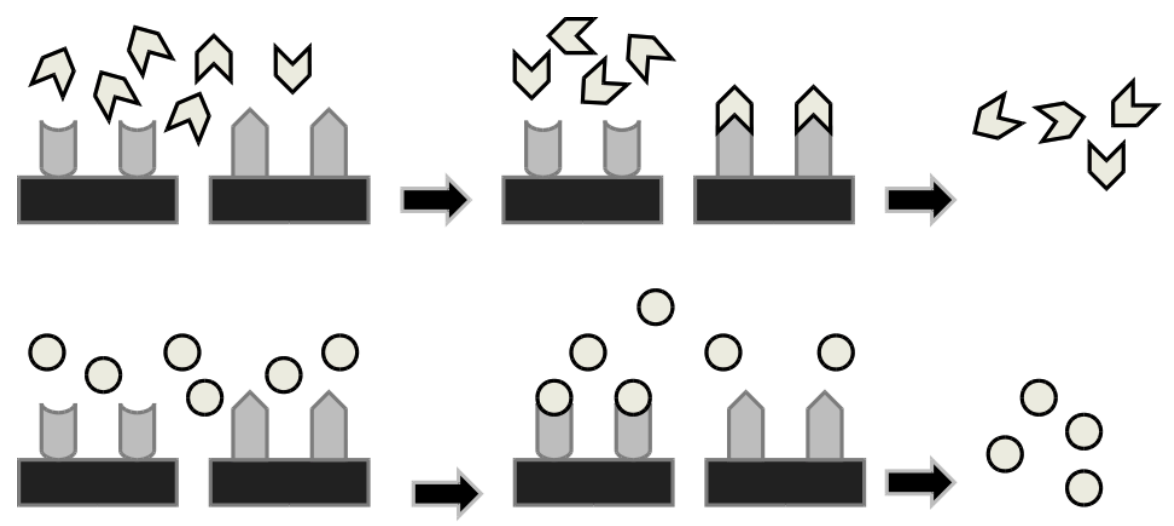

Fig 25. Diagram representing electrodes functionalized for the detection of different targets. A sample containing either CA-125 or CEA was given time to bind to all the electrodes whether they were specified for its detection or not. After the unbound antigen was washed away EIS data was collected on all the electrodes. In doing this the specificity of the functionalized electrodes could be qualified. Image is not to scale.

For example, when CA-125 was captured by the sensor, the binding data collected from electrodes functionalized specifically for the detection of CA-125 was compared to the binding data collected from the electrodes specifically functionalized for the detection of CEA. This process was repeated during the 
detection of CEA. In doing this it was possible to determine the extent of the specificity exhibited by each set of electrodes for its target protein. Specific and nonspecific detection by each set of electrodes was quantified.

The multiplexed sensor offered a distinct advantage over the sensors developed for the detection of CA-125 and CEA alone. CA-125 and CEA alone are unsuitable for early detection of ovarian cancer because neither is always present at elevated levels in patients and is also indicated in other non-ovarian cancers. Also, neither is present at elevated level in all three types of ovarian cancer.

CA-125 and CEA are both effective at determining the effectiveness of treatment and detecting both simultaneously promises to give more sensitive feedback about the treatment course. By creating a multiplexed sensor, it is possible to more comprehensively detect ovarian cancer's fingerprint and produce fewer false positives for the disease. The thought is that if both CA-125 and CEA are detected at elevated levels in one patient sample the likelihood of correctly diagnosing ovarian cancer at an early stage will be increase.

3.6 He4 biosensor. There were two biosensors developed for the detection of He4 and they both differed from the sensors developed for the detection of CA-125 and CEA in several ways. The He4 sensors relied on labels whereas the CA-125 and CEA biosensors are label-free. One of the sensors utilized a secondary antibody as a label and the other sensor included a biotinylated 
secondary antibody and additional binding with streptavidin and horseradish peroxidase (HRP) and enzymatic activity by HRP with a substrate to enhance the biosensor's signal.

The data gathered from the He4 sensors is also different from the data obtained from the CA-125 and CEA sensors in that it was taken in the presence of a redox probe. This is the faradaic EIS and for both of the He4 biosensors all data was collected in the presence of a redox buffer called PBSR $(2.5 \mathrm{mM}$ potassium ferrocyanide $\left(\mathrm{K}_{4}\left[\mathrm{Fe}(\mathrm{CN})_{6}\right] \cdot 3 \mathrm{H}_{2} \mathrm{O}\right), 2.5 \mathrm{mM}$ potassium ferricyanide $\left(\mathrm{K}_{3}\right.$ $\left[\mathrm{Fe}(\mathrm{CN})_{6}\right], 0.1 \mathrm{M} \mathrm{KCl}$, and PBS). This redox buffer is oxidized and reduced by electron transfer from the metal electrodes. To keep the redox species from being depleted the data was gathered under a DC bias condition of $215 \mathrm{mV}$. He4 and two types of monoclonal antibodies were obtained from Abnova (Taipei City, Taiwan). A $25 \mu \mathrm{g} / \mathrm{mL}$ solution of the monoclonal antibodies deemed suitable for capture by the manufacturer was evaporated onto the IDEA chip as in section 3.3. The IDEA chip was functionalized according to the protocol in section 3.1.

The He4 biosensor was developed to detect an immunocomplex composed of $\mathrm{He} 4$ antigen and a secondary antibody that are already bound together. A variable concentration of $\mathrm{He} 4$ antigen was combined, in PBST, with a secondary detection antibody, as determined by the manufacturer, the concentration of which was held steady at $10 \mu \mathrm{g} / \mathrm{mL}$. The antigen and the antibodies were allowed ten minutes to bind before being injected into the 
channel over the IDEA chip. The immunocomplexes were created using antigen concentrations of $1.5,3.125,6.25,12.5,25,50$, and $100 \mathrm{ng} / \mathrm{mL}$, all of which are in the currently determined diseased range for ovarian cancer.

The immunocomplex was given ten minutes to bind to the sensing layer before any unbound material was washed from the channel with PBST/PBSR and the data was collected.

In using the secondary antibody it was possible to generate a larger signal from the biosensor as presumably there would be more material bound to the sensing layer. It offers the advantages of amplifying the signal without altering the functional and binding abilities like a label can do. By holding the concentration of the secondary antibody steady and altering the antigen concentration it was still possible to directly correlate the signal generated by the sensor to the concentration of $\mathrm{He} 4$ bound to the sensing layer.

A total of three sets of EIS data were collected for these biosensors: the initial data before any attachment to the sensing layer, the binding data when the immunocomplex had bound to the layer, and the final data after the bound immunocomplexes were removed from the sensing layer by the low $\mathrm{pH}$ regeneration buffer.

Another biosensor for the detection of $\mathrm{He} 4$ using the same premise of attaching more material to the sensing layer was also developed. In this sensor, the monoclonal antibody suitable for capture, again at $25 \mu \mathrm{g} / \mathrm{mL}$, was evaporated onto the electrodes overnight and functionalized following the protocol in section 
3.1. Immunocomplexes containing $1.5,3.125,6.25,12.5,50$, and $100 \mathrm{ng} / \mathrm{mL}$ of He4 combined with a biotinylated secondary antibody at a concentration of 10 $\mu \mathrm{g} / \mathrm{mL}$ were created after allowing the two components to bind for ten minutes. Figure 26 is a diagram of the biolayer developed on the sensing layer.

The biotinylated immunocomplexes were given ten minutes to bind to the sensing layer before the unbound complexes were washed away with PBST/ PBSR and the data collected.

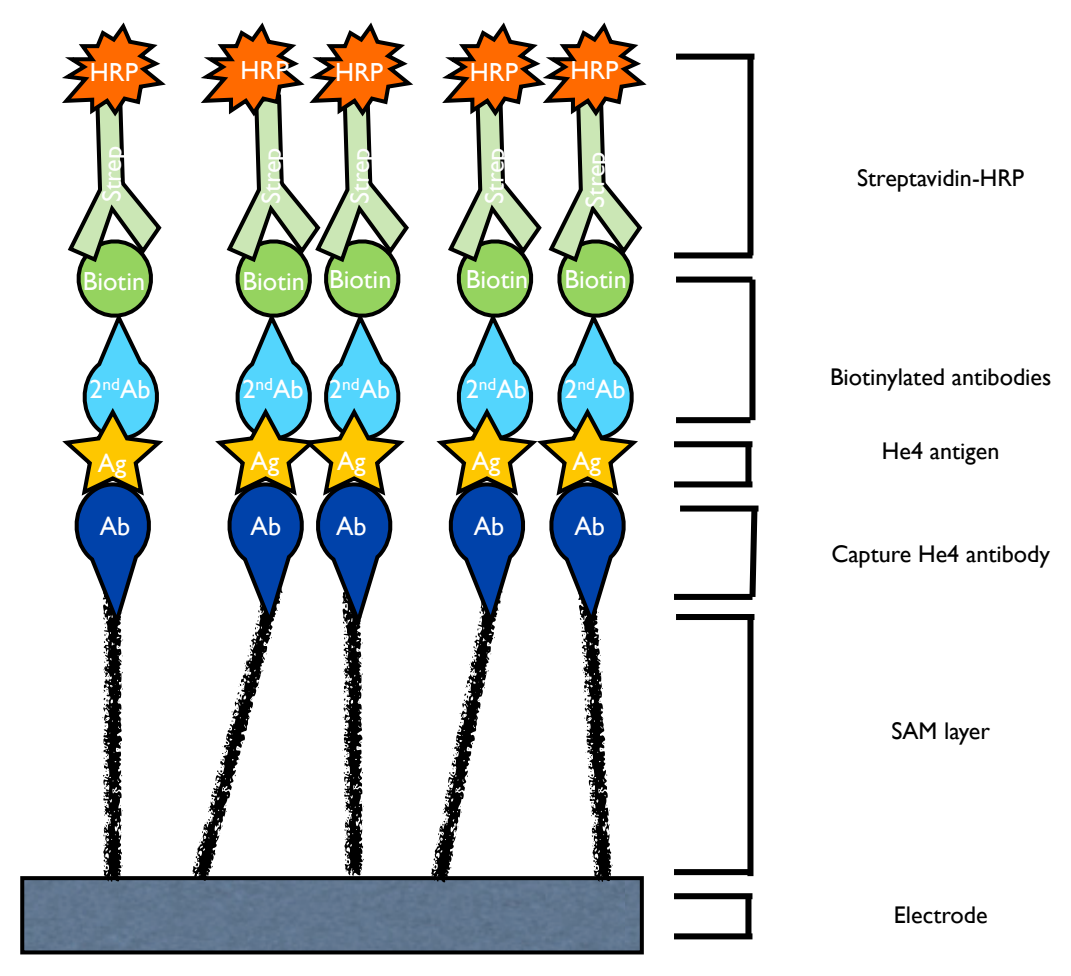

Fig 26. Diagram of the biolayer developed on the electrode of the He4 sensor. On top of the sensing layer, which includes the SAM and capture antibody, the He4 antigen, already bound to the biotinylated secondary antibody, is bound. Next the streptavidin-HRP conjugate is introduced and it binds to the biotin on the secondary antibody, leaving the HRP ready for activity. Image is not to scale. 
Biotinylated secondary antibodies were obtained from the University of Washington (Seattle, WA). Biotinylation is achieved by the addition of several biotin molecules at the primary amine sites of an antibody. Streptavidin is a natural binding partner of biotin; the dissociation constant of streptavidin-biotin binding is about $10^{14} \mathrm{~mol} / \mathrm{L}$, making it one of the strongest non-covalent interactions in nature [52]. The streptavidin-biotin complex is resistant to heat, $\mathrm{pH}$ change, and interactions with solvents and denaturing agents. Because of this, it is commonly used in biotechnology applications.

The biotinylation of the secondary antibody allowed for the formation of yet another binding layer, this one achieved by the incubation of $1 \mu \mathrm{g} / \mathrm{mL}$ of streptavidin conjugated with horseradish peroxidase (US Biological, Swampscott, MA) (HRP) over a period of ten minutes. After that, excess streptavidin-HRP was washed from the channel using PBST/PBSR and data was collected. HRP is an enzyme that is commonly used in biochemical situations that require signal amplification. When in the presence of a substrate containing hydrogen peroxide as the oxidizing agent, HRP produces a visible color change, in the case of ELISA, or a precipitable product, in the case of membranes, directly correlated the amount of HRP present.

The streptavidin bound to the biotin and the HRP was available for activity. A TMB substrate specific for interaction with HRP, purchased from Sigma Aldrich (St. Louis, MO) was injected into the channel on the chip and given five minutes to react with the HRP. This particular substrate is for use in situations that require 
the formation of a precipitate. After five minutes EIS data was collected without washing the channel.

A total of four sets of EIS data was collected from these biosensors: the initial data before the incubation of any proteins, the first set of binding data between the biotinylated immunocomplex and the sensing layer, the second set of binding data between the streptavidin-HRP and the biotinylated immunocomplex, and the third and final set of binding data that was a result of the formation of the precipitate from the HRP-substrate interaction. 


\section{Chapter 4:}

Aptamer selection and molecular cloning procedures 
4.1 Modified SELEX procedure for aptamer selection and identification. The traditional method of aptamer selection, SELEX, relies on radioactive labels to identify the sequence of the binding strand of DNA. As such, this method calls for an isotope laboratory to carry out the experiment, as well as an overall elevated level of caution and cost.

A slight modification to the procedure eliminates any need for such restrictions. By simply amplifying the binding sequence using polymerase chain reaction (PCR) described in the following section 4.2, a fluorescent label can be introduced that allows for identification of the binding sequence [53]. This label is incorporated into one of the primers used to run the PCR. The other primer used in the PCR process that binds to the strand complementary to the binding sequence also contains a label. This label is a sequence of 18 adenine nucleotides, called a poly $\mathrm{A}$, that cause that product to be 18 nucleotides longer than the binding strand. In this way we have two different sized PCR products with the binding sequences easily identifiable by their fluorescent label and the non-binding but complementary strand identifiable by its length as seen in Figure 27.

The process begins by immobilizing the target protein on a nitrocellulose membrane. An overview of the entire process can be seen in Figure 28. The target is whatever protein for which the researcher is trying to find an aptamer. After immobilization, the membrane is washed in a buffer conducive to binding. In the same buffer a quantity of a random DNA library, containing about $4^{60}$ different 
sequences with known ends, is introduced to the membrane. Binding is allowed to continue for two hours. After two hours, any remaining unbound sequences are removed and any sequences that bind to the target are eluted from the membrane. These sequences are then amplified using PCR with the aforementioned modified primers.

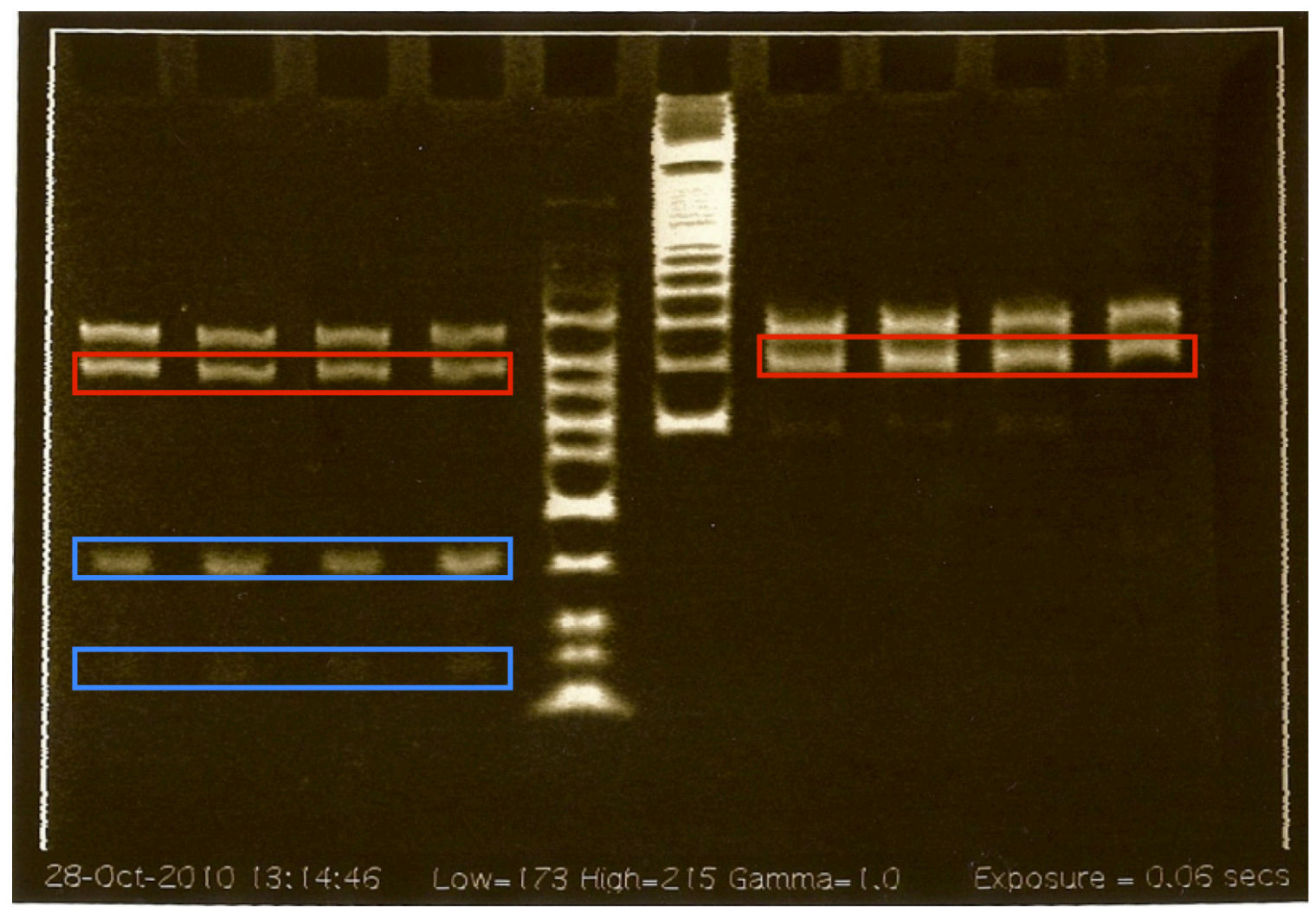

Fig 27. The double stranded PCR product from the modified SELEX procedure after being run through a denaturing polyacrylamide gel. The shorter strand, identified by the red box and its relative position to the two DNA ladders, is the known binding sequence and it's longer complementary product is located above it. The binding strand is excised from the gel, the DNA eluted from it, and the DNA sequences contained within used in the next SELEX round. The blue boxes indicate the primers the remaining primers that have not been used by the PCR reaction. The top blue box contains the longer primer with the poly $A$ and the bottom blue box contains the smaller primer with the FITC label. As measured against the DNA ladder, the longer primer is twice the length of the FITC labeled primer. This gel has been stained with ethidium bromide so that both PCR products are visible. 
After amplification, the double stranded PCR products are separated and run through a denaturing polyacrylamide gel. Gel electrophoresis is described below in section 4.3. Due to the fluorescent label, the binding sequence is easily identified in the gel using UV illumination. The band of binding sequences is excised from the gel with the longer complementary strand left behind. The binding DNA is eluted from the gel and those binding sequences are used in the next round of selection against the membrane containing the immobilized target. 
a)

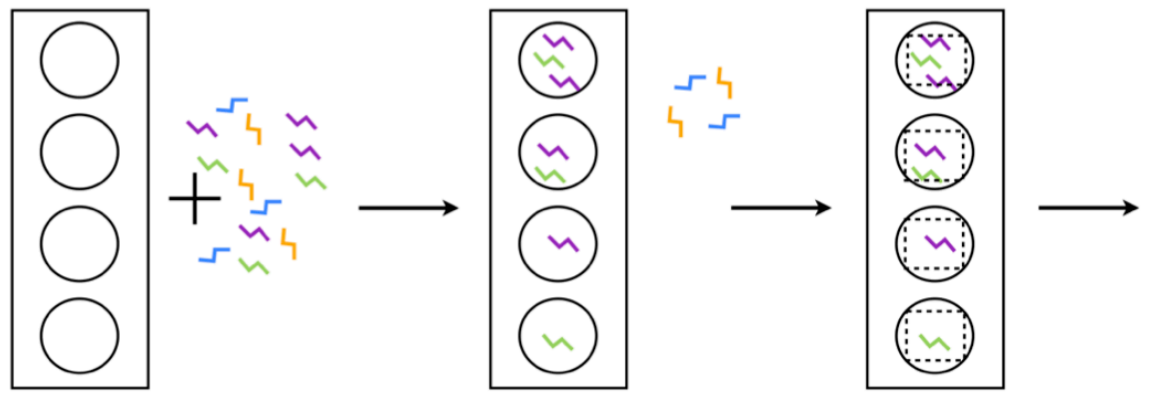

b)

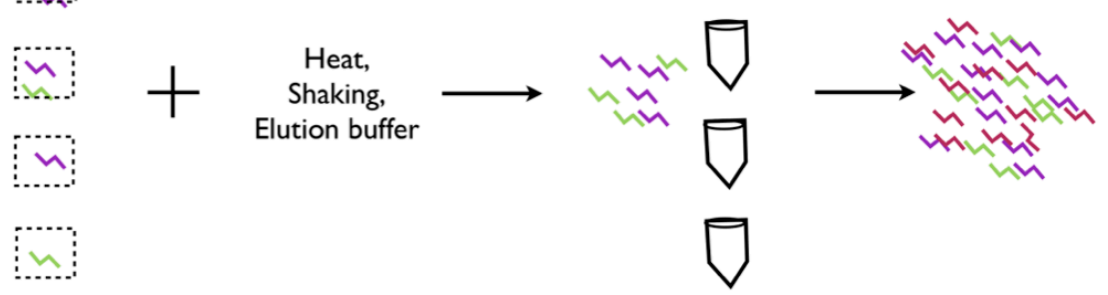

c)
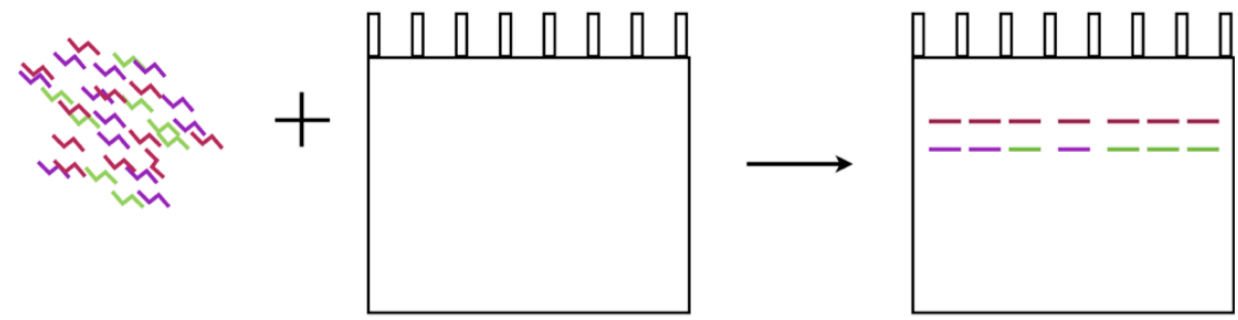

d)
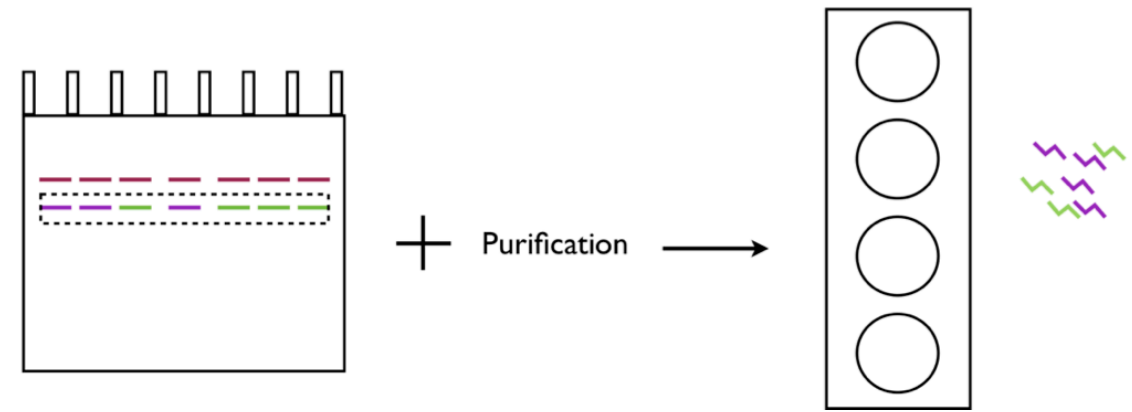

Fig 28. Overview of SELEX process. a) A nitrocellulose membrane spotted with CA-125 is exposed to a random ssDNA library. Some aptamers bind to the CA-125 on the membrane and the ones that do not bind are washed away. b) The centers of the membrane spots containing the bound aptamers are excised and those aptamers removed from the CA-125. Those aptamers are run through a PCR to amplify them. c) The aptamer pool is run through a denaturing polyacrylamide gel and the PCR products are separated with the longer, complimentary strands to the aptamers (red) produced by the PCR located above the amplified binding strands (green and purple). d) The aptamers are excised from the gel, purified and isolated, and used in the next SELEX round where they are exposed to a nitrocellulose membrane spotted with CA-125. 
Typically several rounds of this selection process are carried out, with somewhere around ten being standard. It is also a good idea to carry out a few rounds of counter selection against a different target protein. The binding sequences collected after ten rounds of selection are collected and exposed to a different protein immobilized on a membrane. Several of these counter selection rounds are carried out with any sequences that bind to the counter selective target being removed from the pool of binding sequences. These specific binding sequences are then cloned with bacteria. The cloning process is described below in section 4.4. Colonies are picked and the DNA extracted from them and purified. Sequencing follows and, ideally, several different binding sequences are returned.

Once the sequences are known their binding capabilities must be explored. Attaching a fluorescent label to the different binding sequences is one method that allows for easy quantification in binding assays. By measuring the fluorescence that results from direct binding between the sequence and the target it is possible to evaluate the relative binding capabilities of the known sequences. Also using the fluorescence as a relative quantification tool, a competitive binding assay can be carried out using the binding sequence and the target protein's actual complementary protein. This assessment is an effective tool for comparing the binding of the DNA to the binding of the protein's natural binding partner. Dissociation constants can also be measured as a way to compare the binding ability of the different sequences. 
For this research, an aptamer specific to CA-125 was selected using this modified SELEX process. The goal was to replace the CA-125 monoclonal antibody used as the capture mechanism currently used in the electronic biosensor with the selected aptamer.

4.2 Polymerase chain reaction. Polymerase chain reaction, $\mathrm{PCR}$, is the best in vitro method to amplify DNA. While molecular cloning produces large quantities of amplified DNA, PCR is able to amplify DNA even when it is impure or present in very low quantities. PCR is characterized by a relatively simple three step process. First, the DNA templates are heated to denature the DNA so that all strands are separated. Next, the templates are cooled to allow hydrogen bonds to form between the primers and the ends of the template. Finally, the templates are heated to a high enough temperature that a DNA polymerase enzyme becomes active and adds nucleotides to the 3' end of the DNA, creating new, complete strands of DNA. This three step cycle is then repeated as many times as necessary, usually between 20 and 30 rounds. Because every cycle results in the doubling of the DNA strands, the addition of a few cycles results in a large increase in the amount of PCR products.

The key to running a PCR is to have DNA templates with known sequences at the 5' and 3' ends. By knowing these sequences, complimentary primers can be used to bind to the template and extend the sequence from the 5' end to the 3' end of the unknown part of the template, thus amplifying the DNA by 
creating new strands of it. The DNA polymerase is also an important part of the PCR process. Most enzymes are destroyed by high levels of heat therefore a heat-stable polymerase must be used.

The conditions for PCR must be determined for efficient and specific amplification. Generally the heating temperature is above $90^{\circ} \mathrm{C}$ to ensure complete denaturation of any double stranded DNA. The annealing temperature is unique to each sequence amplification; as it is the temperature at which the primers bind to the 5' and 3' ends, it is determined by the nucleic acid composition of the primers. The final elongation temperature at which the polymerase adds nucleotides to the forming sequence is usually $72^{\circ} \mathrm{C}$. This is the temperature at which most polymerases are active.

The PCR cycles at these temperatures are carried out in a short amount of time; 30 seconds to a minute is standard with longer sequences requiring more time at each step.

Finally, after all cycles are completed the PCR products go through a period of extension time at $72^{\circ} \mathrm{C}$ for ten minutes. The PCR products can then be stored at $-20^{\circ} \mathrm{C}$ until they are of further use.

4.3 Gel electrophoresis. Gel electrophoresis is a common molecular biology technique used to separate proteins and DNA based on their weight and charge. The gel acts a sieve of polymer fibers. The size of the pores within the fibers are directly related to the gel's chemical composition. As the gel electrophoresis 
experiments described in this dissertation were done using DNA samples, the discussion on the technique will be limited to its use in DNA applications.

DNA is negatively charged and as current is supplied to the gel, the DNA moves towards the positive terminal through the pores in the gel. Longer DNA sequences are more impeded by the fibers in the gel than shorter sequences and the shorter sequences migrate through the gel more quickly. This allows DNA sequences of different sizes to be effectively separated.

The two primary reagents used to create gels used in electrophoresis are agarose and polyacrylamide. Agarose gels are helpful in distinguishing between long DNA sequences and sequences that vary greatly in size. Agarose gels are run horizontally in TAE buffer containing $40 \mathrm{mM}$ Tris, $20 \mathrm{mM}$ acetic acid, and 1 mM EDTA. Polyacrylamide gels can be used to distinguish between DNA sequences only differing in size by a few nucleotides even if the sequences themselves are relatively short. Polyacrylamide gels are run vertically in TBE buffer containing $89 \mathrm{mM}$ Tris, $89 \mathrm{mM}$ boric acid, and $2 \mathrm{mM}$ EDTA. Polyacrylamide gels offer an advantage in that they can be made to be denaturing by the addition of a denaturing reagent such as urea or formamide. This denaturing property of the gels is what enables the identification of a denatured PCR product where the sequences differ in length by only a few nucleotides.

It is necessary to track the DNA sample's progress as it migrates through the gel so that the sample doesn't migrate completely out of the gel. One way to do this is to include dye in the buffer used to load the sample. Common choices 
include xylene cyanol, bromophenol blue, and orange $\mathrm{G}$. These dyes usually contain a small amount of glycerol to weigh the DNA sample down so that it stays in the well of the gel before the power supply is turned on and the sample begins to move through the gel. The dyes move more quickly through the gel than the sample; as the dye reaches the end of the gel, the sample is still safely inside the gel.

Base pair ladders and mass ladders can be used in conjunction with gel electrophoresis to quantify the size or weight of the DNA sample that has under gone electrophoresis. The ladder is loaded into one of the wells in the gel and run through the gel parallel to the sample. After the gel has finished running, the sample bands and the bands comprising the ladder are visualized and compared. By doing this the size and the weight of the sample can be verified. Identifying DNA in a gel usually requires staining unless a visible label is incorporated into the PCR product. Ethidium bromide is commonly used to stain gels containing DNA. Ethidium bromide intercalates with double stranded or single stranded DNA and fluoresces orange when exposed to UV light. The results of ethidium bromide staining can be seen in Figure 27. However, ethidium bromide is thought to be mutagenic because it inserts itself between two strands of DNA and is considered a hazardous material. There are other non-mutagenic stains that have become available recently, but in this research staining was not used before the DNA sample was removed from the gel. Using the fluorescent label in the PCR products, the aptamer was identified and excised easily using a 
UV light. The gel was stained afterwards with ethidium bromide to ensure that the PCR product had indeed been double stranded and of the correct size and that the remaining, longer strand was still in the gel. If the aptamer had been stained with ethidium bromide before its excision from the gel, contamination of equipment and reagents would have resulted.

Gel electrophoresis is a handy tool in isolating DNA. DNA can be removed and recovered from an agarose, polyacrylamide, and denaturing polyacrylamide gel without altering the DNA in any way. A solution to solubilize the gel surrounding the DNA and a method for purification are all that are required to do so. Many biological supply companies sell kits for such a purpose.

4.4 Molecular cloning. Molecular cloning is the best way to reproduce large quantities of a chosen DNA sequence. Escherichia coli is a commonly used bacteria for molecular cloning. The bacteria contains a plasmid which is a small, circular DNA molecule that self-replicates. The plasmid DNA is not related to the bacterial chromosome which is located elsewhere in the bacteria. The plasmid can be altered and made to produce copies of a chosen DNA sequence.

The selected DNA sequence is inserted into the plasmid DNA forming a recombinant DNA plasmid. This plasmid is then inserted into a bacterium. As the bacterium clones itself, the chosen DNA sequence is cloned along with it producing a colony of bacteria containing the chosen DNA insert. This plasmid DNA can then be extracted from the bacteria colony by lysing the bacteria and 
collecting and purifying the cell remnants. If the DNA sequence that is used in the insert is responsible for protein expression, the protein is also produced in the cloned bacteria colony and can be extracted just as the sequence is. Many biological suppliers produce kits intended for such purposes.

\subsection{Modified SELEX procedure. A random ssDNA library containing} approximately $4^{60}$ sequences was acquired from Integrated DNA Technologies (Coralville, IA). Each random sequence consisted of a central section composed of 60 nucleotides, which were random, flanked on both sides by a known string of 18 nucleotides. Knowing this string was key as this is where the primers used later on in PCR would have to bind to duplicate the binding sequences. The basic design of the library sequences and PCR primers is seen in Figure 29.

Random DNA library sequence 5'-ATACCAGCTTATTCAATT 60N AGATAGTAAGTGCAATCT-3'

$$
\text { Modified primer I 5'-FITCIATACCAGCTATTCAATT-3' }
$$

Modified primer 2

$$
\text { 5'-AAAAAAAAAAAAAAAAAA/AGATTGCACTTACTATCT-3' }
$$

Fig 29. Sequence diagrams of the aptamer libraries and primer used in PCR. The library contains $4^{60}$ different sequences flanked on both sides by known sequences of 18 nucleotides. The modified primer 1 contains a FITC label making it easy to find with a UV source. Modified primer 2 contains a poly A label of 18 adenosine nucleotides, making its PCR products longer than the products created by modified primer 1 .

$10 \mu \mathrm{g}$ of CA-125 was spotted and dried onto a nitrocellulose membrane in ten separate spots. After the spots dried, the membrane was placed in a conical 
tube with a solution containing approximately $2-3 \mathrm{nmol}$ of a random ssDNA library in binding buffer ( $100 \mathrm{mM} \mathrm{NaCl}, 20 \mathrm{mM}$ Tris pH 7.6, 2 mM MgCl2, $5 \mathrm{mM}$ $\mathrm{KCl}, 1 \mathrm{mM} \mathrm{CaCl} 2,0.02 \%$ Tween 20). Before exposure to the membrane, the library was heated at $90^{\circ} \mathrm{C}$ for ten minutes to separate any strands that were bound together then cooled on ice for five minutes.

Binding took place for two hours. The membrane was then thoroughly washed in binding buffer to remove unbound aptamer sequences. Squares from the membrane containing $\mathrm{CA}-125$ and bound aptamers were excised and placed in an elution buffer (40 mM Tris pH 8.0, $10 \mathrm{mM}$ EDTA, $3.5 \mathrm{M}$ urea, 0.02\% Tween 20) at $80^{\circ} \mathrm{C}$ for seven minutes with shaking. This was repeated twice with the aptamers being removed and pooled each time.

After pooling, the aptamers were purified using the QIAquick gel extraction kit from Qiagen (Valencia, CA). This purified, concentrated ssDNA was then used as the template in a PCR reaction that followed. The PCR reactions contained the following: $71.2 \mu \mathrm{L}$ of sterile DI water, $10 \mu \mathrm{L}$ of $10 \mathrm{mM}$ PCR reaction buffer from Invitrogen (Carlsbad, CA), $2 \mu \mathrm{L}$ of $10 \mathrm{mM}$ dNTPs, $5 \mu \mathrm{L}$ each of $100 \mu \mathrm{M}$ primers, $2 \mathrm{ng}$ of template, $3.8 \mu \mathrm{L}$ of $50 \mathrm{mM} \mathrm{MgCl} 2$, and $5 \mathrm{U}$ of Taq polymerase also from Invitrogen.

The PCR conditions were as follows: $94^{\circ} \mathrm{C}$ for three minutes for a hot start, followed by 30 cycles of $94^{\circ} \mathrm{C}$ for one minute, $51^{\circ} \mathrm{C}$ for one minute, and $72^{\circ} \mathrm{C}$ for one minute. An extension time of ten minutes at $72^{\circ} \mathrm{C}$ was carried out after the last cycle followed by cooling to $4^{\circ} \mathrm{C}$. 
It was necessary to determine the optimal conditions for the PCR cycle. The hot start temperature was determined by Invitrogen, the Taq polymerase manufacturer. An elongation temperature of $72^{\circ} \mathrm{C}$ is fairly standard and was never altered during experimentation. The annealing temperature was the temperature that needed to be experimentally determined. It is dependent on the primer composition and length and thus tends to be unique to each reaction. A range of temperatures in one degree increments ranging from $50^{\circ} \mathrm{C}$ to $60^{\circ} \mathrm{C}$ was used with $51^{\circ} \mathrm{C}$ creating the most efficient PCR reaction as judged by the relative brightness of the PCR products in a 4\% LMP agarose gel.

To make the PCR process as efficient and specific as possible the enzyme used to amplify the DNA was optimized. Along with the Taq polymerase from Invitrogen, HOT FIREPol ${ }^{\circledR}$ DNA polymerase (Solis Biodyne, Tartu, Estonia) and a high fidelity DNA polymerase from New England Biolabs (Ipswich, MA). The same quantity of all three enzymes, $5 \mathrm{U}$, was used in a PCR. To compare the enzymatic production, the PCR products were run through a denaturing polyacrylamide gel and the gel was then stained with ethidium bromide to visualize the products. The results, seen in Figure 30 indicated that the enzyme from Solis Biodyne produced a nonspecific band in the PCR product and that while the polymerase from Invitrogen and New England Biolabs both produced about the same amount of PCR product, the polymerase from Invitrogen would be more convenient to use due to it creating the mechanism required from molecular cloning during the amplification process, described in section 4.7 , 
whereas the New England Biolab enzyme did not and the PCR products would have to be modified before successful molecule cloning could occur.

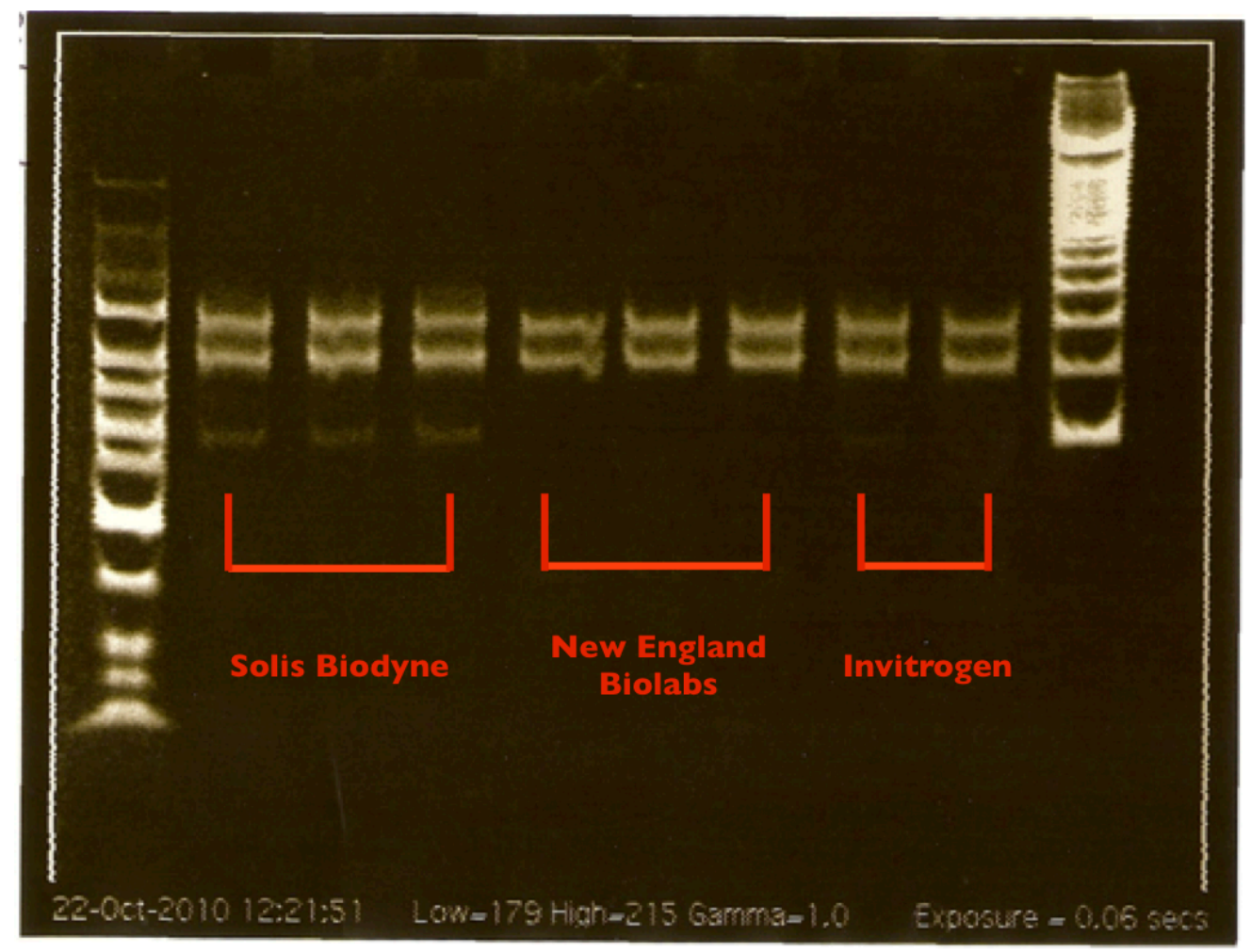

Fig 30. Results of PCR products created using different enzymes from different manufacturers visualized by ethidium bromide staining in a $10 \%$ polyacrylamide, $7 \mathrm{M}$ urea denaturing gel. The enzyme from Solis Biodyne creates a nonspecific product. The enzymes from New England Biolabs and Invitrogen created PCR products with the same efficiency, but the enzyme from Invitrogen was chosen because it created PCR products that did not require further modification before molecular cloning.

Following amplification the dsDNA products had to be separated so that the aptamers that bound to CA-125 could be pooled and used in the next selection round. A $10 \%$ polyacrylamide gel containing $7 \mathrm{M}$ urea was used to separate the PCR products. $10 \mu \mathrm{L}$ of PCR products were combined with $10 \mu \mathrm{L}$ of loading buffer ( $8 \mathrm{M}$ urea, $20 \mathrm{mM}$ Tris, $20 \mathrm{mM}$ EDTA) and heated at $95^{\circ} \mathrm{C}$ for five 
minutes to separate the products. This solution was then loaded into the gel and run at $132 \mathrm{mV}$ for 1 hour. The suggested voltage for gel electrophoresis is $8 \mathrm{~V} / \mathrm{cm}$ based on the length of the gel. The gel was then placed under a UV transilluminator where the binding band was easily visible due to the FITC label on one of the primers. These bands containing the binding sequences were excised from the gel.

The make-up of the denaturing polyacrylamide gel was experimented with. Clear separation of the DNA strands was key and that required the gel to run cleanly, leaving sharp bands that were truly separated. Gel combinations experimented with include a 10\% polyacrylamide gel containing $7 \mathrm{M}$ urea (Fisher Scientific, Fair Lawn, NJ) and 20\% formamide (Fisher Scientific), a 10\% polyacrylamide gel containing $10 \mathrm{M}$ urea, and a $10 \%$ polyacrylamide gel containing no denaturing reagent. The sample loading buffer was also experimented with.Variations included $10 \mathrm{M}$ urea, $10 \mathrm{M}$ urea with $20 \%$ formamide, and $8 \mathrm{M}$ urea with $20 \%$ formamide. Figure 31 represents the results of these variations.

It was determined that a denaturing polyacrylamide gel composed of $10 \%$ acrylamide/bis solution, 29:1 from Bio-Rad (Hercules, CA), 7 M urea, 1X TBE, and a 10:1 ratio of ammonium persulfate to TEMED (both purchased from BioRad) formed a gel that was effective in separating the double stranded PCR product. The best sample loading buffer was $8 \mathrm{M}$ urea, $20 \mathrm{mM}$ Tris (Invitrogen), and $20 \mathrm{mM}$ EDTA (Invitrogen). 
a)

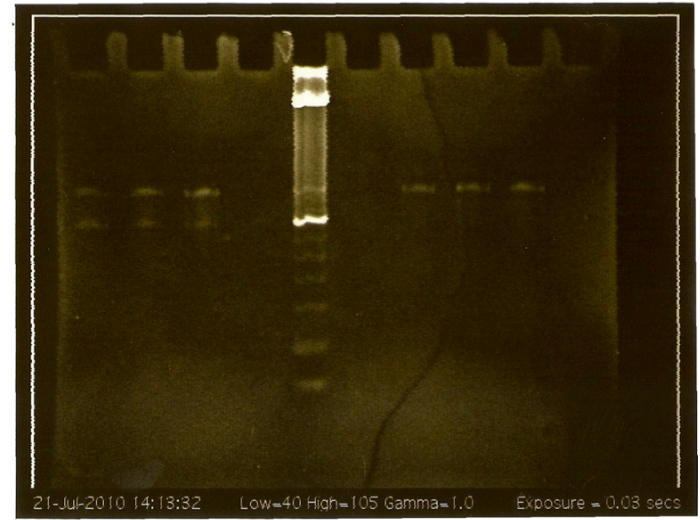

c)

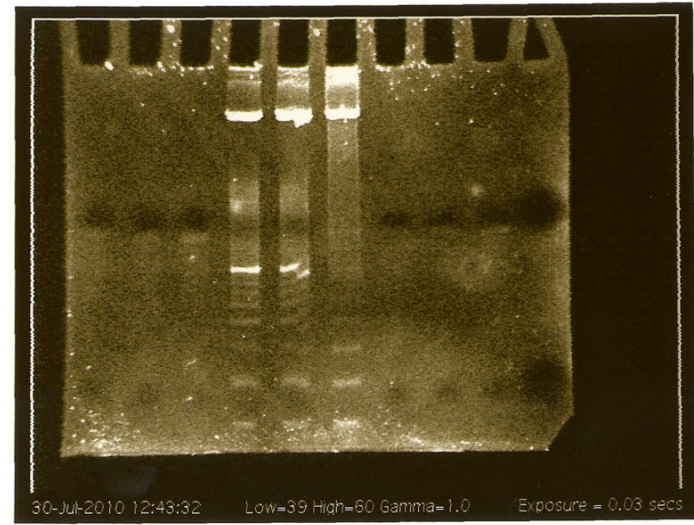

b)

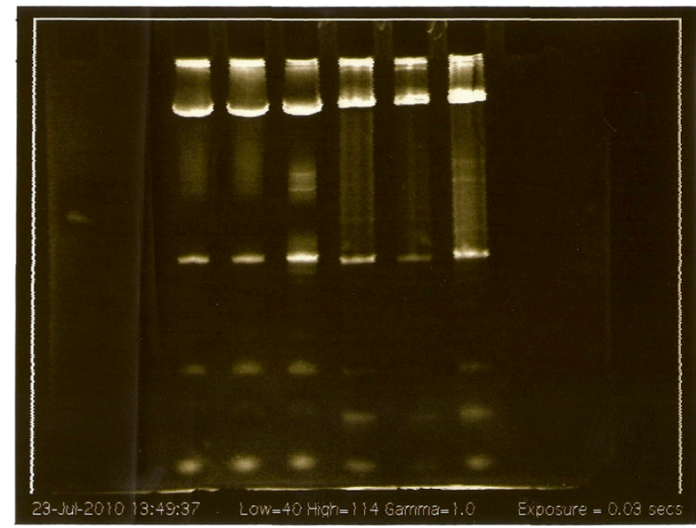

d)

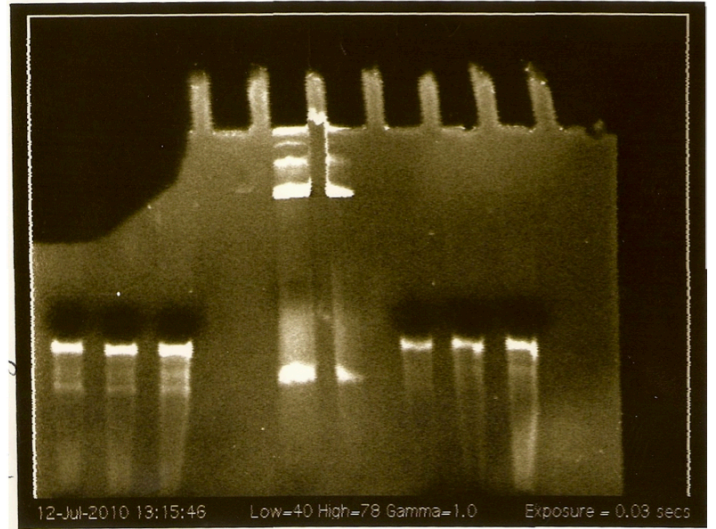

Fig 31. Gel composition determination. a, b) Denaturing polyacrylamide gel containing $10 \%$ polyacrylamide, $20 \%$ formamide, and $7 \mathrm{M}$ urea. Neither the a) PCR products nor the b) base pair ladders run smoothly. c) Denaturing polyacrylamide gel containing 10\% polyacrylamide and $10 \mathrm{M}$ urea. The high urea concentration created deposits of urea at the bottom of the wells preventing

the PCR products from entering the gel. d) Denaturing polyacrylamide gel containing $8 \%$ polyacrylamide and $7 \mathrm{M}$ urea. The low concentration of polyacrylamide fails to create enough pores for the PCR products to travel through. As a result, the smaller PCR products move through the gel more quickly than the dye and the larger PCR product is not visible. The larger products are located within the dark spots which is the dye.

To extract the DNA from the polyacrylamide gel the QIAEX II kit was purchased from Qiagen. The excised bands were placed in centrifuge tubes, crushed using a pipette tip, and then placed in $200 \mu \mathrm{L}$ of diffusion buffer $(300 \mathrm{mM}$ magnesium acetate $\mathrm{pH} 7.8,2 \mathrm{mM} \mathrm{NA}-\mathrm{EDTA}$ ) at $50^{\circ} \mathrm{C}$ for 30 minutes. 
After 30 minutes, the centrifuge tubes were spun down at 8000 RPM for one minute. The supernatant was collected and filtered using a $1 \mathrm{~mL}$ syringe and a $0.22 \mu \mathrm{m}$ filter. A volume of $6 \mathrm{X}$ the volume of filtered supernatant of Buffer QXI, which was provided with the kit, was added to the supernatant. An additional 10 $\mu \mathrm{L}$ volume of QIAEX II silicon beads were added. The solution was vortex mixed every two minutes for ten minutes to give the beads time to bind to the aptamers.

After incubation the tubes were centrifuged and the supernatant removed. The beads, now bound with the aptamers, were washed twice with $500 \mu \mathrm{L}$ of Buffer PE, also provided with the kit. The beads dried for ten minutes until the beads had just begun to turn white. $20 \mu \mathrm{L}$ of $10 \mathrm{mM}$ Tris, $\mathrm{pH}$ 8.0, was added to the beads and the pellet resuspended by vortex mixing. After five minutes the tubes were again centrifuged and the supernatant, containing the aptamers, was collected. This elution step was repeated twice to improve the yield.

These collected aptamers were then used in the next round of the SELEX procedure. Initially, in the second round, the entire pool of aptamers collected from the first SELEX round was used. After that, between 35-70 ng of collected aptamers were used in each subsequent SELEX round. Ten rounds of positive selection following this procedure were carried out.

4.6 Counter SELEX procedure. To create a more specific aptamer pool, six counter selection rounds followed the first ten rounds of positive selection. The counter selection rounds were carried out against bovine serum albumin (BSA), a 
common serum protein, and CEA. In all of these counter selection rounds the protein was spot dried onto the membrane just as the CA-125 had been in the positive selection rounds. However, for these rounds, any sequences bound to the membrane were removed from the selective aptamer pool. The remaining selective sequences were purified, used in a PCR, and separated using a denaturing polyacrylamide gel as outlined above.

4.7 Molecular cloning of aptamers. After the tenth round of positive selection and sixth round of counter selection, a PCR was used to create a pool of suitable aptamers for cloning. The PCR used unmodified versions of the primers used during the modified SELEX procedure. These primers did not contain FITC or poly A labels.

Using a TOPO TA Cloning Kit purchased from Invitrogen the aptamer pool was successfully cloned into E. coli bacteria. This particular kit was chosen based on its compatibility with the enzyme, Taq polymerase, used in the PCR process. Taq polymerase indiscriminately adds a single deoxyadenosine, represented by the letter A in DNA and RNA diagrams, to the 3' ends of PCR products as seen in Figure 32. The vector used with the TOPO cloning kit contains a single overhanging deoxythymidine, represented by the letter $\mathrm{T}$, on the 3 ' end. The binding between the deoxyadenosine and deoxythymidine enabled the aptamer to ligate with the vector and thus be inserted into the bacteria. Figure 33 contains a map of the $\mathrm{pCR}^{\circledR} 4-\mathrm{TOPO}^{\circledR}$ plasmid used in this kit. There are a 
total of 3956 nucleotides not including the PCR products being cloned. The PCR product is inserted into the plasmid at the $294^{\text {th }}$ nucleotide.

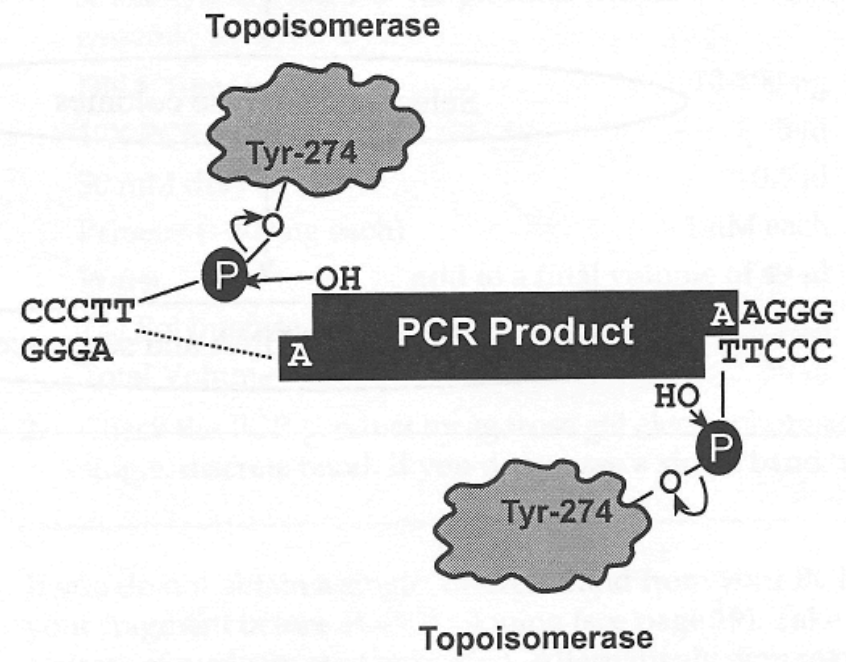

Fig 32. Cloning of PCR products. The topoisomerase adds a single adenosine nucleotide to the 3 ' end of the PCR products. This overhang is the mechanism for the PCR product's insertion into the plasmid DNA.
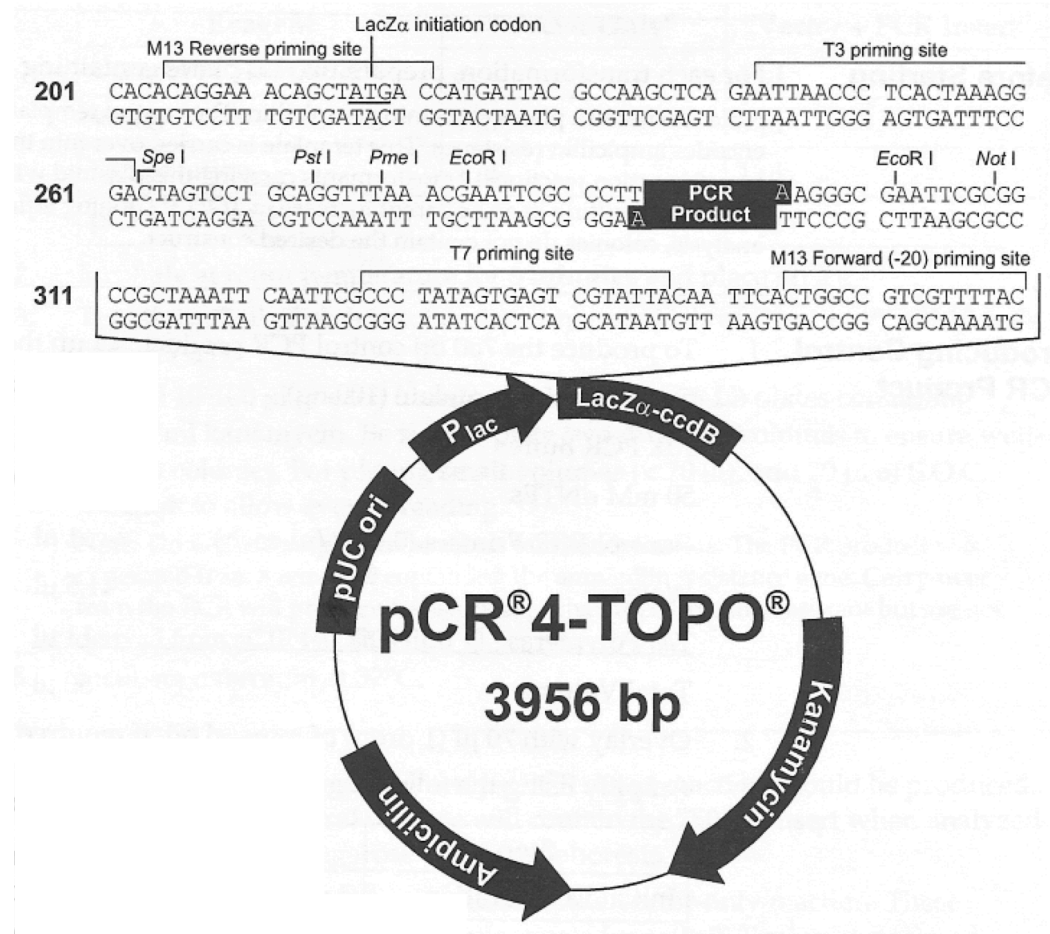

Fig 33. Map of the $\mathrm{pCR}^{\circledR} 4-\mathrm{TOPO}^{\circledR}$. The cloning site is located at bases 294-295. 
The cloning solution was then prepared by combining $4 \mu \mathrm{L}$ of the PCR products, $1 \mu \mathrm{L}$ of a salt solution that increased the number of transformants by 2-3 fold, and $1 \mu \mathrm{L}$ of the TOPO vector. This solution was gently mixed and incubated at room temperature for 30 minutes.

A vial of bacterial cells was thawed on ice and $2 \mu \mathrm{L}$ of the cloning solution was added to it and gently mixed. The cells and solution were then incubated on ice for 30 minutes. The cells were then heat shocked at $42^{\circ} \mathrm{C}$ for seconds and put on ice. $250 \mu \mathrm{L}$ of S.O.C. medium was added to the vial and it was placed in a $37^{\circ} \mathrm{C}$ incubator with horizontal shaking for one hour.

Agar plates prewarmed at $37^{\circ} \mathrm{C}$ for 30 minutes were spread with $25 \mu \mathrm{L}$ of the bacteria using sterilized glass beads. The plates were then incubated overnight at $37^{\circ} \mathrm{C}$. The following day 50 colonies were picked and cultured overnight in $3 \mathrm{~mL}$ of $\mathrm{LB}$ medium at $37^{\circ} \mathrm{C}$ to allow for further growth.

The plasmid DNA was extracted and purified using the Plasmid Miniprep Kit purchased from Invitrogen. The bacteria from the 50 colonies was pelleted and all medium removed. The cells were resuspended in $250 \mu \mathrm{L}$ of a buffer containing RNase A to remove the RNA from the samples. Lysis of the cells followed with the addition of $250 \mu \mathrm{L}$ of a lysing buffer. The cells were incubated in this buffer for five minutes at room temperature.

Immediate mixing with $350 \mu \mathrm{L}$ of a precipitation buffer took place. The samples were then spun down at 12000 RPM for ten minutes to separate the 
lysate from the lysis debris. The supernatant was extracted and loaded into a purification column. The column was placed on a vacuum manifold and the vacuum was applied. The column was washed with two different wash buffers containing ethanol. The column was placed inside a microcentrifuge tube and spun at 12000 RPM for one minute to remove any remaining wash buffer. The liquid recovered in the microcentrifuge tube was discarded and the column placed inside a new microcentrifuge tube.

To elute the DNA from the colony, $75 \mu \mathrm{L}$ of TE buffer (10 mM Tris, $1 \mathrm{mM}$ EDTA) preheated to $75^{\circ} \mathrm{C}$ was added to the center of the column and incubated at room temperature for one minute. The column was then centrifuged at 12000 RPM for two minutes. The liquid in the bottom of the centrifuge tube contained the purified plasmid DNA which was collected for all 50 colonies.

4.8 Return to the modified SELEX procedure. After analyzing the aptamer sequences contained in the 50 colonies, explained in further detail in chapter 5 , it was evident that the aptamer pool must be further refined before a suitable aptamer could be identified. Another 18 modified positive SELEX rounds were carried out. An altered version of the original binding buffer, called binding buffer II and containing $150 \mathrm{mM} \mathrm{NaCl}, 20 \mathrm{mM}$ Tris pH 7.6, $2 \mathrm{mM} \mathrm{MgCl}, 5 \mathrm{mM} \mathrm{KCl}, 1$ $\mathrm{mM} \mathrm{CaCl} 2,0.02 \%$ Tween 20 , was used to carry out nine rounds of positive selection. The idea behind altering the buffer by increasing the $\mathrm{NaCl}$ 
concentration from $100 \mathrm{mM}$ to $150 \mathrm{mM}$ was to increase the stringency of the binding conditions, resulting in a more specific aptamer pool.

After nine rounds using binding buffer II, the buffer was again altered by increasing the $\mathrm{NaCl}$ concentration, $150 \mathrm{mM}$ to $250 \mathrm{mM}$, and the Tween 20 percent, $0.02 \%$ to $0.05 \%$. Nine rounds of positive selection using this binding buffer, called binding buffer III, were carried out. The result of all of these modified SELEX rounds was a refined aptamer pool that was suitable for evaluation using molecular cloning techniques and sequence analysis.

4.9 Aptamer insert selection directly from molecular cloning. After the final 18 rounds of positive selection for CA-125 and the six counter selection rounds against BSA and CEA, the aptamer pool was reevaluated.

Using the pool of aptamers, a PCR was run using the unmodified versions of the primers. Molecular cloning using this PCR product was carried out as described in section 4.7 to the point of spreading the colonies using the glass beads and letting them grow overnight in a $37^{\circ} \mathrm{C}$ incubator. Before the cloning the size of the PCR products to be cloned was verified using a 4\% LMP agarose gel. Figure 34 estimates the size of most of the PCR products to be between 75 and $96 \mathrm{bp}$ in a 4\% LMP agarose gel. 


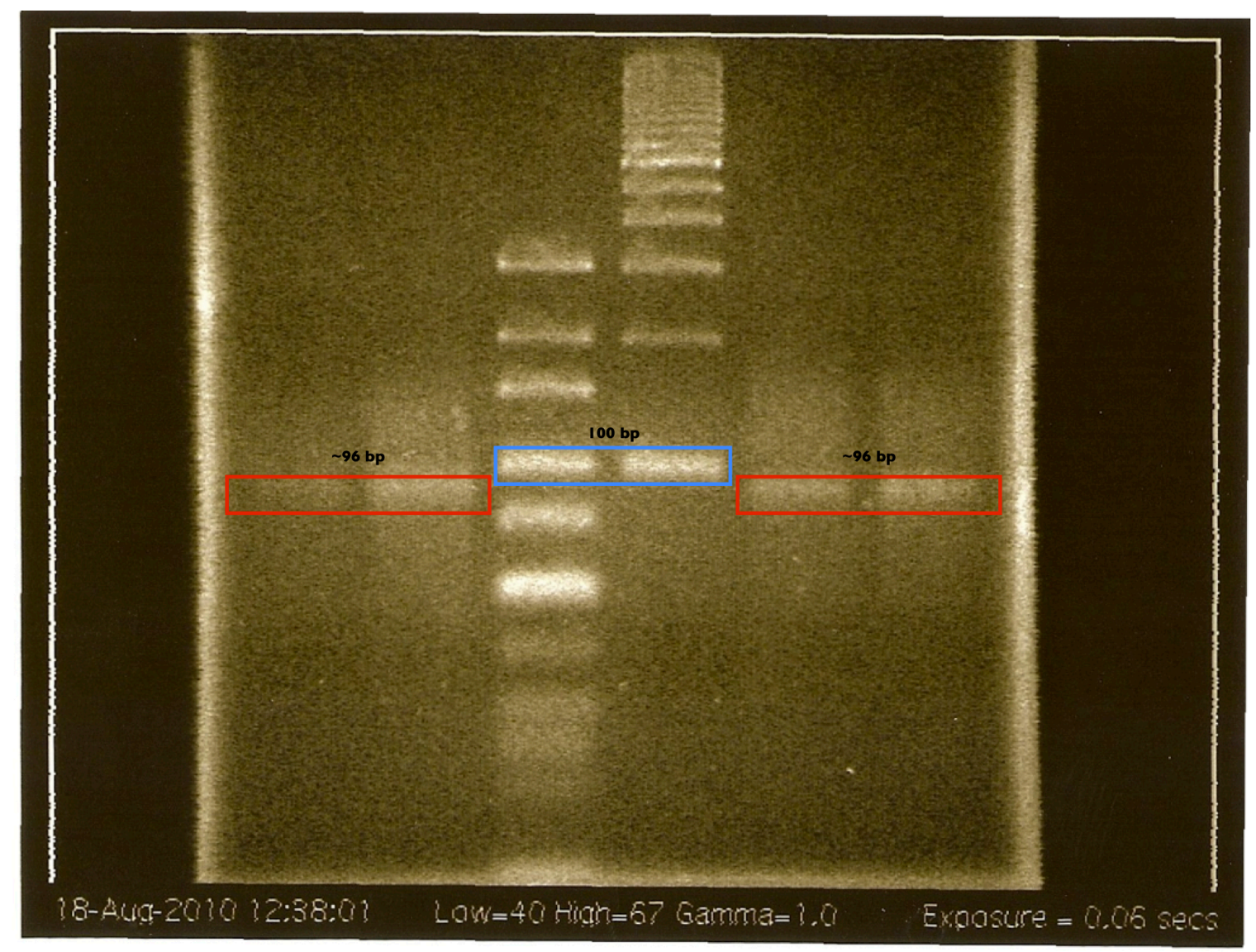

Fig 34. Verification of PCR insert size. A 4\% LMP agarose gel containing two DNA ladders and the PCR products used for molecular cloning. The gel is stained with ethidium bromide to visualize the contents. The blue box identifies the $100 \mathrm{bp}$ rung in the DNA ladders. The red box contains the PCR products whose length, $96 \mathrm{bp}$, falls just short of the $100 \mathrm{bp}$ mark. This served as an adequate benchmark to ascertain that the PCR products were appropriate for cloning.

The following morning 25 colonies were picked and placed in $25 \mu \mathrm{L}$ of sterile, DNase free water. At the same time that the colonies were picked an agar plate, pre-warmed to $37^{\circ} \mathrm{C}$, was gridded, labeled, and touched with a selected colony so that the colonies could be regrown and preserved for future use. The remaining unpicked colonies were sealed and placed at $4^{\circ} \mathrm{C}$ until further use. 
The 25 selected colonies were boiled for ten minutes. The cell debris was spun down at 13000 RPM for one minute and the supernatant, containing the plasmid DNA was collected.

A PCR was run using the plasmid DNA as the template and using the modified primers to amplify the plasmid insert. Because the primers bind to the priming site of the aptamer insert, only the aptamer was copied during this PCR. The PCR conditions outlined in the SELEX Procedure, section 4.5, chapter proved to be optimal for the plasmid PCR and they were followed exactly. Just as in the SELEX procedure, section 4.5, the PCR products were run through a $10 \%$ denaturing polyacrylamide gel and the binding strands, identifiable by the FITC label, were excised from the gel and purified using the Qiagen Qiaex II kit.

A total of 50 colonies were chosen to have their aptamer insert extracted and collected following this procedure. By doing this it was possible to analyze the affinity and specificity for CA-125 that each aptamer exhibited and to determine the best aptamers to have sequenced and incorporated for use in a biosensor. 


\section{Chapter 5:}

Aptamer screening through sequence analysis and ELISA development 
5.1 Analysis of cloned sequences. The purified plasmid DNA was sequenced by the MMI DNA Analysis Core Facility located at Oregon Health \& Science University. The returned sequences went through a screening process to determine the accuracy and quality of the sequencing data, whether there were any repeated sequences that were cloned, what the most common DNA motifs present in the sequences were, and what similarities existed in the secondary structure of the cloned sequences.

The sequencing data was verified using 4Peaks from Mekentosj. This software loads the raw sequence data and by creating peaks for every recognized nucleotide allows visual inspection of the quality and accuracy of the sequence that is identified. If the peaks are narrow, pronounced, and do not overlap, then the nucleotide that has been identified at that particular location in the sequence is correct. Figure 35 compares data representing poor and accurate sequence identification as analyzed by 4peaks. If the peaks are broad with small amplitudes and overlap with neighboring peaks, there may exist a misidentification of the nucleotide at that position in the sequence. 
a)

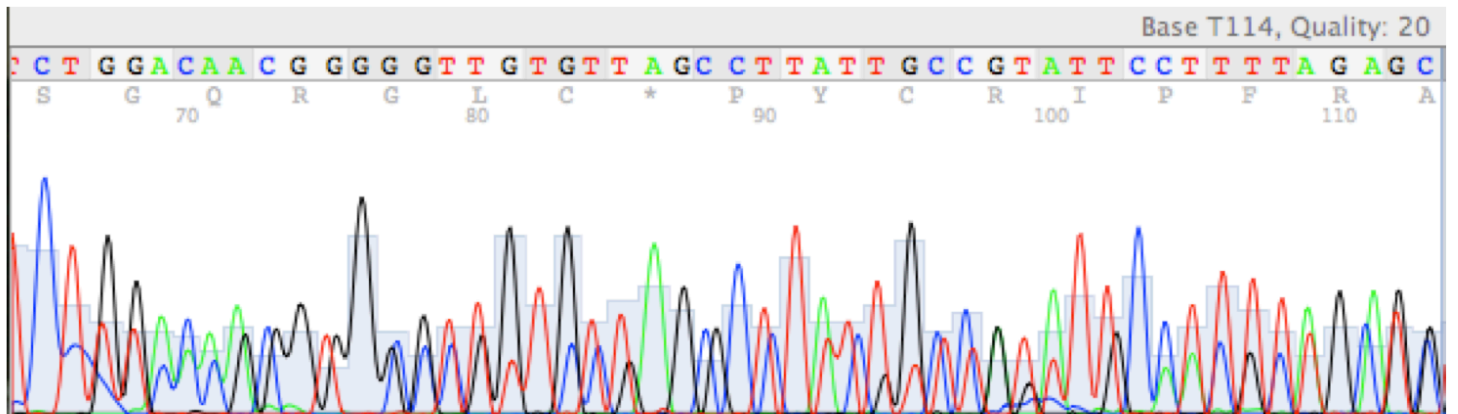

b)

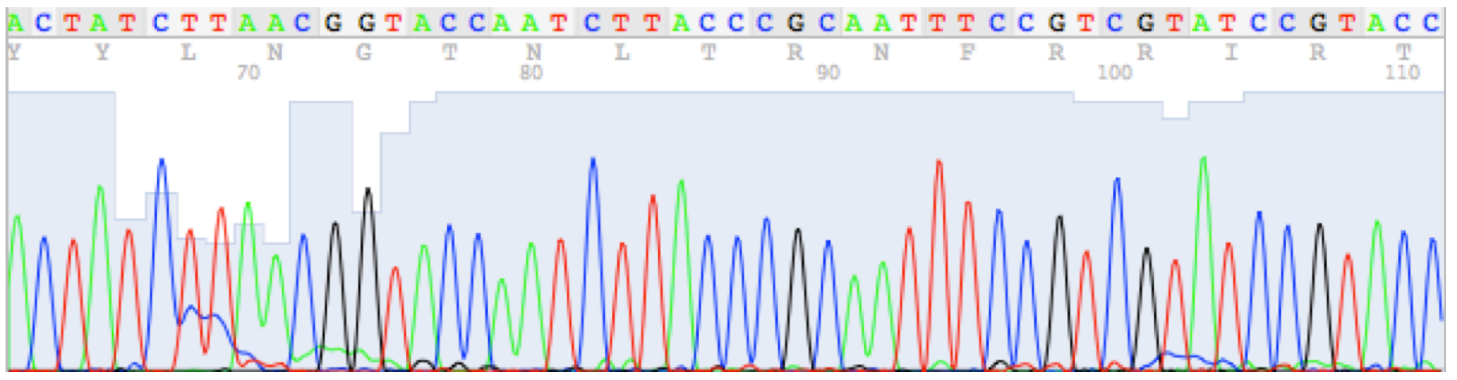

Fig 35. Analysis of sequencing data using 4peaks software. It is important to analyze the quality and accuracy of the sequencing data for aptamer analysis. Without proper identification of the aptamer sequence it is impossible to further analyze the structure of the aptamer. a) Poorly identified sequencing data. Broad, overlapping peaks signal possible misidentification of the nucleotide at that position in the sequence. b) Accurate identification of the sequence. Individual peaks for each nucleotide are present at each position in the sequence with minimum noise.

The 50 sequences initially chosen from the first cloning attempt were examined using 4Peaks with 47 of those containing highly accurate sequence data. The 4Peaks software also enables the user to search out a particular string of nucleotides. The cloned sequences, $96 \mathrm{bp}$, were able to be identified from the entirety of the plasmid DNA, which was 3,956 bp, based on the primer sequences. Both the individual binding aptamers and the reversed complimentary strands to the binding aptamers were discovered within the plasmid DNA by searching for the primer sequences. The sequences containing the primer whose modified version contained the FITC label were the binding sequences. The sequences containing the primer whose modified version 
contained the poly A label was the reverse compliment sequence of the binding sequence. These sequences were identified, taken the reverse compliment of if necessary, and translated into a FastA format.

Once in this format, the sequences were analyzed using ClustalX, a DNA alignment program from University College Dublin. The sequences were compared to each other using the program and several alignments of the sequences were attempted without any success. It was found that two of the sequences were duplicates of each other.

Lacking an overall alignment, motifs were sought using DiLiMoT. DiLiMoT is a web based server that searches files for short repeating sequences called linear motifs [54]. These motifs range from 3-8 nucleotides long. Common motifs indicate a commonality in the sequence structure that could be responsible for the binding between the sequence and the target. After analyzing the 47 sequences with DiLiMoT, sequences containing the one or more of the ten most common motifs were collected for further analysis. From these sequences the ones that contained three or more of these ten most common motifs were collected for structural analysis. The number of sequences that remained for more analysis at this point was nine.

The structure of the aptamer is responsible for its ability to bind to a target protein so it was to be expected that there would be similarities in the secondary structure of the aptamers that contained the most common motifs. mfold is a web based server that determines the possible secondary structures of DNA 
sequences by computing the minimum free energy [55]. The number of secondary structures ranged between 1 and 7 possible structures for each sequence. Each of these possible structures was compared to all other possible structures for the nine remaining sequences. Figure 36 contains examples of the structures of the selected aptamers derived from mfold.

The possible structures were made up of loop and stem structures. Each possible structure was first compared to the other possible structures for the same sequences to find any conserved regions that were constant in every structure. These conserved structures were compared to the other possible structures for the other sequences. 

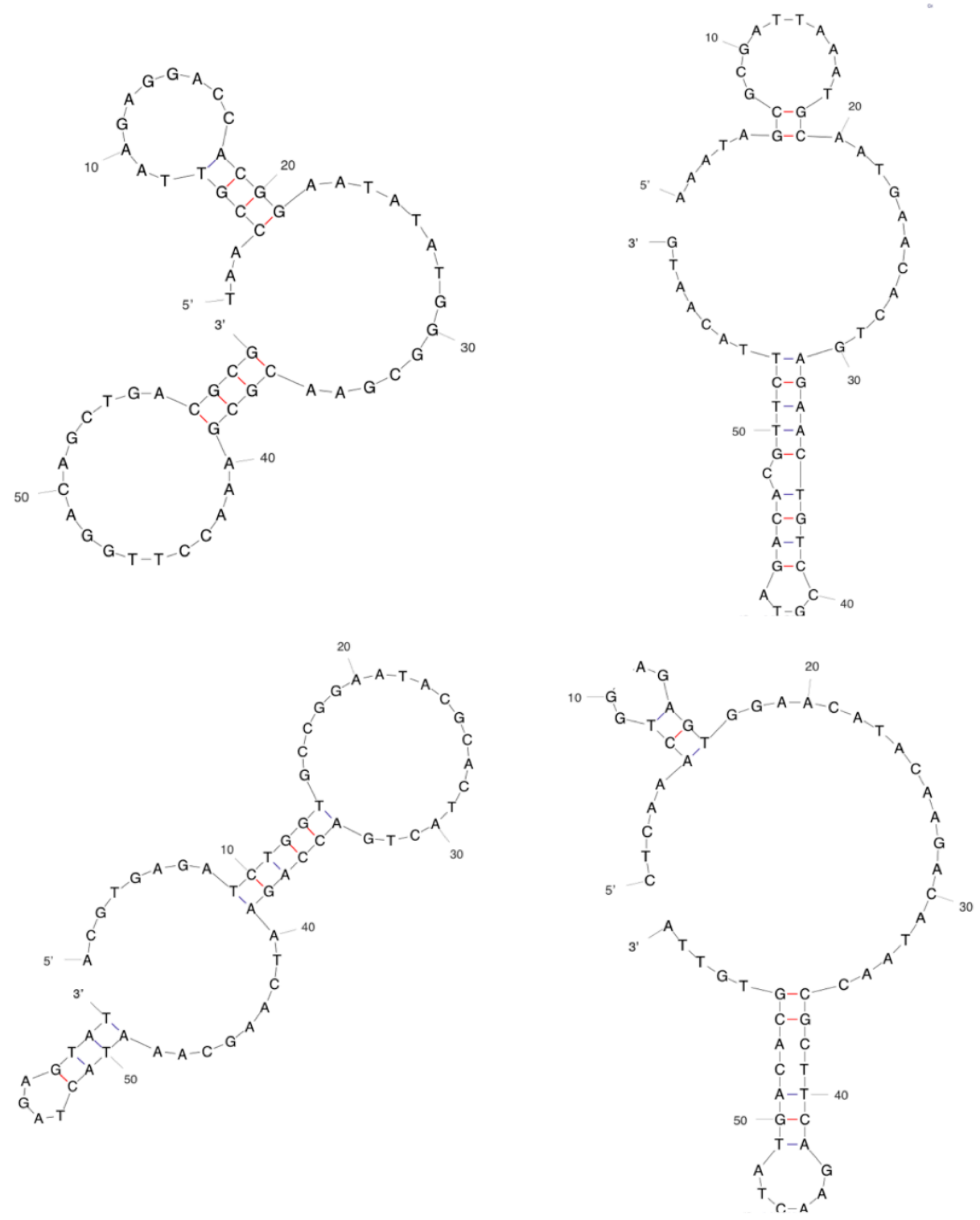

Fig 36. Examples of the secondary structures of the aptamers obtained from mfold. These are four possible structures for four different aptamers. Secondary structures like these were investigated for any similarities that might be present among the sequences to find the mechanism responsible for binding between the aptamers and CA-125.

The five sequences that contained the most structural similarities with the other sequences were selected for synthesis. The synthesis was done by IDT. The entire $96 \mathrm{bp}$ aptamer, including the primer regions, was synthesized, HPLC 
purified, and biotinylated. Further analysis through ELISA, described in section 5.4, was used to determine the aptamer most suitable to binding with CA-125.

5.2 ELISA background. The enzyme-linked immunosorbent assay, ELISA, is a commonly used assay to detect a target in a liquid sample. Generally, a binding reagent, such as an antigen, is coated onto the bottom of the wells of a high binding polystyrene 96-well plate. A liquid sample, possibly containing the ligand for the binding reagent, such as an antibody, is incubated in the wells and given ample time to bind to the binding reagent.

The binding complex is then built up by the addition of other ligands to the well with one of the ligands containing an enzyme capable of inducing a color change when combined with a particular substrate. The color change is directly correlated to the amount of material bound in the well.

Washing steps, usually with a nonreactive buffer containing a small percentage of a mild detergent, occur after every ligand additive step to remove any unbound ligating reagents. Nonspecific sites of the well without the initial binding reagent bound to them must also be blocked. The wells are high binding and it is possible for a ligand not specific to the original binding reagent to bind to the well and create a false signal. ELISAs have been developed to detect a large number of targets including antigens, antibodies, drugs, allergens, toxins, and hormones. 
5.3 ELISA procedure for aptamer pool analysis. As the aptamer pool was developed and refined it was necessary to evaluate the progression of the pool's selectivity and affinity for the target protein, CA-125. ELISAs were developed for this express purpose. Figure 37 is a composite drawing showing the components of the ELISA developed for testing the aptamer pools.

The bottom of a high binding 96-well plate was coated with $50 \mu \mathrm{L}$ of $2 \mu \mathrm{g} /$ $\mathrm{mL}$ solution of CA-125 in carbonate/bicarbonate buffer and incubated onto the plate overnight at room temperature. Several washing steps in PBST and PBS removed any unbound CA-125. The nonspecific binding sites were blocked with a $5 \%$ solution of skim milk in the binding buffer that was used to bind the aptamers to the CA-125. A volume of $350 \mu \mathrm{L}$ of the blocking solution was placed in each well of the plate so that the well was filled and all sites effectively blocked. This blocking solution was incubated in the plate for two hours at room temperature. Washing with PBST and PBS followed to remove the blocking solution.

The aptamers, containing an FITC label and ranging in concentration from 10nM to $0.3125 \mathrm{nM}$ in binding buffer depending on the exact ELISA being carried out, were placed in the bottom well at a volume of $50 \mu \mathrm{L}$. Binding between the aptamers and CA-125 took place overnight at room temperature. Before being placed in the well, the aptamers were heated at $95^{\circ} \mathrm{C}$ for five minutes and immediately placed on ice to ensure the aptamers were separated. The plate was washed with PBST and PBS to remove any unbound aptamers. 
The well was next filled with $50 \mu \mathrm{L}$ of a 1:1000 solution of an antiFITC mouse antibody in $0.1 \%$ skim milk in binding buffer. The antiFITC was purchased from Sigma Aldrich and the 1:1000 solution was half of the manufacturers suggested dilution as it was unclear if the suggested amount of $5 \mu \mathrm{g} / \mathrm{mL}$ of FITC was present in the well. This solution was incubated in the well for two hours at room temperature. The plate was washed with PBST and PBS to remove the excess antibody.

The final component of this sandwich ELISA was the addition of $50 \mu \mathrm{L}$ of 1:5000 monoclonal anti-mouse IgG conjugated with horseradish peroxidase (HRP). This antibody was purchased from Millipore (Temecula, CA) and prepared at the manufacturer's suggested dilution. Again, this secondary antibody solution was made in $0.1 \%$ skim milk in binding buffer. It incubated in the well plate for one hour at room temperature. 


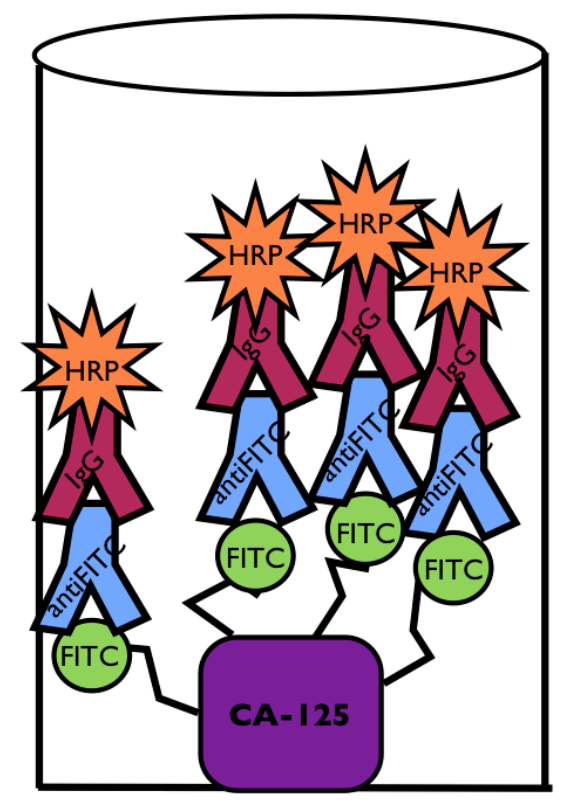

Fig 37. Diagram of the ELISA for testing the aptamer pools. CA-125 is coated on the bottom of the well. FITC-labeled aptamers, represented by angular black lines, bind to the CA-125. An antiFITC antibody binds to the FITC and a protein IgG conjugated with HRP binds to the antiFITC. A TMB substrate is added to the wells, reacts with the HRP, and produces a readable signal. Image is not to scale.

Excessive washing with PBST and PBS followed this incubation as it was imperative to remove any unbound HRP, thus minimizing the potential of a false signal. The wells were then filled with $50 \mu \mathrm{L}$ of TMB substrate solution, purchased from Sigma Aldrich, which induced a color change over a period of 30 minutes at room temperature. Finally, $50 \mu \mathrm{L}$ of the stop reagent, purchased in conjunction with the TMB substrate, was added quickly to the wells to stop the enzymatic reaction responsible for the color change. The absorbance of each well was measured at a wavelength of $450 \mathrm{~nm}$ using an ELISA plate reader (BioTek, EL 800). The absorbance values were blank adjusted and analyzed using Open Office Spreadsheet. 
5.4 ELISA procedure for synthesized aptamers. Once the five selected aptamers had been synthesized, HPLC purified, and biotinylated, and ELISA was developed to determine which aptamer exhibited the strongest affinity for CA-125. Figure 38 is a diagram of the ELISA developed for this purpose.

The five chosen aptamers had been selected from the aptamer pool that had undergone ten positive selection rounds for CA-125, three counter selection rounds against bovine serum albumin (BSA), and three counter selection rounds against CEA. To determine the effectiveness of the positive and counter selection rounds, the wells of the ELISA plate were coated with $50 \mu \mathrm{L}$ of $2 \mu \mathrm{g} / \mathrm{mL}$ CA-125, CEA, and BSA in carbonate/bicarbonate buffer and incubated overnight at room temperature. Washing with PBST and PBS followed. The nonspecific binding sites were blocked using $350 \mu \mathrm{L}$ of $5 \%$ skim milk in binding buffer for two hours at room temperature. The plate was washed with PBST and PBS.

Each of the five biotinylated aptamers were made at concentrations of 50 , $10,2,0.4,0.08$, and $0.016 \mu \mathrm{g} / \mathrm{mL}$, or a $1 / 5$ series of dilutions, in binding buffer. A volume of $50 \mu \mathrm{L}$ of all six aptamer concentrations was placed in the wells with CA-125 absorbed onto them. The three highest aptamer concentrations were placed in wells with CEA absorbed onto them and the highest concentration of each sequence was placed in the wells containing BSA. The wells containing CEA and BSA also received $50 \mu \mathrm{L}$ of each aptamer concentration. 
After the aptamers were incubated overnight at room temperature the wells were washed with PBST and PBS and $50 \mu \mathrm{L}$ of a $10 \mu \mathrm{g} / \mathrm{mL}$ streptavidinHRP conjugate in $0.1 \%$ of skim milk in binding buffer was added to the wells and incubated over the course of an hour at room temperature. The wells were washed with PBST and PBS and $50 \mu \mathrm{L}$ of TMB substrate was added to the wells. After 30 minutes $50 \mu \mathrm{L}$ of the stop reagent was added to each well and the absorbance of each well on the plate was read at $450 \mathrm{~nm}$ by the ELISA plate reader. The resulting ELISA showed no binding between the synthesized aptamers and CA-125 or CEA. The ELISA was repeated three times and still no binding occurred. At this point it was determined that the aptamer pool would need to be further refined before a suitable aptamer could be determined.

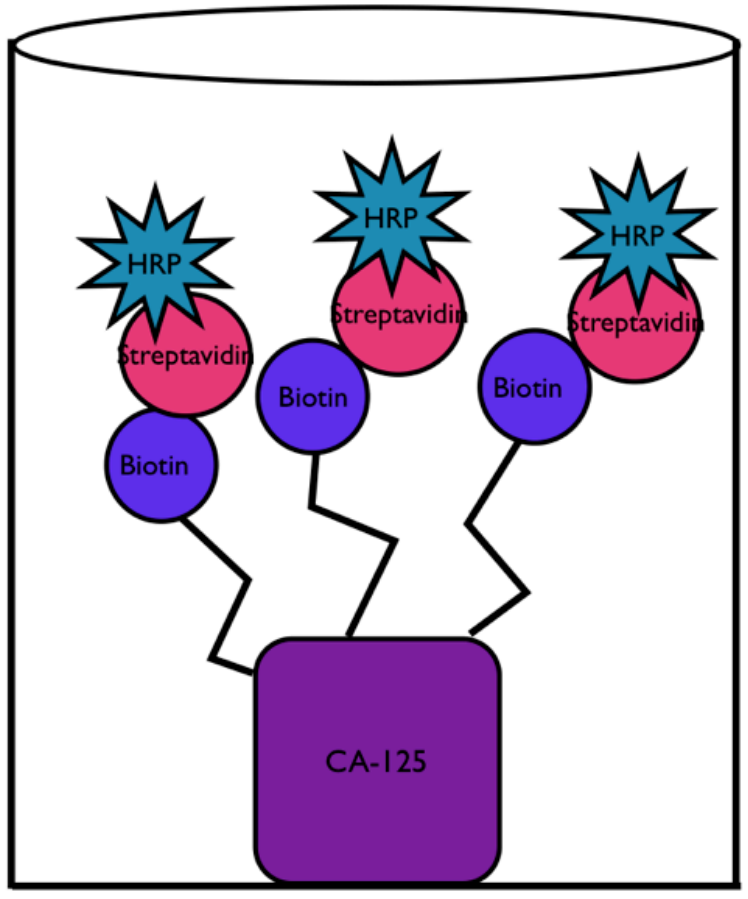


Fig 38. Overview of ELISA for synthesized aptamers. CA-125 is attached to the bottom of the well. The synthesized aptamers, represented by angular black lines, contain a biotin label and bind to the CA-125. A streptavidin-HRP conjugate is added to the wells and binds to the biotin.

Finally a TMB substrate that interacts with the HRP is added to the well producing a color change. Image is not to scale.

5.5 ELISA procedure for plasmid DNA inserts. After the aptamer pool was refined through an additional 18 rounds of positive selection and molecular cloning had been carried out using this refined pool, the aptamers extracted from 50 colonies were analyzed using ELISA. The purpose of this was to determine which of the individual aptamers showed binding to CA-125 and of those which ones showed a higher specificity for CA-125 than for CEA before sequencing was done. Because all of these aptamers were FITC labeled, the ELISA procedure was identical to the ELISA procedure for aptamer pools described in section 5.3. All 50 colonies showed binding with CA-125 and 14 of those showed considerably more binding with CA-125 than CEA. The results can be reviewed in section 6.5. These 14 colonies were sequenced and the results analyzed as discussed in section 5.1 . 


\section{Chapter 6:}

Results and discussion 


\subsection{Results of biosensor for CA-125 detection. A label-free biosensor was}

developed for fast, sensitive detection of CA-125. A sensing layer that immobilized monoclonal CA-125 antibodies for capturing CA-125 was developed on the IDEs. Varying concentrations of CA-125 were detected by the sensor and the resulting change in impedance was measured.

The change in impedance was derived from the change in impedance values when nothing is bound to the sensing layer and when CA-125 has been bound to the sensing layer. The results are presented in Figure 39. Impedance values were obtained over a range of frequencies between $50 \mathrm{~Hz}$ and $1 \mathrm{MHz}$ and compared. The most sensitive signal coupled with the least amount of standard deviation was obtained at $100 \mathrm{~Hz}$.

An increase in impedance values was observed to correspond with increasing concentrations of CA-125. The sensor was able to detect CA-125 down to a concentration of $10 \mathrm{U} / \mathrm{mL}$ and up to a concentration of $80 \mathrm{U} / \mathrm{mL}$. Distinguishable signals were generated by the sensor when each concentration was detected. The sensor is capable of determining whether the amount of CA-125 present is within the diseased range. The clinical benchmark for ovarian cancer diagnosis when evaluating CA-125 level in serum samples is $35 \mathrm{U} / \mathrm{mL}$ and above. The signal generated by the sensor at $20 \mathrm{U} / \mathrm{mL}$, which is below the threshold, is differentiable from the signal generated by the sensor at $40 \mathrm{U} / \mathrm{mL}$ which is just above the diagnosis concentration. 
More testing is needed to be done beyond this narrow range to determine the dynamic range of the sensor. Judging by the large change in impedance observed for $10 \mathrm{U} / \mathrm{mL}$ it appears that the sensor could have the capacity to detect lower concentrations. The signal generated by $80 \mathrm{U} / \mathrm{mL}$ of $\mathrm{CA}-125$ indicates that detection of greater concentrations may be possible. The signal is not saturated between 40 and $80 \mathrm{U} / \mathrm{mL}$ implying that differentiable signals may be obtained beyond $80 \mathrm{U} / \mathrm{mL}$. However, the percent change in impedance is considerably lower between 40 and $80 \mathrm{U} / \mathrm{mL}$ than between 20 and $40 \mathrm{U} / \mathrm{mL}$ so it is feasible that the sensor is already working towards the top end of its dynamic range.

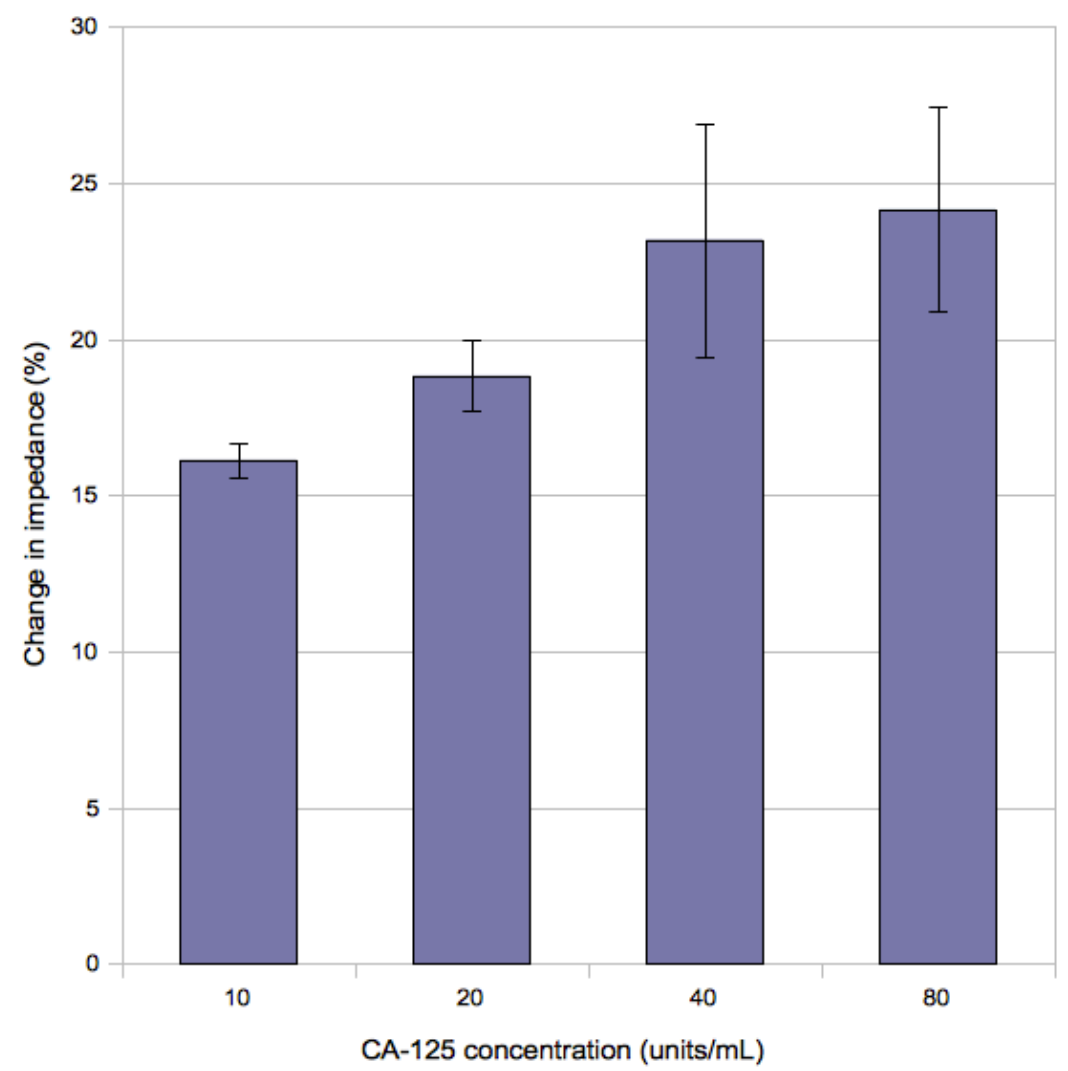

Fig 39. Detection of CA-125 in spiked buffer. There is a measurable increase in the change in impedance with the increasing concentration of CA-125. The biosensor produces a differentiable signal between 20 and 40units $/ \mathrm{mL}$ making it effective in differentiating between diseased and non-diseased levels of CA-125 within the clinical range for diagnosis. All data collected at $100 \mathrm{~Hz}$. 
This sensor was also used to detect CA-125 in 5\% rabbit serum. The serum was spiked with CA-125 at various concentrations and the data was collected and analyzed in the manner described in the beginning of this section. The results are in Figure 40. In a complex sample the sensor does not appear capable of detecting CA-125 below a concentration of $40 \mathrm{U} / \mathrm{mL}$ like it does when the sample is spiked buffer. A strong signal is obtained at $40 \mathrm{U} / \mathrm{mL}$ which is used for disease diagnosis. There is little deviation in the signals indicating that the sensor is capable of reproducing a reliable signal even in complex samples. More data would need to be collected on this sensor before the reliability could be properly established. Far fewer of these sensors were tested than the ones for spiked buffer samples, which had larger signal deviations.

A comprehensive review of this data gave the impression that while this sensor was a promising step towards being able to diagnose disease in clinical samples, it would be necessary to detect CA-125 below $40 \mathrm{U} / \mathrm{mL}$ so that clear signals would be generated inside and outside of the diseased concentration range. It was this impetus that would result in the development of an aptamer to replace the monoclonal CA-125 antibody in the sensing layer. 


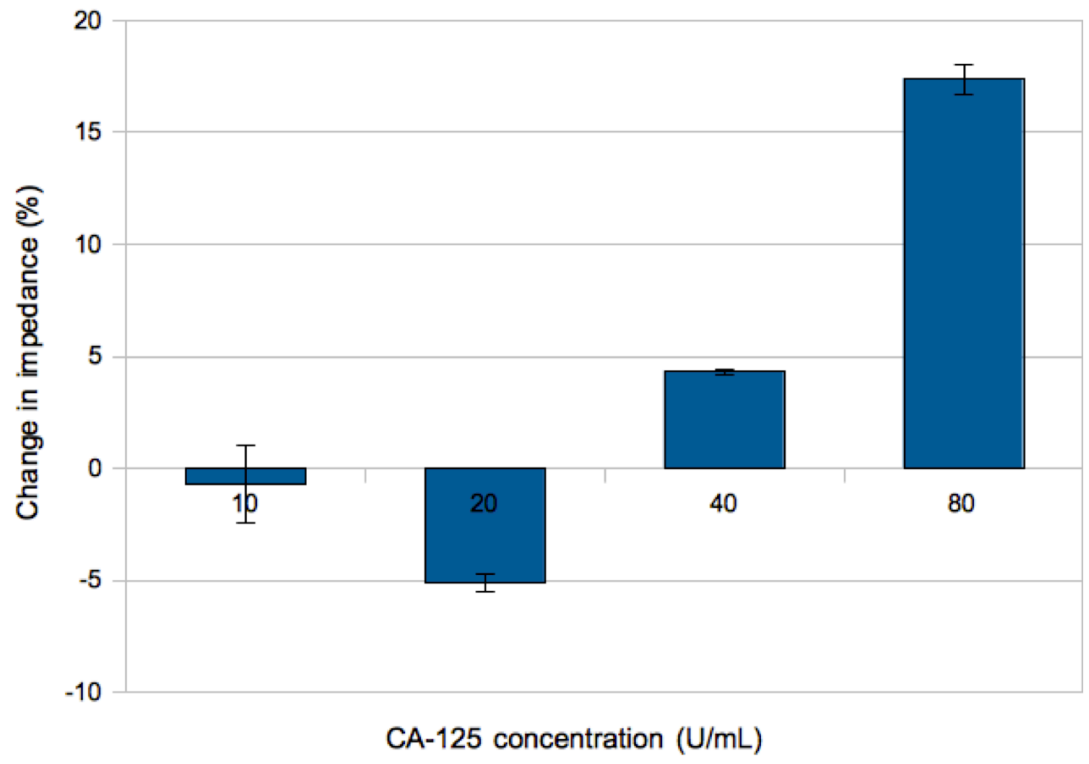

Fig 40. CA-125 detection in 5\% rabbit serum. 5\% rabbit serum was spiked with varying concentrations of CA-125. The sensor is unable to detect CA-125 below $40 \mathrm{U} / \mathrm{mL}$. There is little deviation in the signals generated and the sensor is capable of generating a signal within the diseased range. All data collected at $500 \mathrm{~Hz}$.

As CA-125 is such an important biomarker for ovarian cancer there has been considerable work put into the development of a biosensor for its detection. One of the first efforts was a biosensor that used cyclic voltammetry, another electrochemical method, to monitor the changes in current over a small voltage range when CA-125 was detected by the sensor [56]. This sensor employed a sandwich immunoassay to detect CA-125. This sandwich assay included magnetic nanoparticles conjugated with an HRP-antiCA-125 complex as the sensing probes and thionine-HRP doped silica nanoparticles as recognition elements. These particles contained both the enzyme and electron mediator labels making the addition of labels into the sensor very convenient. Detection of CA-125 concentrations in spiked buffer were between 0.1 and $450 \mathrm{U} / \mathrm{mL}$. 
The advantage of this sensor is its large dynamic detection range. However, the use of nanoparticles and enzymatic labels increase the cost of the device. They also increase the detection time. Two 18-minute incubation steps are required before a signal can be generated whereas the label-free sensor described above requires only ten minutes for signal generation.

A sensor very similar to EIS sensor described in this section was recently reported. Like the EIS sensor, a SAM was developed on gold electrodes and a monoclonal antibody to CA-125 was used to detect concentrations of CA-125 in spiked buffer, spiked serum, and whole blood [57]. Cyclic voltammetry and differential pulse voltammetry, both electrochemical detection methods, were used to obtain the data. The reported detection range was 0.1 to $100 \mathrm{U} / \mathrm{mL}$ in increasing factors of ten in spiked buffer and spiked serum and from 0.1 to $10 \mathrm{U} /$ $\mathrm{mL}$ in whole blood.

This sensor has several advantages over the EIS sensor described at the beginning of this section. It is capable of detecting a higher and lower concentration range and it is capable of detecting CA-125 in whole blood. However, the detection time for this sensor is $\mathbf{4 0}$ minutes, four times longer than the ten minutes required by the EIS sensor. There is also no detection reported within the key diagnostic range. The signal change reported between 10 and 100 $\mathrm{U} / \mathrm{mL}$ is substantial, but there is no signal change reported for concentrations that lie between those concentrations. Any sensor used in a clinical setting would be required to differentiate within that concentration gap. 
The nonfaradaic EIS biosensor described in this section is one of a number of promising new biosensors being developed to detect $\mathrm{CA}-125$ that could eventually lead to earlier detection of ovarian cancer.

\subsection{Results of biosensor for CEA detection. A label-free biosensor was} developed for the rapid, sensitive detection of CEA in spiked buffer. Nonfaradaic EIS data was collected on all eight IDEs for CEA concentrations ranging from 10 $\mathrm{pg} / \mathrm{mL}$ to $10 \mu \mathrm{g} / \mathrm{mL}$. The sensing layer was created using monoclonal CEA antibodies.

Impedance measurements were made before CEA was exposed to the sensing layer and after CEA had been incubated on the electrodes. The change in impedance, which is what is used to quantify the signal generated by the sensor in Figure 41, is derived from the change in impedance values when nothing is bound to the sensing layer and when CEA has been bound to the sensing layer.

There is an increase in the change in impedance that is linearly correlated with the increasing concentration of CEA. The signal appears to saturate at 10 $\mathrm{ng} / \mathrm{mL}$ as the change in impedance fails to increase for CEA concentrations beyond that. It is assumed that at $10 \mathrm{ng} / \mathrm{mL}$ of CEA all available binding sites on the sensing layer have been occupied and there is nowhere for the excess CEA to bind. As such, $10 \mathrm{ng} / \mathrm{mL}$ is considered the top end of the dynamic detection range for this sensor. 
It would appear that it could be possible for the sensor to detect concentrations of CEA lower than $10 \mathrm{pg} / \mathrm{mL}$, but more testing would have to be done to ascertain that information. If $10 \mathrm{pg} / \mathrm{mL}$ is the lower end of the dynamic detection it would still make the sensor capable of very sensitive detection as 10 $\mathrm{pg} / \mathrm{mL}$ is three order of magnitude below the concentration used for diagnosis.

The sensor is effective at detecting CEA within the clinical range of diagnosis which is at $10 \mathrm{ng} / \mathrm{mL}$ and above. There is a substantial change in the percentage change in impedance between 1 and $10 \mathrm{ng} / \mathrm{mL}$.

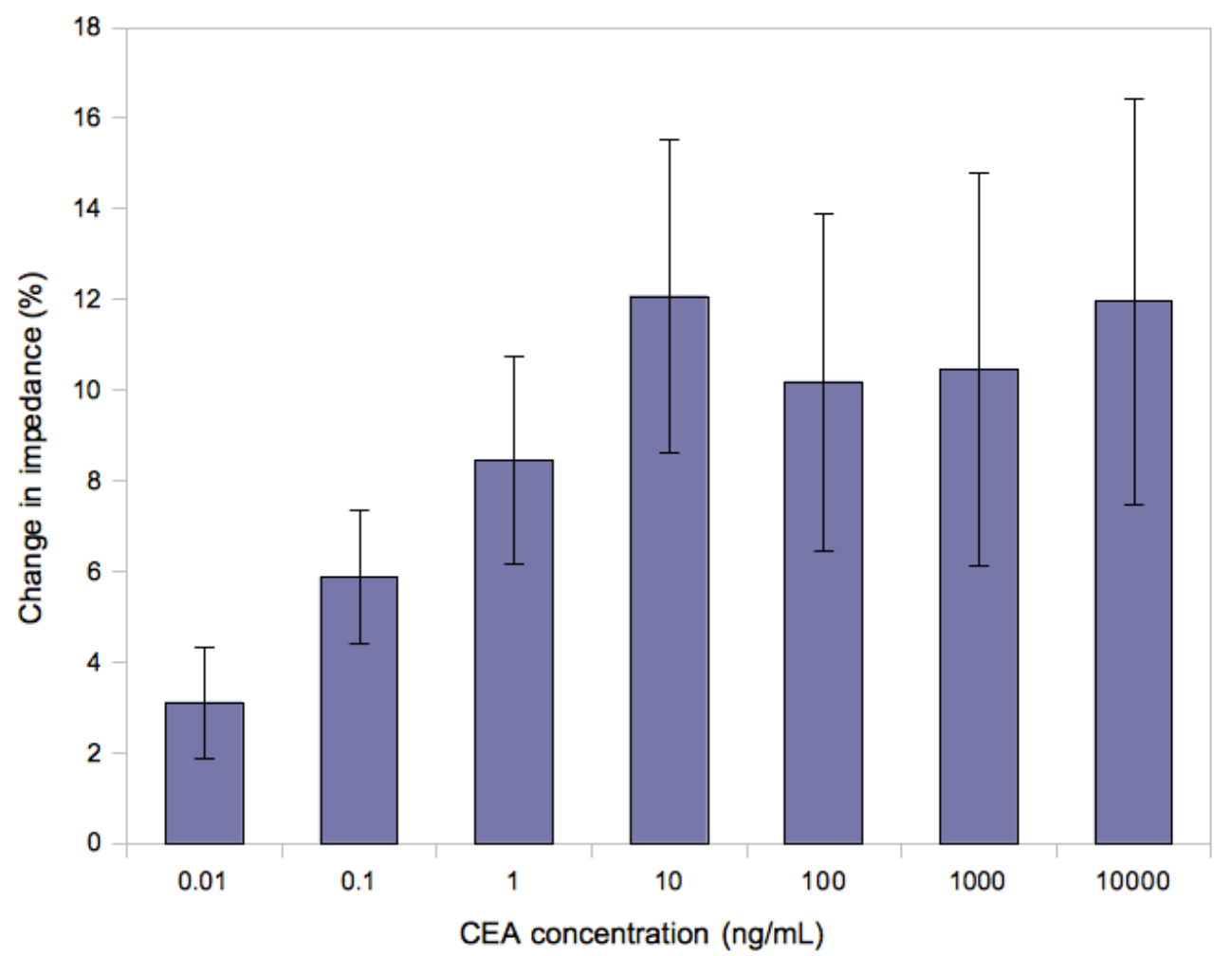

Fig 41. Detection of CEA in spiked buffer. There is a direct linear relationship between the increase in change in impedance and the increase in the amount of target CEA detected by the biosensor. The signal levels out at a concentration of $10 \mathrm{ng} / \mathrm{mL}$ after which presumably the binding sites available on the sensing layer have been saturated. The biosensor if able to discriminate between 1 and $10 \mathrm{ng} / \mathrm{mL}$, making it effective in differentiating between diseased and non-diseased levels of CEA within the clinical range for diagnosis. All data collected at $100 \mathrm{~Hz}$. 
The sensor described above was also used to detect CEA concentrations in $5 \%$ rabbit serum. The data was collected and analyzed according to the procedure described in this section. The results are in Figure 42. It is difficult to determine the sensor's capabilities of detecting CEA in a complex sample if there are any at all. There is not an increase in the percentage change in impedance for every CEA concentration and it is inconsistent in detecting CEA at both low and high concentrations. Not as many sensors were developed to detect CEA in $5 \%$ rabbit serum as there were to detect CEA in spiked buffer and it may be that the IDEs were defective in some way. More testing would be needed to determine if these signals were a consequence of the fabrication process or the sensor's inability to detect CEA in a complex sample.

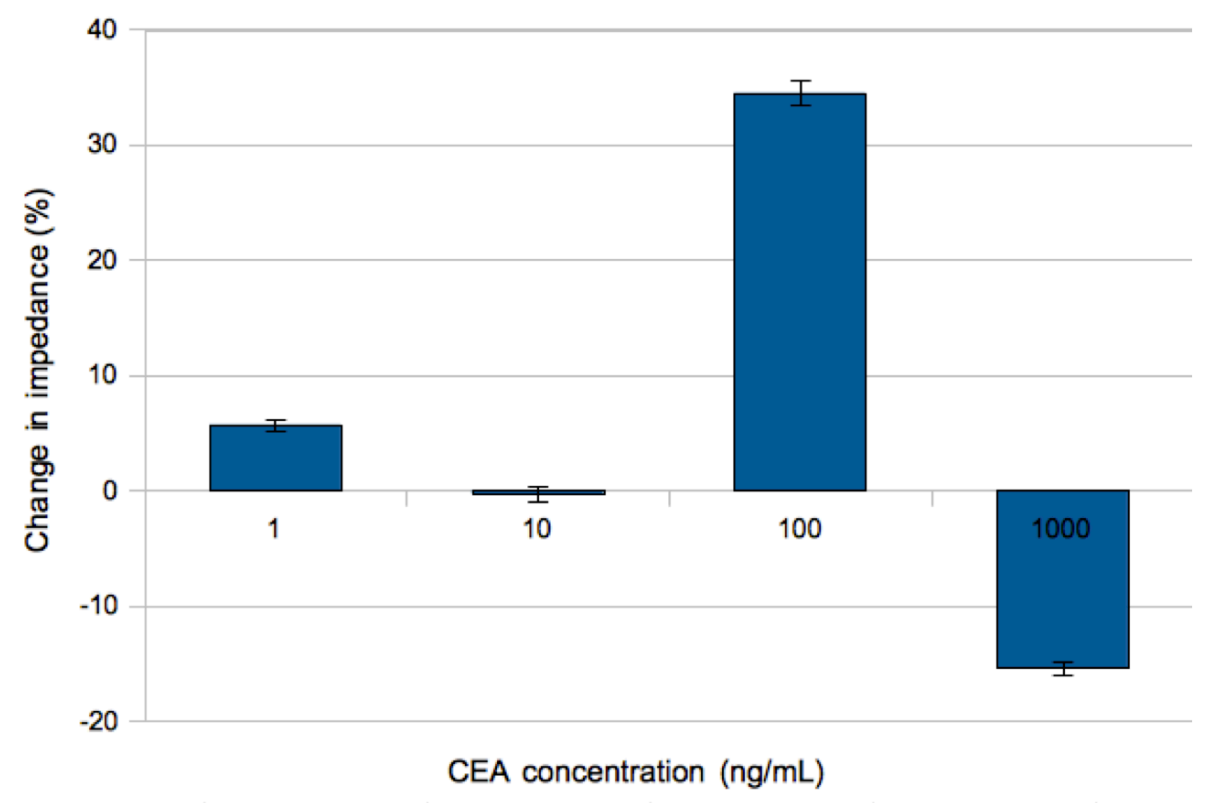

Fig 42. CEA detection in 5\% rabbit serum. 5\% rabbit serum was spiked with CEA at various concentrations and detected by the sensor. The results are inconclusive. There is not consistent detection at low concentrations or high concentrations. This may be a result of the sensor's inability to detect CEA in a complex sample or the fabrication process. 
CEA has recently gained traction as a biomarker for several types of cancer and work is being done to incorporate it into a biosensor platform. However, there has not been much development in way of developing an impedimetric sensor to detect CEA individually making it difficult to compare detection achieved with this sensor to detection reported by other groups. As it tends to be indicated in a number of diseases it is usually used in a sensor designed to detect a large number of targets.

Recently there was reported an optical biosensor for detecting CEA using surface plasmon resonance. A CEA antibody was immobilized onto gold electrodes using a SAM [58]. Known concentrations of CEA in spiked buffer solution were incubated on the electrodes for the period of one hour and the responding signals were measured. With this sensor, the dynamic range of CEA detection was reported to be between 3 and $400 \mathrm{ng} / \mathrm{mL}$.

Though the SPR sensor has many advantages, such as it's high end concentration for dynamic detection, it also has many disadvantages when compared to the CEA sensor described above. The SPR sensor does not detect CEA below a concentration of $3 \mathrm{ng} / \mathrm{mL}$ whereas the EIS sensor detects CEA down to $10 \mathrm{pg} / \mathrm{mL}$. The time required for the SPR signal is one hour compared to ten minutes for the EIS sensor. The development of this nonfaradaic EIS sensor is promising step in the ability to detect CEA in patients with ovarian cancer while the disease is still in the early stages. 
6.3 Results of biosensor for He4 detection. There were two labeled faradaic EIS biosensors developed to detect He4. The initial sensor used a secondary antibody to amplify the signal and the second sensor used an enzymatic reaction to amplify the signal. The first sensor incorporated a monoclonal He4 antibody into the sensing layer on the electrode. Varying concentrations of He4, already combined with a secondary $\mathrm{He} 4$ antibody held at a constant concentration of 10 $\mu \mathrm{g} / \mathrm{mL}$ to form an immunocomplex, was exposed to the sensor. The Nyquist plots were obtained for every concentration and $R_{\mathrm{ct}}$ values obtained from them using Z-view. These $R_{c T}$ values were then compared to the $R_{C T}$ values obtained from the electrodes after the sensing layer was developed on them but before any binding with the He4 immunocomplex. These $\mathrm{R}_{\mathrm{cT}}$ values were compared and a resulting measurement of the change in $R_{C T}, \Delta R_{C T}$, was made.

These $\Delta R_{\mathrm{CT}}$ values were compared for every concentration of $\mathrm{He} 4$ as seen in Figure 43 . At concentrations below $50 \mathrm{ng} / \mathrm{mL} \mathrm{He} 4$ is not detected by the sensor. He4 concentrations below that resulted in the $R_{C T}$ values dropping. This may have been due to the removal of some of the sensing layer during the washing procedures. Even when the $\mathrm{R}_{\mathrm{ct}}$ values did increase, such as for $\mathrm{He}$ concentrations of 50 and $100 \mathrm{ng} / \mathrm{mL}$, the increase was smaller than the decrease in $R_{C T}$ values at lower concentrations. Even for concentrations 50 and $100 \mathrm{ng} /$ $\mathrm{mL}$ the standard error was so high that it could not be stated that there was any detection of $\mathrm{He} 4$ by the biosensor. 


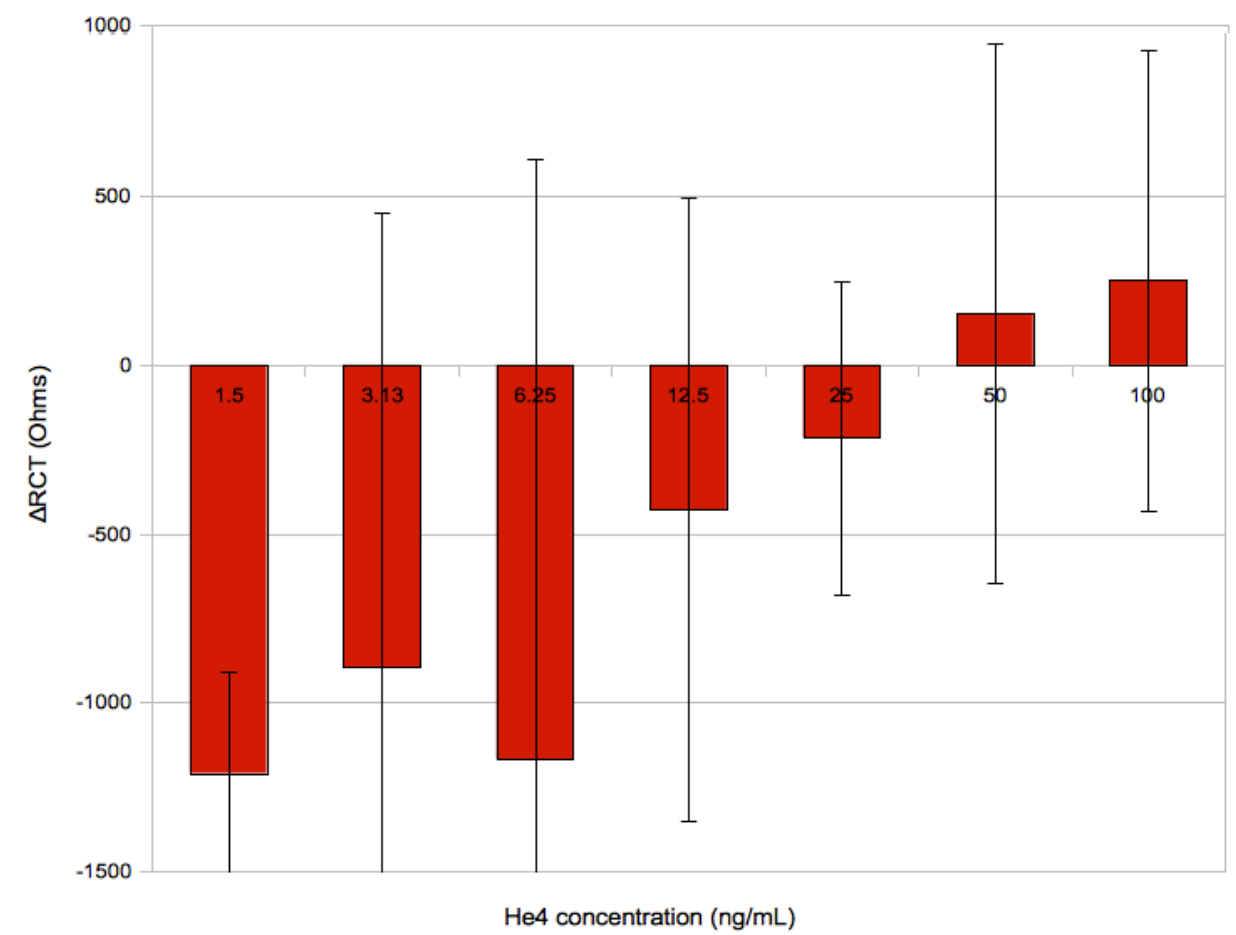

Fig 43. Detection of He4 using faradaic EIS. An immunocomplex consisting of a varying $\mathrm{He} 4$ concentration bound to a secondary monoclonal antiHe4 antibody, held at a constant concentration of $10 \mu \mathrm{g} / \mathrm{mL}$, is detected by the sensor. An $R_{\mathrm{Cr}}$ value is obtained from each Nyquist plot and compared to the $R_{\text {Cт }}$ value obtained from the sensor before it was exposed to the immuncomplex. The resulting change in $R_{c T}, \Delta R_{c T}$, is compared for each immunocomplex concentration. The sensor failed to detect the He4 immunocomplex with any consistency or reliable change in signal.

After having no success with the first He4 sensor, a second sensor was developed. This sensor relied on the formation of several layers of materials on the sensing layer and used faradaic EIS to obtain impedance measurements. A sensing layer containing the same monoclonal antibodies as used in the first $\mathrm{He} 4$ biosensor was developed on the electrodes. Varying concentrations of $\mathrm{He} 4$, already combined with a biotinylated secondary $\mathrm{He} 4$ antibody held at a constant concentration of $10 \mu \mathrm{g} / \mathrm{mL}$ to form an immunocomplex, was exposed to the sensor. Next a streptavidin-HRP conjugate at a concentration of $1 \mu \mathrm{g} / \mathrm{mL}$ was exposed to the sensor. Finally, a TMB substrate was exposed to the sensor. 
Faradaic EIS data was collected after the incubation of each reagent and Nyquist plots were created. $R_{\mathrm{ct}}$ values were extrapolated from the Nyquist plots and the $\Delta R_{C T}$ values obtained. Figure 44 compares these $\Delta R_{C T}$ values for the development of every layer on the electrodes. The blue data is the $\Delta R_{c t}$ values when the biotinylated immunocomplex has been detected by the sensing layer. The red data are the $\Delta R_{\mathrm{CT}}$ when the streptavidin-HRP has bound to the biotinylated antibody already captured by the sensing layer. Finally, the green data are the $\Delta R_{C T}$ values when the TMB substrate had reacted with the HRP already on the sensing layer.

The $\Delta \mathrm{R}_{\mathrm{CT}}$ values for the immunocomplex increase linearly with the increase in $\mathrm{He} 4$ concentration until a concentration of $25 \mathrm{ng} / \mathrm{mL}$. After this, the signal seems to have saturated as there may be no more available binding sites with the monoclonal antibodies. As a result, the $\Delta R_{C T}$ values fall off. The $\Delta R_{C T}$ values are relatively low for $\mathrm{He} 4$ concentrations below $12.5 \mathrm{ng} / \mathrm{mL}$, but they increase rapidly between 12.5 and $25 \mathrm{ng} / \mathrm{mL}$. The $\Delta R_{C T}$ values increase for every He4 concentration when the streptavidin-HRP and TMB labels are added. This is particularly true for $1.56 \mathrm{ng} / \mathrm{mL}$, the lowest concentration of He4. At some He4 concentrations, such as $6.25 \mathrm{ng} / \mathrm{ML}$ and $25 \mathrm{ng} / \mathrm{ML}$ the addition of the layers did not greatly amplify the $\Delta R_{C T}$ values. This is to be expected for the He4 concentration of $25 \mathrm{ng} / \mathrm{mL}$ as the signal generated by the immunocomplex alone seems to have saturated at that point. 
The clinical range for ovarian cancer diagnosis using He4 is still being determined. Estimates range from $150 \mathrm{pM}$ to $500 \mathrm{pM}$ which equates to between 3 and $10 \mathrm{ng} / \mathrm{mL}$. The sensor is able to detect He4 within this range. There are clearly differentiable $\Delta \mathrm{R}_{\mathrm{CT}}$ values for $3.125 \mathrm{ng} / \mathrm{mL}$ and $12.5 \mathrm{ng} / \mathrm{mL}$. Even without the additional streptavidin-HRP and TMB layers, detection of the biotinylated immunocomplex generates differentiable signals within this range. These labeling layers do greatly amplify the signal at $12.5 \mathrm{ng} / \mathrm{mL}$ which is above any cutoff level currently under investigation.

The use of labels is not ideal for any biosensor. They increase the cost of the device and the time required for the sensor to generate a signal. They can also modify the binding ability of the protein they are attached to which in this sensor a secondary antibody, though any effect that the biotin label may have had on the secondary antibody's ability to bind to $\mathrm{He} 4$ is insignificant enough that the antibody can still bind to $\mathrm{He} 4$ and the $\mathrm{He} 4$ can then be detected by the sensing layer. Using the formation of biolayers to enhance the signal presents an additional problem. The creation of these layers depends on previous binding steps, i.e. He4 must bind to the sensing layer for the streptavidin to bind to the biotin and the enzymatic reaction between the TMB substrate and HRP is dependent upon the streptavidin-biotin binding. However, the formation of these layers does enable detection of He4 down to a concentration of $1.56 \mathrm{ng} / \mathrm{mL} /$ 


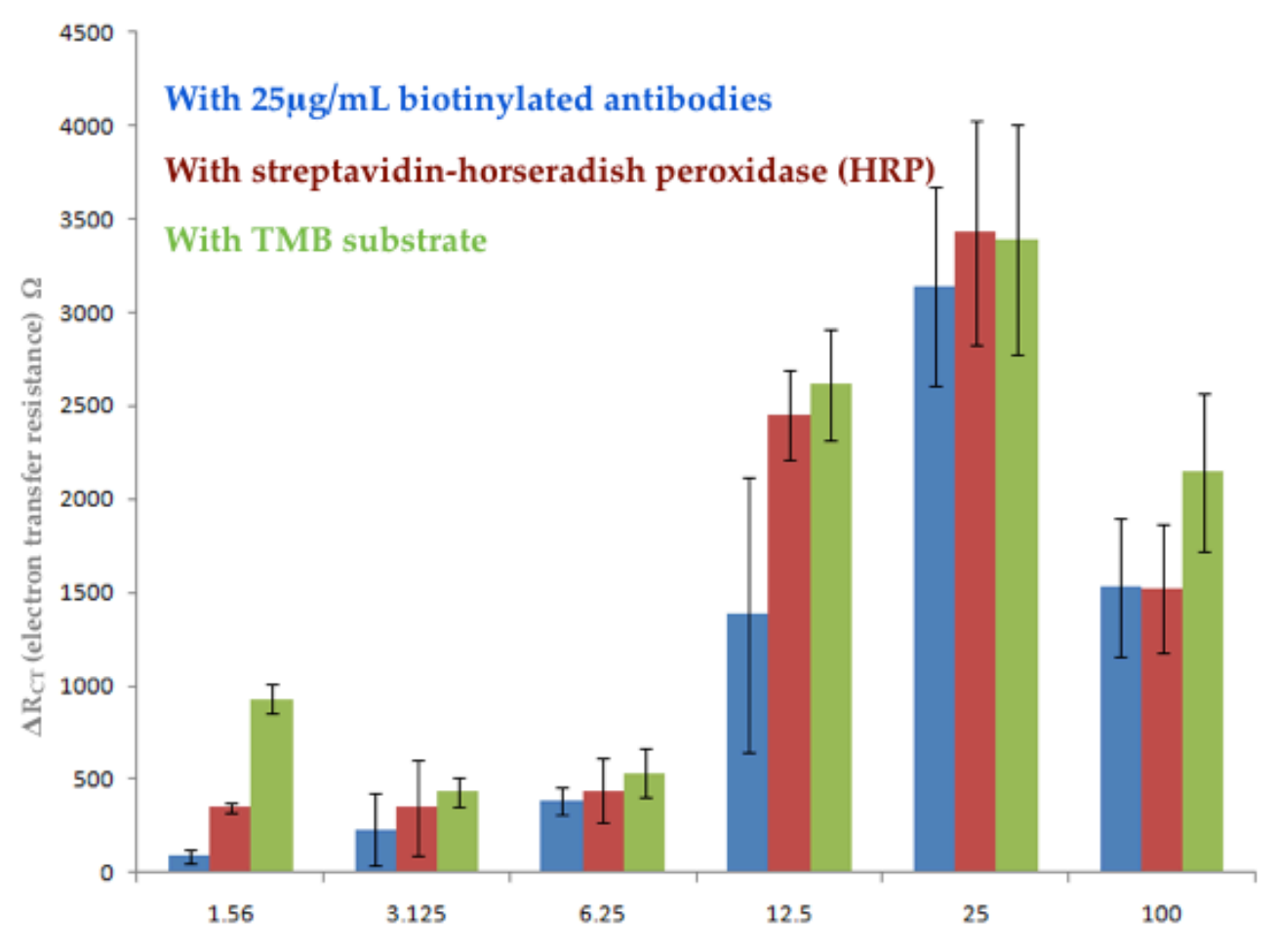

He4 concentration (ng/mL)

Fig 44. Labeled detection of $\mathrm{He} 4$ in spiked buffer using biotinylated antibodies. Changes in the electron transfer resistance, $\Delta R_{C T}$, were extrapolated from Nyquist plots using Zview and compared for the formation of every layer on the sensor. Varying concentrations of He4 which had already been allowed to bind to a fixed concentration of $10 \mu \mathrm{g} / \mathrm{mL}$ of a secondary biotinylated antibody were exposed to the sensing layer on the chip. Next, a layer of streptavidin-HRP conjugate at a concentration of $1 \mu \mathrm{g} / \mathrm{mL}$ was bound to the sensing layer, altering the $\Delta R_{C T}$ values. Finally, TMB substrate was exposed to the biosensor. During its interaction with HRP, the TMB became an insoluble precipitate, further altering the $\Delta R_{C T}$ values.

There is very little work currently being reported in the development of a biosensor for the detection of $\mathrm{He} 4$ making it difficult to ascertain how effective this sensor is when compared to other sensors. The nearest thing that can be found is a talk given at the Trends in Nanotechnology conference in 2008 by Darby Kozak [59]. In it, optically encoded nanoparticles are used to detect He4 and mesothelin in a high-throughput biosensor platform. Beads coupled with single-chain variable fragments specific to $\mathrm{He} 4$ and mesothelin are used to 
capture those target proteins. A fluorescent antibody is then used to generate an optical signal.

Using this system, He4 concentrations between 75 and $1100 \mathrm{ng} / \mathrm{mL}$ were detected in spiked buffer, $20 \%$ serum, and whole serum. This data appears to never have been published. The range of detection far surpasses the concentrations used to determine the presence of disease making it ineffective in a clinical situation. The other detraction is that it, like the biosensors described in this section, employs labels to generate a signal. Again, this leads to an increase in cost and time of detection and possible loss in binding capabilities of the labeled molecule.

\subsection{Results of multiplexed biosensor for CA-125 and CEA detection. A}

multiplexed sensor was developed to detect CA-125 and CEA simultaneously using nonfaradaic EIS. There are a total of eight IDEs located on the biosensor chip and they are conveniently separated into two groups of four IDEs. Four of the electrodes were functionalized using a monoclonal CA-125 antibody in the sensing layer to detect CA-125 and the remaining four electrodes were functionalized using a monoclonal CEA antibody to detect CEA.

As varying concentrations of CA-125 were exposed to the sensor, EIS data was collected in the manner described in 3.3. Data was collected on electrodes functionalized to detect $\mathrm{CA}-125$ and on the other electrodes that were functionalized to detect CEA. By collecting data in this manner it was possible to 
quantify the nonspecific binding that could occur with a multiplexed sensor that was designed to detect two ovarian cancer biomarkers at once while injecting only one sample into the device.

Figure 45 is the multiplexed biosensor's detection of CA-125 in spiked buffer. The change in impedance was measured at $500 \mathrm{~Hz}$ and is derived from the change in impedance values when nothing is bound to the sensing layer and when CA-125 has been bound to the sensing layer. The data in purple is the change in impedance measured from the electrodes specified for the detection of CA-125 and the data in orange is the change in impedance measured on the electrodes specified for the detection of CEA. It can be seen that the change in impedance increases linearly with the increase in CA-125 concentration for both the specific and nonspecific electrodes. As with the sensor for detection of CA-125 as described in 6.1, the multiplexed sensor generated distinct signals in the diseased and nondiseased clinical ranges. Between $20 \mathrm{U} / \mathrm{mL}$ and $40 \mathrm{U} / \mathrm{mL}$ there is a roughly $10 \%$ difference in the signal. As $35 \mathrm{U} / \mathrm{mL}$ is the cutoff for ovarian cancer diagnosis, the sensor is effective in differentiating between the two clinical states.

The nonspecific signal is less than half of the specific signal, but it does increase with the increase in CA-125 concentration. At $10 \mathrm{U} / \mathrm{mL}$, there is no positive change in impedance measured from the nonspecific electrodes. Even at $20 \mathrm{U} / \mathrm{mL}$ there is only a small change in impedance measured. The signal from the specific electrodes never reaches a saturation level implying that the sensor 
may be capable of detecting larger concentrations of CA-125. However, the nonspecific electrodes also do not produce a saturated signal implying that larger levels of nonspecific detection may be also be possible. More testing will be required to determine the upper boundary on the specific detection range and to determine how differentiable that signal is from the nonspecific signal generated at this detection boundary.

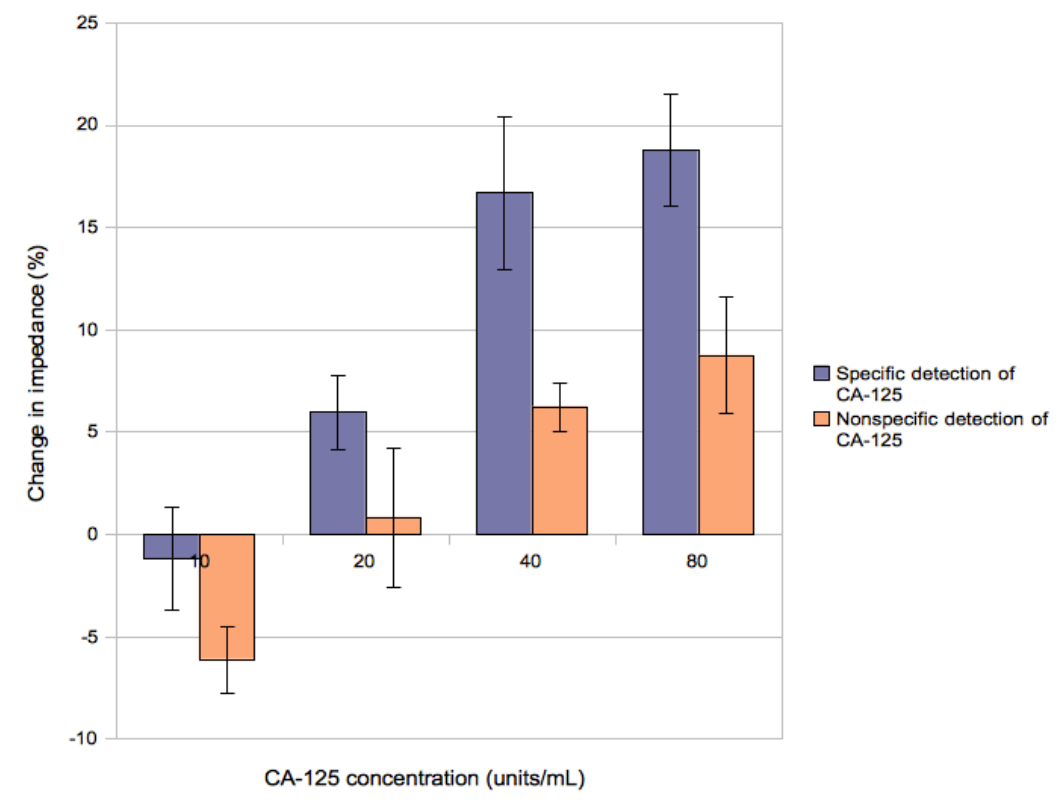

Fig 45. Multiplexed detection of CA-125. The data in purple is a result of the change in impedance that occurred when various concentrations of CA-125 in spiked buffer were detected by electrodes functionalized to specifically detect CA-125. The data in purple is the change in impedance that was measured when electrodes functionalized for the detection of CEA were exposed to various concentrations of CA-125 in spiked buffer. There is nonspecific detection measured for concentrations of CA-125 of 20 units $/ \mathrm{mL}$ and higher with the impedance change linearly correlated with the concentration of CA-125 present. All data collected at $500 \mathrm{~Hz}$. 
The multiplexed sensor was also used to detect CEA in spiked buffer. In the same way that nonspecific detection was measured for CA-125, when CEA at varying concentrations was exposed to the sensor EIS data was collected on electrodes functionalized to detect CEA and on the electrodes functionalized to detect CA-125. The impedance measurements collected on the electrodes specified for the detection of CA-125 generated the nonspecific data.

Figure 46 compares the change in impedance for specific and nonspecific detection of CEA at $500 \mathrm{~Hz}$. There is a linear increase in the change in impedance with increasing CEA concentration as shown in the purple data. The clinical range for diagnosis is above a concentration of $10 \mathrm{ng} / \mathrm{mL}$ and the sensor is capable of detecting CEA concentrations within this range. The change in impedance doesn't change by more than a few percentage points between 10 and $100 \mathrm{ng} / \mathrm{mL}$ implying that the signal is probably close to saturation. The nonspecific detection also increases with increasing concentration of CEA. Similar to the nonspecific detection of $\mathrm{CA}-125$, the nonspecific signal is roughly half of the specific signal making it differentiable from the specific signal. 


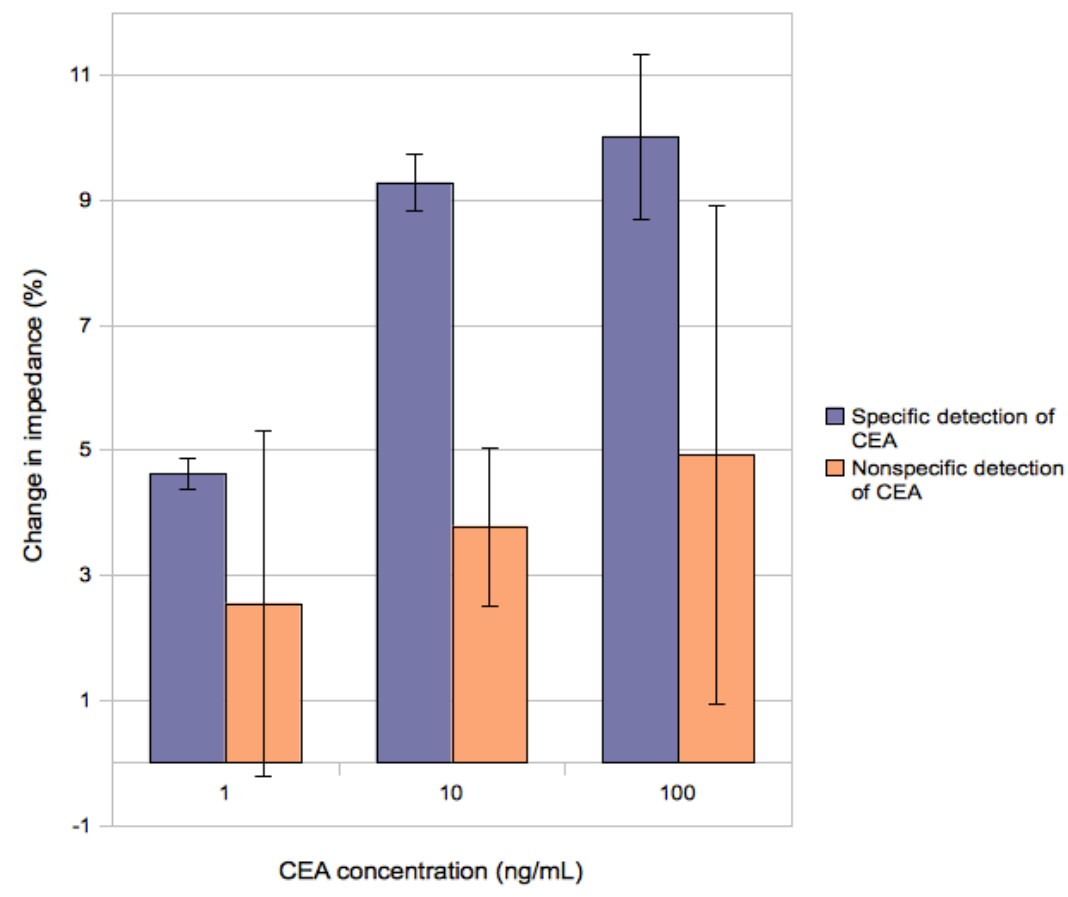

Fig 46. Multiplexed detection of CEA in spiked buffer. The data in purple represents the change in impedance measured when the electrodes functionalized for the detection of CEA were exposed to various concentrations of CEA. The data in orange is the change in impedance measured on electrodes that were functionalized for the detection of CA-125, not CEA. This data represents the nonspecific detection of CEA by the multiplexed biosensor. All data taken at $500 \mathrm{~Hz}$.

Figure 47 shows a side by side comparison of the specific and nonspecific detection of CA-125 and CEA in spiked buffer. The dark orange bars represent the change in impedance resulting from the specific detection of both markers and the light orange bars are the change in impedance from the nonspecific detection. The change in impedance is greater for CA-125 than it is for CEA which is the same trend seen in the sensors developed for individual detection of CA-125 and CEA. However, the signal from the nonspecific detection of CEA by the CA-125-functionalized electrodes is also larger than the signal from the nonspecific detection of CA-125 by the CEA. 
It seems there is a substantial nonspecific detection with the multiplexed sensor. One possible issue may be with the monoclonal antibodies used in the sensing layers. Both were obtained using human immunogens from different locations; the CEA from human liver and the CA-125 from human ascites fluid. It is also possible that the nonspecific binding sites were not completely blocked and that the nonspecific antigens bound to these sites. It is also possible that there is some cross reactivity that occurs between CA-125 and CEA. Both contain heavily glycosylated regions and there are only eight principal sugars in human glycoproteins. As such, there are likely some similar sugars between CA-125 and CEA which may lead to some overlap in observed binding.

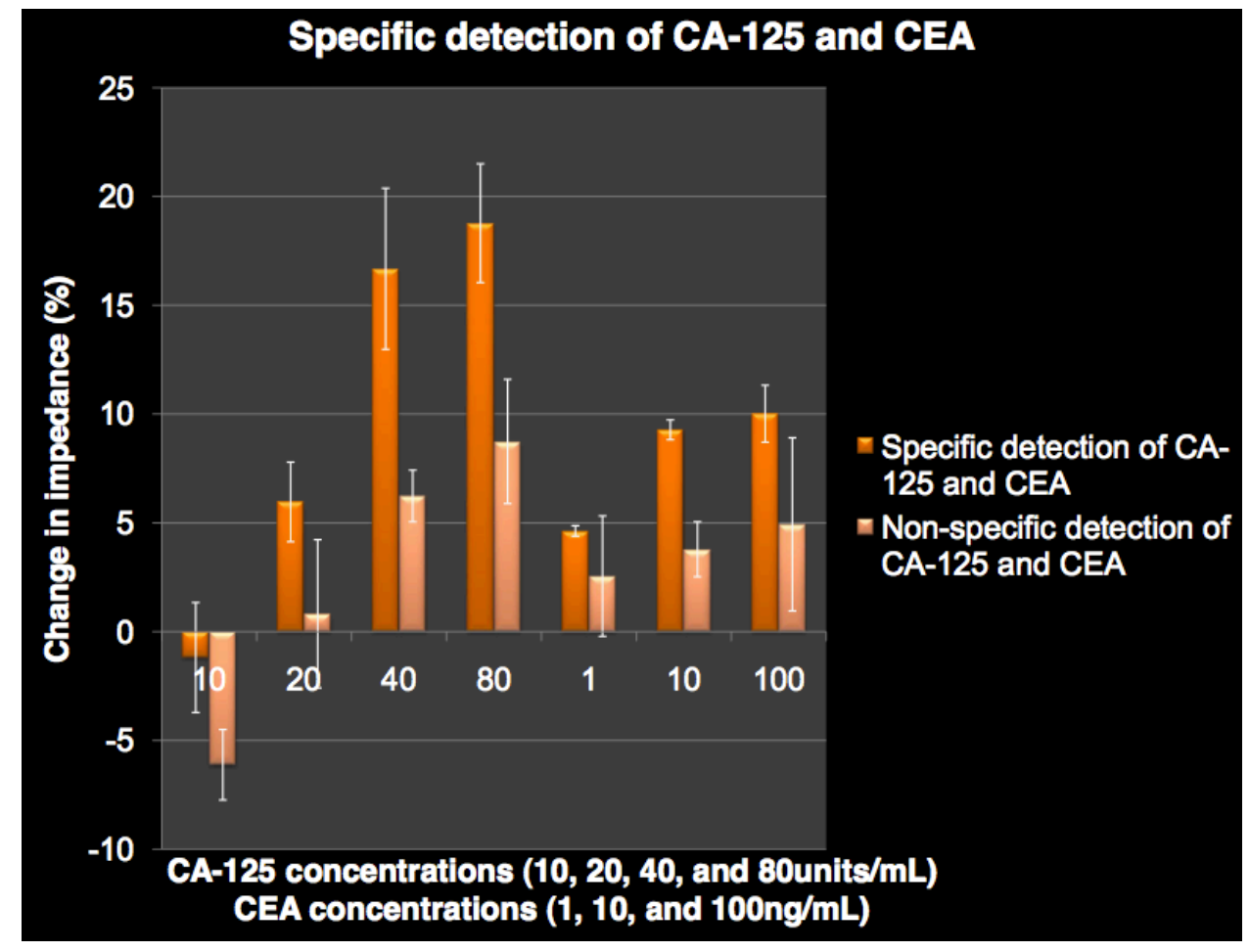

Fig 47. Direct comparison of multiplexed detection in spiked buffer. This is the same data as seen in Fig 43 and Fig 44 shown side by side. There is specific and nonspecific detection of CA-125 and CEA. The change in impedance is larger for the specific detection of CA-125 than for the detection of CEA, but the. All data collected at $500 \mathrm{~Hz}$. 
The multiplexed sensor was also used to detect CA-125 and CEA in spiked $5 \%$ rabbit serum. Figure 48 shows the direct comparison of specific and nonspecific detection for CA-125 and CEA over a narrow concentration range that falls within the range used for clinical diagnosis. Again, a differentiable signal was obtained at the diseased level for CA-125 and CEA, $40 \mathrm{U} / \mathrm{mL}$ and $10 \mathrm{ng} / \mathrm{mL}$, respectively. These signals are much larger than their spiked buffer counterparts. The change in impedance for CA-125 at $40 \mathrm{U} / \mathrm{mL}$ in buffer is about $19 \%$. In spiked serum it increases to $49 \%$. The signal from CEA does not experience as much augmentation. It goes from $10 \%$ in buffer for $100 \mathrm{ng} / \mathrm{mL}$ to $15 \%$ in serum for the same concentration. This would indicate that something in the serum besides CA-125 and CEA are binding to the sensing layer. Again, it may be a consequence of the antibodies used in the sensing layer. The specific signaling trends of the sensor when detecting the antigens in spiked buffer are also seen in the detection of the antigens in spiked $5 \%$ serum. For $\mathrm{CA}-125$, there is a large change in impedance between 20 and $40 \mathrm{U} / \mathrm{mL}$. For CEA, the change in impedance does not vary greatly between 10 and $100 \mathrm{ng} / \mathrm{mL}$.

The nonspecific detection of CA-125 by the CEA-specified electrodes does not experience much of an increase in the spiked serum sample. Even at $40 \mathrm{U} / \mathrm{mL}$, the nonspecific change in impedance remains below $10 \%$, just as it was in the spiked buffer. The nonspecific detection of CEA by the CA-125specified electrodes doesn't result in a positive change in impedance. Instead the impedance value drops when CEA is exposed to the sensing layer on the 
CA-125 specified electrodes. This may be a result of the shearing off of some material on the sensing layer that may occur while the chip is being washed. The result is that there is detection of $\mathrm{CA}-125$ and CEA in $5 \%$ rabbit serum within the clinical range though there is a question regarding what other serum proteins may be binding to the sensing layer and generating such a large signal, particularly for $\mathrm{CA}-125$. The nonspecific detection signals are minimal when compared to the specific detection signals.

Whether it is used for detection in spiked buffer or serum, it will be necessary to explore a fuller range of concentrations to determine the dynamic detection limits for this multiplexed biosensor. This is particularly true of the range for $\mathrm{CA}-125$ detection as even a $30-50 \%$ change in impedance, as seen in the data collected in $5 \%$ serum, does not saturate the signal. The signal from CEA seems to be close to saturation beyond $10 \mathrm{ng} / \mathrm{mL}$ and smaller concentrations remain to be tested. 


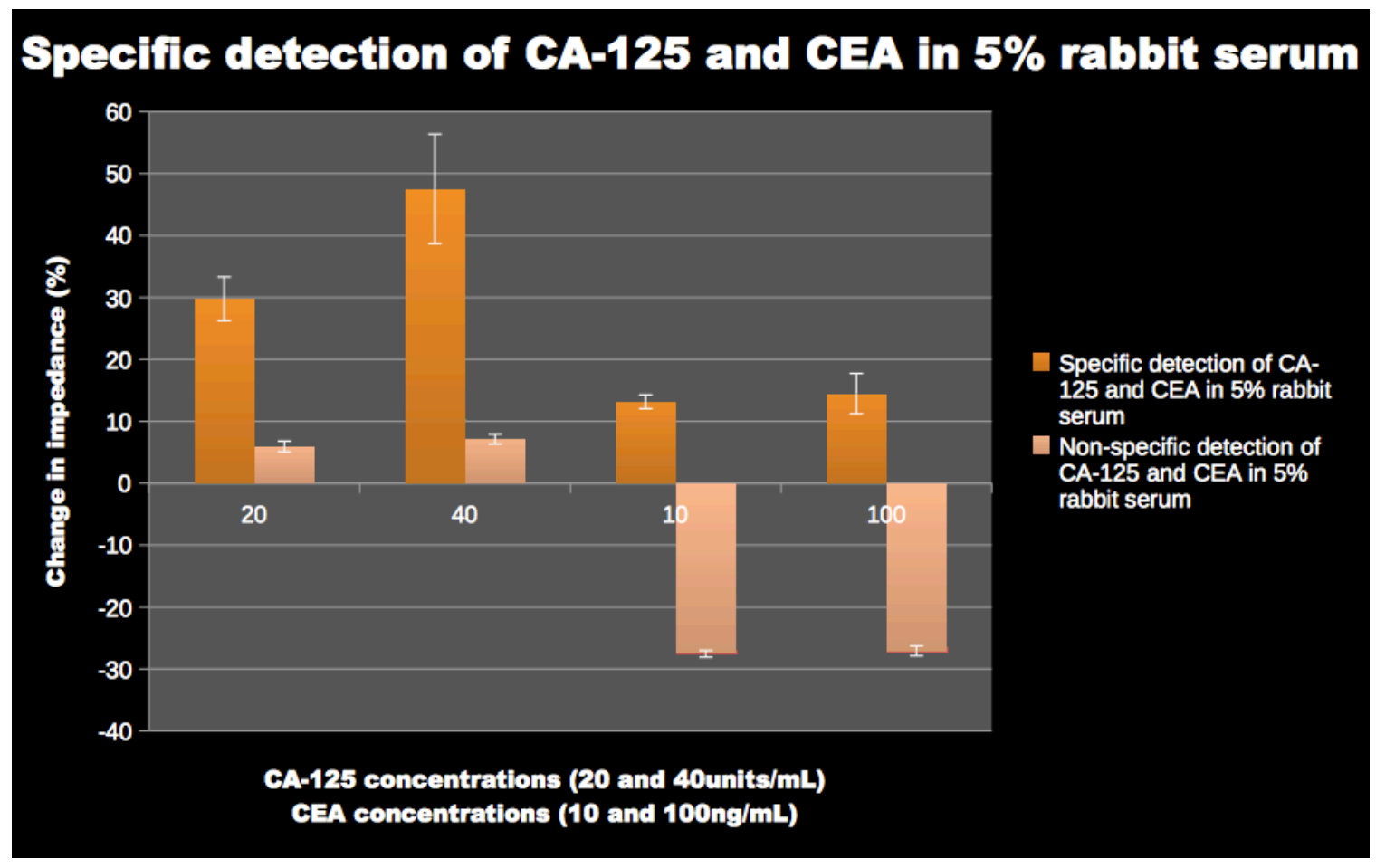

Fig 48. Direct comparison of detection of CA-125 and CEA in 5\% rabbit serum by the multiplexed chip. The dark orange bars represent the change in impedance resulting from the binding of CA-125 and CEA to the electrodes specified for their detection. The light orange bars are the change in impedance resulting from nonspecific detection of CA-125 and CEA by electrodes not specified for their detection. This nonspecific data for CA-125 was collected on the electrodes functionalized to detect CEA and the nonspecific data for CEA was collected on electrodes functionalized for the detection of CA-125. All data was collected at $500 \mathrm{~Hz}$.

Multiplexed sensors have been developed for the simultaneous detection of CEA and CA-125. A recently reported sensor used magnetic nanoparticles coated with antiCEA and antiCA-125 antibodies to generate a potential shift under manipulation by an external magnetic field [60]. This sensor contained five electrodes on a microfluidic device and all five were functionalized for the detection of a particular antigen. The target antigens besides CEA and CA-125 were alpha fetoprotein and CA 15-3. There was less than a 10\% change in signal created by the electrodes when they were exposed to proteins they were not 
functionalized to detect. CA-125 and CEA were detected at concentrations of 3.5, 30 , and $50 \mathrm{kU} / \mathrm{ml}$ and $2,50,100$, and $180 \mu \mathrm{g} / \mathrm{mL}$, respectively.

This nanoparticle based sensor offers several advantages. It is capable of detecting numerous targets simultaneously. It is capable of rapid detection; signals are generated between 30 s and 5 minutes. This is less than half of the time required for the EIS sensor described above to generate a signal. The cross reactivity is relatively low. It, like the EIS sensor described above, generates nonspecific signals below $10 \%$. However, this biomarker panel that is being detected is not specific to any one type of cancer but is instead a generic cancer panel. This could lead to an increased cost device cost owing to the development of the antibody-based sensing layers. The detection range is also unsatisfactory. The CA-125 and CEA concentrations detected were well above any used in a clinical situation. The multiplexed EIS sensor is a good step towards the ultimate development of a biosensor to detect the biomarker fingerprint of ovarian cancer.

\subsection{Results of aptamer development through sequence analysis and ELISA}

development. As previously stated, the aptamer selection process occurred in two stages. First, ten rounds of positive selection and six rounds of counter selection were carried out using the modified SELEX process. An ELISA was developed at that point to determine if the aptamer pool was suitable for molecular cloning. 
Figure 49 contains the results of that ELISA. The aptamer pool exhibits binding with CA-125 before and after the counter selection rounds with less binding evident at lower concentrations once the counter selection rounds have taken place. This implied that some sequences that preferred binding to BSA and CEA and had indeed been removed for the aptamer pool. The beginning of a concentration dependent curve was developed after the counter selection rounds. The binding between the aptamer pool and CEA was probed using ELISA. Binding between the pool and CEA both before and after the counter selection rounds was quantified. The binding between the pool and CEA did not appear to be diminished by the counter selection rounds. However, the signal generated by the aptamer pool and CA-125 binding was roughly twice the signal generated by the pool binding to CEA making it feasible for the aptamer pool to generate distinct signals when detecting the two different proteins. BSA, the other counter selective protein exhibited a small amount of binding with the aptamer pool both before and after the counter selection rounds implying that the aptamer pool did not show a propensity for binding to BSA to begin with.

Based on the ELISA data it was determined that the aptamer pool was suitable for molecular cloning. After cloning, using the process described in section 4.7, the plasmid DNA of 50 colonies were sequenced and 50 aptamer inserts were identified. Those aptamers were analyzed using the methods detailed in Chapter 5. From them five aptamer sequences were chosen for synthesis (Table 1). These five sequences were chosen for synthesis based on 
the quality of the sequencing data, the presence of certain nucleotide motifs, and structural similarities. One sequence was present in duplicate within the 50 sequenced colonies and it was automatically chosen for synthesis based on the idea that an aptamer that had high affinity for CA-125 would be present in larger numbers as it would have undergone several binding steps and multiple amplifications through PCR. In an ELISA exploring the binding capabilities of these synthesized sequences, none of these sequences exhibited any binding with CA-125. 


\section{ELISA for aptamer pools}

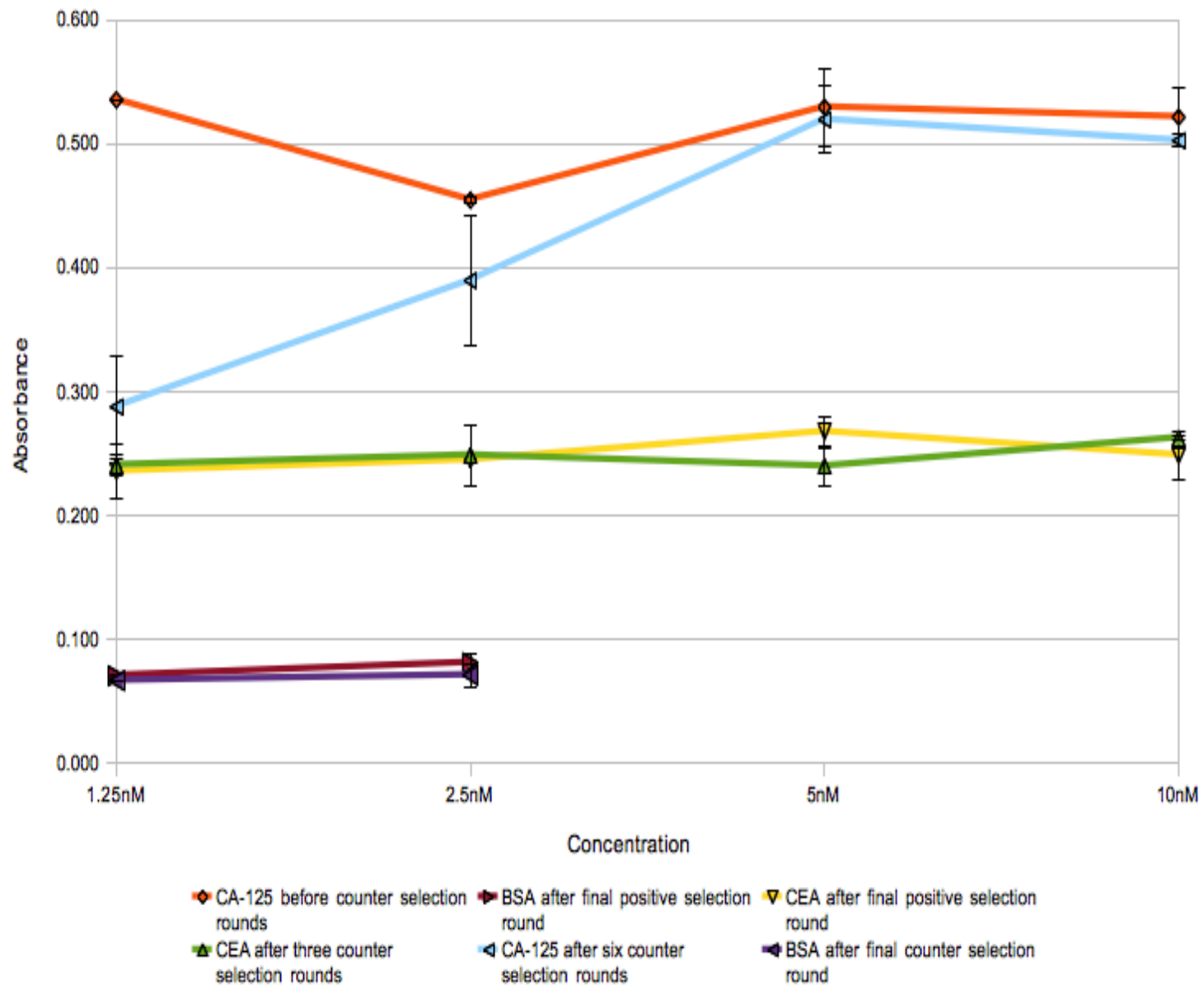

Fig 49. ELISA results from the initial aptamer pool after 16 modified SELEX rounds. The orange line is the binding between the aptamer pool and CA-125 after ten positive selection rounds. The yellow line is the binding between the aptamer pool and CEA after ten positive selection rounds and before any counter selection rounds. The blue line is the binding between the aptamer pool and CA-125 after six counter selection rounds on top of the ten positive selection rounds. There is less binding between the pool and CA-125 at lower concentrations than there is between the pool

and CA-125 before any counter selection has taken place indicating that some aptamer sequences were indeed removed from the pool during the six counter selection rounds. The green line is the binding between the aptamer pool and CEA after the six counter selection rounds have taken place. There is no discernible decrease in binding between the aptamer pool and CEA after these selection rounds. BSA exhibits little binding with the aptamer pool before or after the counter selection rounds. 


\begin{tabular}{|c|l|c|}
\hline $\begin{array}{c}\text { Sequen } \\
\text { ce ID }\end{array}$ & \multicolumn{1}{|c|}{ Aptamer Sequence } & $\begin{array}{c}\text { Number } \\
\text { of } \\
\text { copies }\end{array}$ \\
\hline 1 & $\begin{array}{l}\text { TAACCGTTAAGAGGACCACGGAATATATGGGCGAACGCGA } \\
\text { AACCTTGGACAGCTGACGCG }\end{array}$ & 2 \\
\hline 2 & $\begin{array}{l}\text { CTACAGCCAAGTGTGAGATCAAGATCGTAGAGAGTCGGCT } \\
\text { CTGGTCAGAGTATTGGGAGCG }\end{array}$ & 1 \\
\hline 3 & $\begin{array}{l}\text { AAATAGCGCGATTAAATGCAATGAACACTGAGAACTGTCC } \\
\text { GTAGACACGTTCTTACAATG }\end{array}$ & 1 \\
\hline 4 & $\begin{array}{l}\text { CTCAAACTGGGAGAGTGGAACATACAAGACATAACCGCTT } \\
\text { CAGAACTATGACACGTGTTA }\end{array}$ & 1 \\
\hline 5 & $\begin{array}{l}\text { ACGTGAGATCTGGTGCCGGAATACGCACTACTGACCAGAA } \\
\text { TCAAGCAAATACTAGAGTAT }\end{array}$ & 1 \\
\hline
\end{tabular}

Table 1. Summary of the five aptamer sequences chosen for synthesis. These aptamers had undergone ten positive selection rounds and six counter selection rounds, molecular cloning, and sequencing. Of the 50 aptamers chosen for sequencing, there was only one duplicate sequence.

The second stage of the aptamer selection process began after none of the synthesized aptamer exhibited any binding to CA-125. It was determined that the binding conditions may have been too lax to procure an aptamer pool with high affinity. To enrich the aptamer pool with highly selective aptamers, 18 additional positive selection rounds of the modified SELEX procedure were carried out. Nine of those rounds were done using Binding Buffer II and the remaining nine using Binding Buffer III. Each of these buffers contained a higher salt concentration than the binding buffer which preceded them, creating more stringent binding conditions for the aptamer pool. After 18 rounds an ELISA was used to determine if the additional positive selection rounds had enriched the aptamer pool and if the pool was suitable for molecular cloning. 
Figure 50 contains the results of that ELISA. The binding between the aptamer pool and CA-125 increases with the implementation of each successive binding buffer. There is more binding between CA-125 and the aptamer pool developed after 32 rounds of positive selection than the aptamer pool developed after ten rounds of positive selection. Knowing that the aptamer pool now exhibited more affinity for $\mathrm{CA}-125$ than it had when the initial molecular cloning procedure took place, it was decided that the pool was again suitable for molecular cloning. 


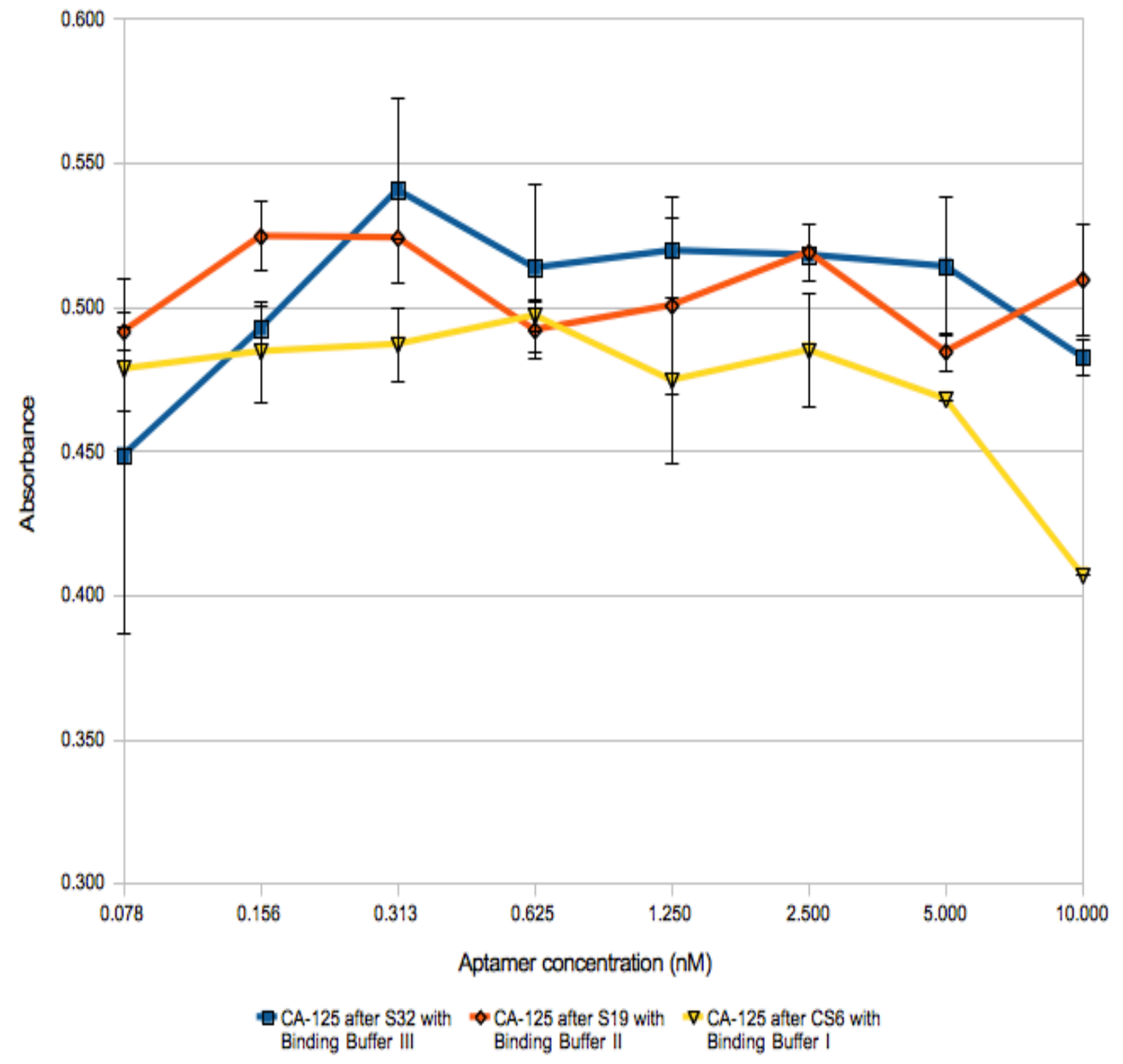

Fig 50. ELISA results from 18 additional rounds of modified SELEX. The yellow line is the binding between the aptamer pool and CA-125 using the initial binding buffer, Binding Buffer I. The orange line is the binding between the aptamer pool and CA-125 after nine additional rounds of positive selection using Binding Buffer II which contained a higher $\mathrm{NaCl}$ concentration than Binding Buffer I. The blue line is the binding between the aptamer pool and CA-125 after the final nine rounds of positive selection using Binding Buffer III which contained a higher concentration of $\mathrm{NaCl}$ and Tween 20 than either Binding Buffers I and II. The data indicates there is more binding between the aptamer pool and CA-125 under each successive binding buffer indicating that the aptamer pool has become more selective for CA-125.

After molecular cloning, 50 colonies were chosen and their plasmid DNA collected. This plasmid DNA was run through a PCR to amplify the aptamer insert. The individual aptamers were then isolated using gel electrophoresis. 
These 50 aptamers were then used in an ELISA to determine which of the aptamers would bind to CA-125 with the highest affinity and neglect to bind to CEA.

All 50 aptamers showed affinity for CA-125 and all 50 exhibited some binding with CEA. Table 2 contains a summary of the 14 aptamers that exhibited the highest affinity for CA-125 combined with the least affinity for CEA. These aptamers were then sequenced. Only aptamers that showed at least $60 \%$ more reactivity with CA-125 than CEA were selected and sequenced. 


\begin{tabular}{|l|l|}
\hline $\begin{array}{c}\text { Aptamer from Chosen } \\
\text { Colony }\end{array}$ & $\begin{array}{c}\text { \% More Activity with } \\
\text { CA-125 than CEA }\end{array}$ \\
\hline C4 & 62.04 \\
\hline C5 & 74.34 \\
\hline C6 & 63.59 \\
\hline C7 & 63.52 \\
\hline C9 & 65.78 \\
\hline C10 & 70.20 \\
\hline C15 & 65.07 \\
\hline C16 & 70.26 \\
\hline C17 & 62.55 \\
\hline C23 & 62.55 \\
\hline C25 & 77.91 \\
\hline C27 & 63.26 \\
\hline C30 & 63.96 \\
\hline C35 & 62.20 \\
\hline & \\
\hline
\end{tabular}

Table 2. Summary of aptamers after further refinement through an additional 18 positive selection rounds and molecular cloning. Fifty colonies were chosen after cloning and these colonies were boiled down and the plasmid DNA containing the aptamer insert was collected. The aptamer inserts were then amplified using PCR and used in an ELISA to determine which colonies had contained the aptamer inserts that exhibited the best binding with CA-125 and the ability to bind discriminately with CA-125 over CEA. All 50 colonies contained inserts that exhibited binding with CA-125 and the colonies listed above showed the strongest preference for binding with CA-125 over CEA.

The sequencing data from the 14 colonies was analyzed and it is summarized in Table 3. There were multiple copies of three of the sequences. Sequence 1 was identified four times and sequences 1 and 2 were each identified twice. The size of most of the sequences was unexpected. Whereas 
the expected size of the aptamer was $96 \mathrm{bp}$, most of the returned sequences were 76 bp long. There were two exceptions; sequences 8 and 9 were both 96 bp. These short sequences did bind to CA-125 during the ELISA so it can be assumed that the $20 \mathrm{nt}$ lost in the modified SELEX process were not key in the aptamer's binding ability. Figure 34 indicated that there were some PCR products that were not $96 \mathrm{bp}$, but it was a surprise to find so many shortened sequences among the few colonies we studied.

It is possible that these nucleotides were lost during the PCR process. It is not uncommon to lose nucleotides through PCR and it is assumed, being as the aptamer underwent 32 amplifications through PCR, that it is the culprit. Even just the occasional loss of a nucleotide in a single PCR could result in a large number of deletions over the course of so many PCRs. Regardless of the source of the deletions, the aptamers showed high binding and specificity for CA-125 making them suitable aptamers for CA-125. 


\begin{tabular}{|c|c|c|}
\hline $\begin{array}{l}\text { Sequence } \\
\text { ID }\end{array}$ & Aptamer Sequence & $\begin{array}{l}\text { Number of } \\
\text { Copies }\end{array}$ \\
\hline 1 & $\begin{array}{l}\text { TATCAATTACTTACCCTAGTGGTGTGATGTCGTA } \\
\text { TGGATG }\end{array}$ & 4 \\
\hline 2 & $\begin{array}{l}\text { AATGCCATGATTAGTATTCGTTGTGTGCAAGGT } \\
\text { GTT }\end{array}$ & 2 \\
\hline 3 & $\begin{array}{l}\text { ACCAGCGCTCGCGATAAGTTTTATTTATTTGTCA } \\
\text { TGTTCG }\end{array}$ & 2 \\
\hline 4 & $\begin{array}{l}\text { ACCGGATCATACACATTCTCACATGTAGTCGAC } \\
\text { ATTCTTT }\end{array}$ & 1 \\
\hline 5 & $\begin{array}{l}\text { TGTTAACTTATGGCGATGGGTTAGGGCTTTGTA } \\
\text { ATACTCG }\end{array}$ & 1 \\
\hline 6 & $\begin{array}{l}\text { TAATGGCCACTGGGACACAGCCTTCTCGTGTG } \\
\text { ACTAGGTT }\end{array}$ & 1 \\
\hline 7 & $\begin{array}{l}\text { TATCAATTACTTACCCTAGTGGTGTGATGTCGTG } \\
\text { TGGATG }\end{array}$ & 1 \\
\hline 8 & $\begin{array}{l}\text { ACATAAGGAGGGGCGTCAAGATGGGGGCAAG } \\
\text { GCAGAAGCAGAAAGTACAAAGGTAGGGTA }\end{array}$ & 1 \\
\hline 9 & $\begin{array}{l}\text { CGAGGATCAACAACGAGCAGCCATCCATCGAC } \\
\text { AAGTGCGAAACCAGCGTGCCACAATTGA }\end{array}$ & 1 \\
\hline
\end{tabular}

Table 3. Summary of the sequences of the nine aptamers identified after the second round of molecular cloning. After using an ELISA to determine the aptamers that exhibited the best binding to CA-125 and the least binding to CEA, the plasmid DNA was sequenced and the aptamer inserts identified. Multiple copies of three of the aptamers were found. Specifically, four copies of sequence 1 and 2 copies each of sequences 2 and 3 were identified. Multiple copies of the same aptamer indicate that the aptamer pool had been successfully enriched and become more selective based on the use of buffers creating more stringent binding conditions for the pool.

Secondary structures were generated for these 14 aptamers using mfold and compared to one another. Several conserved structures were identified 
among the 14 sequences. The results are in Figure 51. The boxed structures were found to be repeated in at least one other structure. There were also several other conserved structures not represented in Figure 51. Because the secondary structure of the aptamer is responsible for its binding ability with the target these repeating structures gave valuable information. They proved that the pool was enriched and that even though the sequences were different there were particular binding mechanisms being selected during the 32 modified SELEX rounds. There were two sequences of $96 \mathrm{bp}$ from the 14 that were sequenced. They did not have any structures in common. These two sequences did not have any common structures with the shortened sequences.

Analysis of these structures indicated that the 14 aptamers would all be suitable for synthesis and incorporation into a biosensor. There is more work that can be done to evaluate the aptamers which are discussed in section 7.3. 
a)

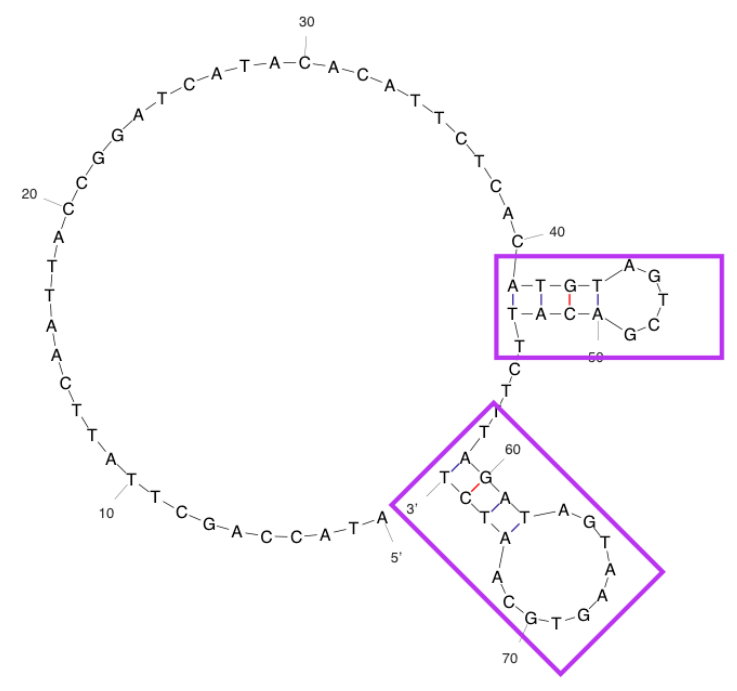

c)

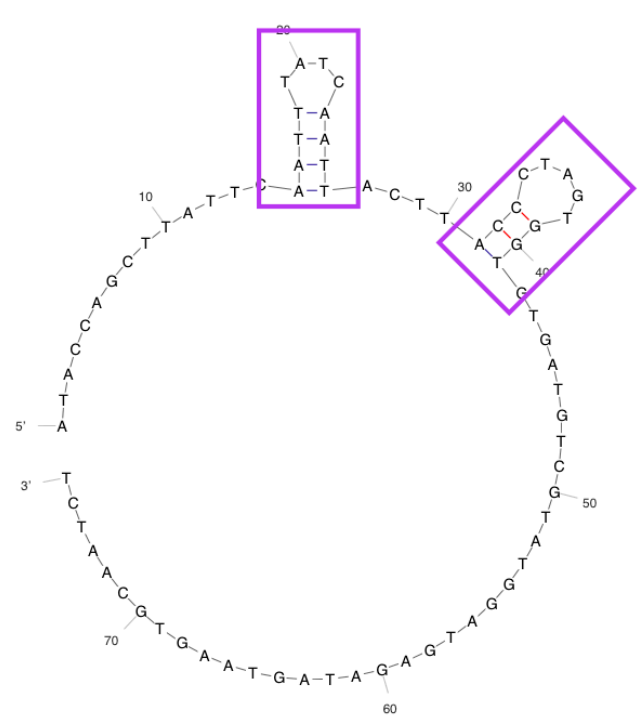

b)

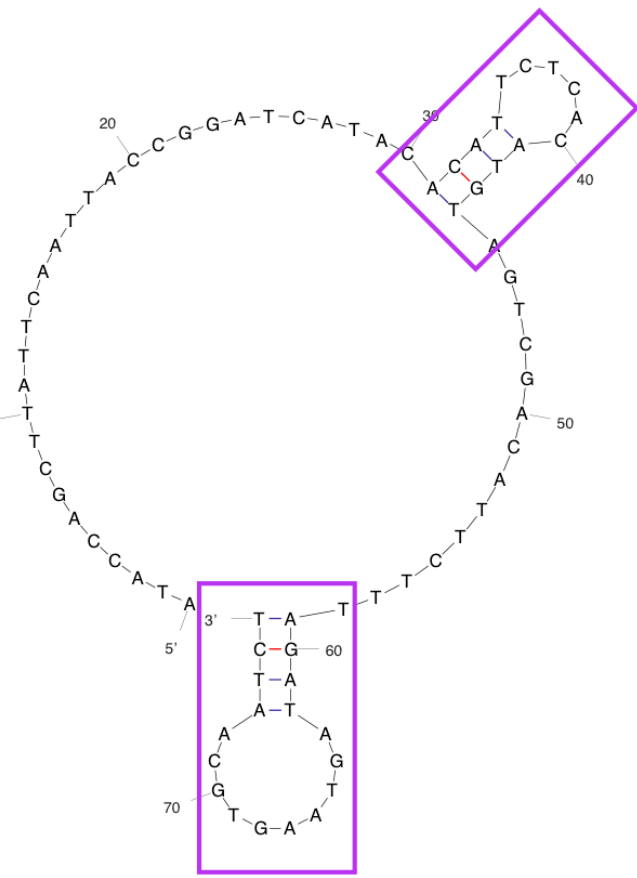

d)

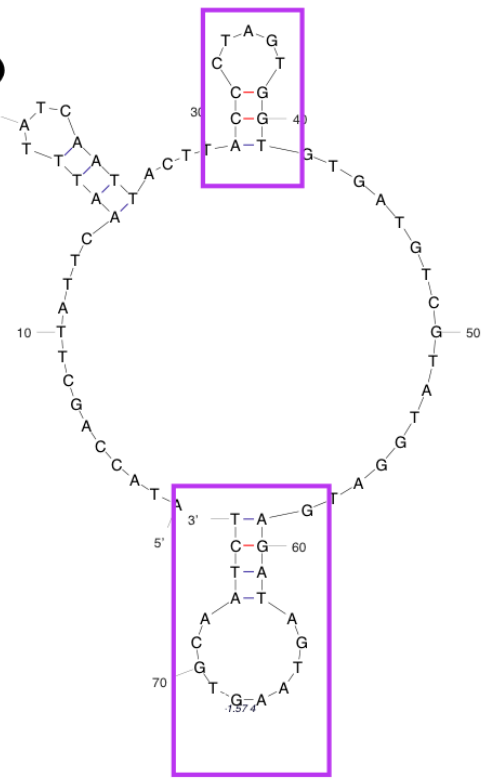

Fig 51. Common structures among the 14 selected aptamers. a) Structures repeated in four different sequences. b) Structures repeated in three different sequences. c) Structures repeated in two different sequences. d) Structures repeated in two different sequences. These repeated structures indicated that the aptamer pool had been enriched. The sequences were different but contained the same structures. 


\section{Chapter 7:}

Conclusions and suggestions for future work 


\subsection{Conclusions from CA-125 and CEA biosensor work. Biosensors to}

effectively detect CA-125 and CEA in spiked buffer were developed. The sensor to detect CA-125 produced a measurable signal at key concentrations of CA-125. When analyzing the overall change in impedance at $500 \mathrm{~Hz}$, the detection of CA-125 at a concentration of $10 \mathrm{U} / \mathrm{mL}$ produced a $16.13 \%$ change in impedance. At a CA-125 concentration of 80 units $/ \mathrm{mL}$, the impedance has increased to $24.15 \%$, resulting in an $8.02 \%$ change in impedance between the highest and lowest concentration of CA-125 detected by the biosensor. Most importantly, the biosensor was effective in differentiating between the clinical ranges for diseased and non-diseased diagnoses. The clinical limit is set at $35 \mathrm{U} / \mathrm{mL}$ with a concentration of CA-125 in serum higher than that indicating disease.

Future work with the CA-125 sensor should include further testing to determine the dynamic range of detection by the biosensor. Though not necessary in a clinical setting, the ability to detect CA-125 concentrations outside of the narrow diagnostic range could be beneficial in a research setting. The sensor should also be used to determine its effectiveness in detecting CA-125 in whole serum and whole blood samples.

Concentrations of CEA were effectively detected by a nonfaradaic EIS biosensor at $100 \mathrm{~Hz}$. At $10 \mathrm{pg} / \mathrm{mL}$, the lowest concentration tested, a 3.11\% change in impedance was measured and at $10 \mu \mathrm{g} / \mathrm{mL}$, the highest concentration detected, an $11.95 \%$ change in impedance was detected. The percentage change in impedance increases with every concentration up to a concentration of 
$10 \mathrm{ng} / \mathrm{mL}$. At $10 \mathrm{ng} / \mathrm{mL}$, a $12.07 \%$ change in impedance was measured and the signals from larger concentrations generated signals within $1.5 \%$ of that maximum.

Beyond $10 \mathrm{ng} / \mathrm{mL}$ the detection range failed to be dynamic. However, the biosensor was effective in differentiating between the clinical ranges for diseased and non-diseased diagnoses. The clinical limit is set at $10 \mathrm{ng} / \mathrm{mL}$ with a concentration of CEA in serum higher than that indicating disease. There is a $9 \%$ change in impedance between the lowest concentration detected and the concentration used to determine if disease is present.

Future work with the CEA sensor should include testing the sensor over a narrower range of concentrations. The concentrations detected by the biosensor range from $10 \mathrm{pg} / \mathrm{mL}$ to $10 \mu \mathrm{g} / \mathrm{mL}$ increasing each time by an order of magnitude. In a research setting it may be necessary to detect CEA at much finer concentration increments. The sensor should also be used to determine its effectiveness in detecting CEA in whole serum and whole blood samples.

Nonfaradaic sensors for CEA and CA-125 in 5\% rabbit serum were unsatisfactory. The data obtained for the detection of CEA was inconsistent with the data obtained for CEA detection in spiked buffer. Though the CA-125 sensor was capable of detecting CA-125 in spiked $5 \%$ rabbit serum in the clinical range for disease diagnosis, low concentrations outside of the diagnosis range were not detected as they were in spiked buffer. The shortcomings of these sensors in a 
complex sample like $5 \%$ rabbit serum provided the impetus for developing a better sensor through aptamer development.

The multiplexed sensor was successful in detecting CA-125 and CEA in both spiked buffer and 5\% rabbit serum. CA-125 was detected between 10 and $80 \mathrm{U} / \mathrm{mL}$ and CEA was detected between 1 and $100 \mathrm{ng} / \mathrm{mL}$. Both were detected at the key concentrations used for clinical diagnosis, between 20 and $40 \mathrm{U} / \mathrm{mL}$ for CA-125 and 1 and $10 \mathrm{ng} / \mathrm{mL}$ for CEA. Detection of CA-125 in spiked buffer at a concentration of $40 \mathrm{U} / \mathrm{mL}$ resulted in a $16.67 \%$ change in impedance and detection of CEA in spiked buffer at a concentration of $10 \mathrm{ng} / \mathrm{mL}$ resulted in a $9.28 \%$ change in impedance. The same concentrations in $5 \%$ rabbit serum generated a $48.26 \%$ and $15.46 \%$ change in impedance for CA-125 and CEA, respectively. The signals from the spiked serum are much larger than the signals from the spiked buffer indicating that some of the material present in the serum may be binding to the functionalized electrodes or nonspecific binding sites that were not blocked. However, the spiked serum concentrations for both CA-125 and CEA still generate increasing, differentiable signals for increasing concentrations and within the clinical range of diagnosis.

The nonspecific binding between CEA and the CA-125 specific electrodes and CA-125 and the CEA-specific electrodes was quantified. In both cases, the nonspecific signal was below a $10 \%$ change in impedance even for high concentrations of CEA and CA-125. This was true for the data in spiked buffer and spiked $5 \%$ rabbit serum. 
Future work with the multiplexed sensor should include functionalizing some of the electrodes to detect $\mathrm{He} 4$ to create a sensor to detect all the ovarian cancer biomarkers. A larger range of concentrations in diluted serum will be necessary to determine the dynamic range. The multiplexed sensor should also be tested in whole serum and blood samples.

\subsection{Conclusions from and future work with the He4 biosensor. He4 was} detected using a labeled, faradaic EIS biosensor. The range used for clinical diagnosis falls between 3 and $10 \mathrm{ng} / \mathrm{mL}$ and He 4 was clearly detected both within and outside of this range. Differentiable $\Delta R_{C T}$ values were obtained from Nyquist plots for He4 concentrations between 1.56 and $100 \mathrm{ng} / \mathrm{mL}$ in spiked buffer. These $\Delta R_{C T}$ values were obtained for every labeled layer formed on the electrodes. The values obtained from just the first labeled layer generated sensitive, differentiable signals. An He4 concentration of $3.125 \mathrm{ng} / \mathrm{mL}$ generated a $\Delta \mathrm{R}_{\text {ст }}$ of about $250 \Omega$ and an He4 concentration of $12.5 \mathrm{ng} / \mathrm{mL}$ generated a $\Delta R_{\mathrm{CT}}$ of $1500 \Omega$. Thus, differentiable signals within the diagnosis range were generated using just the biotinylated antibodies. The subsequent layers of streptavidin-HRP and TMB precipitate increased those $\Delta R_{C T}$ values.

Detection below and beyond the clinical range was achieved. With just the biotinylated layer an $\mathrm{He} 4$ concentration of $1.56 \mathrm{ng} / \mathrm{mL}$ generated a $\Delta R_{\mathrm{CT}}$ value of around $125 \Omega$ and an He4 concentration of $25 \mathrm{ng} / \mathrm{mL}$ generates a $\Delta R_{C T}$ value of $3250 \Omega$. A signal is generated at up to $100 \mathrm{ng} / \mathrm{mL}$, but it is significantly smaller 
than the signal for $25 \mathrm{ng} / \mathrm{mL}$ implying that the signal is saturated beyond that concentration. All the $\Delta \mathrm{R}_{\mathrm{CT}}$ values mentioned above were amplified with the formation of every labeled layer and the same trend of increasing $\Delta R_{\mathrm{CT}}$ values for every concentration of $\mathrm{He} 4$ was observed. For the sake of simplicity only the values obtained with the biotinylated layer are mentioned.

Future work will include testing patient serum samples with the sensor to determine the presence of $\mathrm{He} 4$. These samples are currently being obtained from OHSU and testing will begin in the near future.

\subsection{Conclusions from developing an aptamer for CA-125 and future}

development of aptamers. Nine possible aptamers for CA-125 have been identified after 32 rounds of a modified SELEX procedure. Three of those sequences hold particular promise as multiple duplicate copies of them were identified in a relatively small pool of molecular clones.

Further work will include determining the most viable aptamer among the nine possible sequences. The goal is to develop a biosensor for CA-125 that replaces the monoclonal antibody currently used in the biosensors with one of the selected aptamers. Binding assays will be required to determine the aptamer with the highest binding affinity for $\mathrm{CA}-125$. The determination of the dissociation constant, $\mathrm{K}_{\mathrm{D}}$, for each aptamer from the binding assays will play a large role in determining the best aptamer. Competitive binding assays will also be needed to chose an aptamer. The competitive binding assay should compare the binding 
ability between the aptamer and CA-125 even when a monoclonal CA-125 antibody is present and presumably competing with the aptamer for the available binding sites. ELISA will also be an important assay tool in determining the most suitable aptamer. Through ELISA an aptamer can be selected that will bind to CA-125 under possible working conditions for the biosensor, i.e. higher temperatures, specialized buffers.

The combination of these different assays will yield the most suitable aptamer and it will be incorporated into a biosensor. This will ideally produce a cheaper sensor capable of more sensitive and more specific detection than the antibody based biosensor we have developed. 


\section{References}

[1] Gynecologic Neoplasms Merck Manual of Diagnosis and Therapy Professional Edition.

[2] Goff BA, Mandel L, Muntz HG, and Melancon CH. 2000. Ovarian cancer diagnosis. Cancer 89(10): 2068-2075.

[3] Survival rates based on SEER incidence and NCHS mortality statistics, as cited by the National Cancer Institute.

[4] General information about ovarian epthelial cancer, as cited by the National Cancer Institute.

[5] Gold P, Freedman SO. 1965. Carcinoembryonic antigen in faeces. J. Exp. Med. 121: 439-462.

[6] Tumor markers, as cited by the American Cancer Society.

[7] Bast RC Jr., Feeney M, Lazarus H, Nadler LM, Colvin RB, and Knapp RC. 1981. Reactivity of a monoclonal antibody with human ovarian carcinoma. J. Clin. Invest. 68(5): 1331-1337.

[8] Yin BW, Lloyd KO. 2001. Molecular cloning of the CA-125 ovarian cancer antigen. J. Biol. Chem. 29(20): 27371-27375.

[9] Gold P, Freedman SO. 1965. Specific carcinoembryonic antigens of the human digestive system. J. Exp. Med. 122: 467-481.

[10] Hellstrom I, et al. 2003. The He4 (WFDC2) protein is a biomarker for ovarian cancer. Cancer Res 63(13): 3695-700. (2003).

[11] Fujirebio Diagnostics. 2011. Product insert for prod. no. 404-10US. He4 EIA. [12] Bingle L, Singleton V, Bingle CD. 2002. The putative ovarian tumour marker gene HE4 (WFDC2), is expressed in normal tissues and undergoes complex alternative splicing to yield multiple protein isoforms. Oncogene 21: 2768-2773. [13] Ranganathan S, Simpson KJ, Shaw DC, Nicholas KR. 1999. The whey acidic protein family: a new signature motif and three-dimensional structure by comparative modeling. J Mol Graph Model. 17: 350-357.

[14] Manz A, Pamme N, and lossifidis D. Bioanalytical Chemistry. Imperial College Press (London, England) (2004).

[15] Clark LC Jr. 1956. Monitor and control of blood and tissue oxygen tensions. Jnr. Trans. Am. Soc. Artif. Intern. Organs 2: 41-48.

[16] Clark LC Jr., Lyons, C. 1962. Electrode systems for continuous monitoring in cardiovascular surgery. Jnr. Ann. NY Acad. Sci. 102: 29-45.

[17] lanniello RM, Lindsay TJ, and Yacynych AM. 1982. Differential pulse voltametric study of direct electron transfer in glucose oxidase chemically modified graphite electrodes. Anal. Chem. 54: 1098-1101.

[18] Ghindilis AL, Kurochkin IN. 1994. Glucose potentiometric electrodes based on mediatorless bioelectric catalysis. A new approach. Biosens. Bioelectron. 9: 353-359.

[19] Bartlett PN, Tebbutt P, and Whitaker RC. 1991. Kinetic aspects of the use of modified electrodes and mediators in bioelectrochemistry. Prog. React. Kinet. 16: 55-155. 
[20] Bosch ME, Sanchez A, Rojas F, and Ojeda C. 2007. Recent development in optical fiber biosensors. Sensors 7: 797-859.

[21] Liedberg B, Nylander C, and Lundstrm I. 1983. Surface plasmon resonance for gas detection and biosensing. Sensors and Actuators 4: 299-304.

[22] Lion-Dagan M, Ben-Dov I, and Willner I. 1997.Microgravimetric quartzcrystal microbalance analysis of cytochrome $c$ interactions with pyridine and pyridine-nitrospiropyran monolayer electrodes: characterization of inter-protein complexes at the functionalized surfacesColloids Surf. B: Biointerfaces 8 : 251-260.

[23] Ben-Dov I, Willner I, and Zisman E. 1997. Piezoelectric immunosensors for urine specimens of Chlamydia trachomatis employing quartz crystal microbalance microgravimetric analyses. Anal. Chem. 69: 3506-3512. [24] Bardea A, Dagan A, Ben-Dov I, Amit B, and Willner I. 1998. Amplified Microgravimetric Quartz-Crystal-Microbalance Analyses of Oligonucleotide Complexes: A Route to a Tay-Sachs Biosensor Device. Cem. Commun. 839-840. [25] O'Sullivan CK, Vaughan R, and Guibalt GG. 1999. Piezoelectric immunosensors- theory and applications. Anal. Lett. 32: 2353-2377. [26] Bard AJ, Faulkner LR. Electrochemical Methods: Fundamentals and Applications. Wiley (New York) (1980).

[27] Stoynov ZB, Grafov BM, Savova-Stoynova BS, and Elkin VV. Electrochemical Impedance. Nauka (Moscow) (1991). [28] Randles JEB. 1947. Kinetics of rapid electrode reactions. Discuss Faraday Soc. 1: 11-19.

[29] Ershler BV. 1947. Investigation of electrode reactions by the method of charging curves and with the aid of alternating currents. Discuss Faraday Soc. 1: 269.

[30] Athey D, Ball M, McNeil CJ, Armstrong RD. 1995. A study of enzyme catalyzed product deposition on planar gold electrodes using electrical impedance measurement. Electroanalysis 7(3): 270-273.

[31] Nahir TM, Bowden EF. 1996. The distribution of standard rate constants for electron transfer between thiol-modified electrodes and adsorbed cytochrome-c. Electroanal. Chem. 410: 9-13.

[32] Katz E, Willner I. 2003.Probing biomolecular interactions at conductive and semiconductive surfaces by impedance spectroscopy: routes to impedimetric immunosensors, DNA sensors, and enzyme biosensors. Electroanalysis 15: 913-947.

[33] Bresler HS, et al. 1992. Biosensor Design and Applications, ACS Symposium 511: 89-104.

[34] Newman AL, Hunter KW, Stanbro WD. 1986. The capacitive affinity sensor: a new biosensor. Chemical Sensors: 2nd International Meeting, Proc. 596-598. [35] Taylor RF, Marenchic IG, Cook EJ. 1988. An acetylcholine receptor-based biosensor for the detection of cholinergic agents. Anal. Chim. Acta 213: 131-138. [36] Taylor RF, Marenchic IG, Spencer RH. 1991. Antibody- and receptor-based biosensors for detection and process control. Anal. Chim. Acta 249: 67-60. 
[37] Mirsky VM, Riepl M, Wolfbeis OS. 1997. Capacitive monitoring of protein immobilization and antigen-antibody reaction on the mono-molecular films of alkylthiols adsorbed on gold electrodes. Biosens. Bioelectron. 12: 997-989. [38] Rickert J, Gopel W Beck W, Jung G, Heiduschka P. 1996. A 'mixed' selfassembled monolayer for an impedimetric immunosensor. Biosens. Bioelectron. 11(8): 757-768.

[39] Ameur S, Martelet C, Jaffrezie-Renault N, Chovelon JM. 2000. Sensitive immunodetection through impedance measurements onto gold functionalized electrodes. Appl. Biochem. Biotechnol. 89: 161-170.

[40] Zhenzhen L, Xiaohong L, Heinz-Bernhard K. 2011 Impedimetric Immobilized DNA-Based Sensor for Simultaneous Detection of $\mathrm{Pb}^{2+}, \mathrm{Ag}^{+}$, and $\mathrm{Hg}^{2+}$. Anal. Chem. 83(17): 6896-6901.

[41] Hassen WH, Chaix C, Abdelghani A, Beussueille F, Leonard D, JaffrezieRenault N. 2008. Sensors and Actuators B: Chemical 134(2): 755-760. [42] Park J, Lee Y, Chang B, Kim BH, Jeon S, Park S. 2010. Label-Free Impedimetric Sensor for a Ribonucleic Acid Oligomer Specific to Hepatitis C Virus at a Self-Assembled Monolayer-Covered Electrode. Anal. Chem. 82(19): 83428348.

[43] Pandey CM, Sumana G, Malhotra BD. 2011. Microstructured Cystine Dendrites-Based Impedimetric Sensor for Nucleic Acid Detection.

Biomacromolecules 12(8): 2925-2932.

[44] Chen W, Lu Z, Li CM. 2008.Sensitive Human Interleukin 5 Impedimetric Sensor Based on Polypyrrole-Pyrrolepropylic Acid-Gold Nanocomposite. Anal. Chem. 80(22): 8485-8492.

[45] Tuerk C, Gold L. 1990. Systematic evolution of ligands by exponential enrichment: RNA ligands to bacteriophage T4 DNA polymerase. Science 249: 505-510.

[46] Ellington AD, Szostak JW. 1990. In vitro selection of RNA molecules that bind specific ligands. Nature 346: 818-822.

[47] Hermann T, Patel DJ. 2000. Adaptive recognition by nucleic acid aptamers. Science 287: 820-825.

[48] Van Simaeys D, López-Colón D, Sefah K, Sutphen R, Jimenez E, et al. 2010. Study of the molecular recognition of aptamers selected through ovarian cancer cell-SELEX PLoS ONE 5(11). [49] Archemix website. 2011. www.archemix.com.

[50] Jenison R, Gill SC, Pardi A, Polisky B. 1994. High-resolution molecular discrimination by RNA. Science 263: 1425-1429.

[51] Tombelli S, Minunni M, Mascini M. 2005. Analytical applications of aptamers. Biosens. Bioelectron. 20: 2424-2434.

[52] Laitinen OH, Hytonen VP, Nordlund HR, Kulomaa MS. 2006. Genetically engineered avidins and streptavidins. Cell. Mol. Life Sci. 63(24): 2992-3017. [53] Stoltenburg R, Reinemann C, and Strehlitz B. 2005. FluMag SELEX as an advantageous method for selecting DNA aptamers. Anal Bioanal Chem 383: 8391. 
[54] Neduva V. et al. 2005. Systematic discovery of peptides mediating protein interaction network. PLoS Biology 3: 405.

[55] Zuker M. 2003. Mfold web server for nucleic acid folding and hybridization prediction. Nucleic Acids Res. 31(13): 3406-3415.

[56] Tang D, Su B, Tang J, Ren J, Chen G. 2010. Nanoparticle-Based Sandwich Electrochemical Immunoassay for Carbohydrate Antigen 125 with Signal Enhancement Using Enzyme-Coated Nanometer-Sized Enzyme-Doped Silica Beads. Anal. Chem. 82: 1527-1534.

[57] Das J, Kelley S. 2011. Protein Detection Using Arrayed Microsensor Chips: Tuning Sensor Footprint to Achieve Ultrasensitive Readout of CA-125 in Serum and Whole Blood. Anal. Chem. 83(4): 1167-1172.

[58] Altintus Z, Uludag Y, Gurbuz Y, Tothill IE. 2011. Surface plasmon resonance based immunosensor for the detection of the cancer biomarker carcinoembryonic antigen. Talanta 86: 377-383.

[59] Kozak D, Chen A, Marcon L, Trau M. 2008. Optically Encoded Particles as a High-Throughput Biosensor Platform. Talk at Trends in Nanotechnology Conference.

[60] Tang D, Yuan R, Chai Y. 2007. Magnetic control of an electrochemical microfluidic device with an arrayed immunosensor for simultaneous multiple immunoassays. Clin. Chem. 53(7): 1323-1330. 